The Astrophysical Journal Supplement Series, 164:450-476, 2006 June

(C) 2006. The American Astronomical Society. All rights reserved. Printed in U.S.A.

\title{
A 2 MILLIMETER SPECTRAL LINE SURVEY OF THE STARBURST GALAXY NGC 253
}

\author{
S. Martín and R. Mauersberger \\ Instituto de Radioastronomía Milimétrica (IRAM), Avda. Divina Pastora 7, Local 20, E-18012 Granada, Spain; martin@iram.es \\ J. Martín-Pintado \\ Departamento de Astrofisíca Molecular e Infrarroja, Instituto de Estructura de la Materia, CSIC, Serrano 121, E-28006 Madrid, Spain \\ C. HenKel \\ Max-Planck-Institut für Radioastronomie, Auf dem Hügel 69, D-53121 Bonn, Germany \\ AND \\ S. García-Burillo \\ Observatorio de Madrid, Alfonso XII, 3, 28014 Madrid, Spain \\ Received 2005 November 28; accepted 2006 February 8
}

\begin{abstract}
We present the first unbiased molecular line survey toward an extragalactic source, namely the nuclear region of the starburst galaxy NGC 253. The scan covers the frequency band from 129.1 to $175.2 \mathrm{GHz}$, i.e., most of the $2 \mathrm{~mm}$ atmospheric window. We identify 111 spectral features as transitions from 25 different molecular species. Eight of which (three tentatively) are detected for the first time in the extragalactic interstellar medium. Among these newly detected species, we detected the rare isotopomers ${ }^{34} \mathrm{SO}$ and $\mathrm{HC}^{18} \mathrm{O}^{+}$. Tentative detections of two deuterated species, DNC and $\mathrm{N}_{2} \mathrm{D}^{+}$, are reported for the first time from a target beyond the Magellanic Clouds. In addition, three hydrogen recombination lines are identified, while no organic molecules larger than methanol are detected. Column densities and rotation temperatures are calculated for all the species, including an upper limit to the ethanol abundance. A comparison of the chemical composition of the nuclear environment of NGC 253 with those of selected nearby galaxies demonstrates the chemical resemblance of IC 342 and NGC 4945 to that of NGC 253. On the other hand, the chemistries characterizing NGC 253 and M82 are clearly different. We also present a comparison of the chemical composition of NGC 253 with those observed in Galactic prototypical sources. The chemistry of NGC 253 shows a striking similarity with the chemistry observed toward the Galactic center molecular clouds, which are thought to be dominated by low-velocity shocks. This resemblance strongly suggests that the heating in the nuclear environment of NGC 253 is dominated by the same mechanism as that in the central region of the Milky Way.
\end{abstract}

Subject headings: galaxies: abundances — galaxies: individual (NGC 253) — galaxies: ISM — galaxies: nuclei — galaxies: starburst — radio lines: galaxies — surveys

\section{INTRODUCTION}

Our knowledge of the chemical composition of the interstellar medium (ISM) in the nuclei of external galaxies has so far been restricted to only $\sim 30$ molecular species (see $\S 4.1$ ), which is still far from the 129 molecules detected in the interstellar and circumstellar medium within our Galaxy (Lovas 2004). The initial detection of most of these 129 molecules has been the result of unbiased frequency scans toward specific Galactic sources, such as massive star-forming regions (e.g., Sgr B2(M), Cummins et al. 1986; OriMC-1, Lee et al. 2001), cold molecular clouds (e.g., TMC-1, Kaifu et al. 2004), and evolved stars (e.g., IRC +10216, Cernicharo et al. 2000). Spectral line scans toward Galactic sources focused mainly on the hot cores associated with massive starforming regions such as Sgr B2 and Orion-KL due to their complex chemistry as well as the brightness of their lines. Extragalactic molecular spectroscopy has been so far limited to selecting the strongest features seen in the Galactic center and disk sources and observing them toward well selected extragalactic targets. The most ambitious census of the molecular content of an external galaxy is the compilation of data toward the starburst galaxy NGC 4945 by Wang et al. (2004). Their multiline study combines observations of 19 molecular species previously known in other extragalactic sources.

Up to now unbiased molecular line surveys of extragalactic sources do not exist in spite of providing a powerful tool for de- termining the physical parameters of the molecular ISM. Observations of a large number of molecular lines with similar angular resolution allow us to describe the chemical complexity of the source, which provides fundamental information on the physical processes heating the medium in heavily obscured regions. In addition, frequency scans lead to serendipitous detections of new species.

The nuclear starburst of the galaxy NGC 253, the target of this survey, is one of the brightest and most prolific (in terms of molecular line detections) extragalactic molecular line sources. The Sculptor galaxy NGC 253 is an almost edge-on barred spiral $\left(i=72^{\circ}-78^{\circ}\right.$, Puche et al. 1991; Pence 1981) classified as type $\mathrm{SAB}(\mathrm{s}) \mathrm{c}$ (de Vaucouleurs et al. 1991) or Sc(s) (Sandage \& Tammann 1987). At a distance of $\sim 3 \mathrm{Mpc}$ (e.g., Mouhcine et al. 2005), NGC 253 is one of the nearest archetypes of nuclear starburst galaxies (Rieke et al. 1980). Its nuclear region contains one of the brightest extragalactic $I R A S$ sources with a $100 \mu \mathrm{m}$ flux of $1860 \mathrm{Jy}$ (Soifer et al. 1989). In fact, most of the overall IR luminosity of this galaxy $\left(L_{\mathrm{IR}}=2.1 \times 10^{10} L_{\odot}, \mathrm{SFR}_{\mathrm{IR}}=3.6 M_{\odot} \mathrm{yr}^{-1}\right.$, Strickland et al. 2004) stems from the regions of intense massive star formation within its central few hundred parsecs. Violent massive star formation is also revealed by the high supernova rate of $0.05-0.3 \mathrm{yr}^{-1}$ in the nuclear region of this galaxy (Mattila \& Meikle 2001; Ulvestad \& Antonucci 1997).

The high nuclear star formation activity is driven by huge amounts of molecular gas in the central few hundred parsecs of 


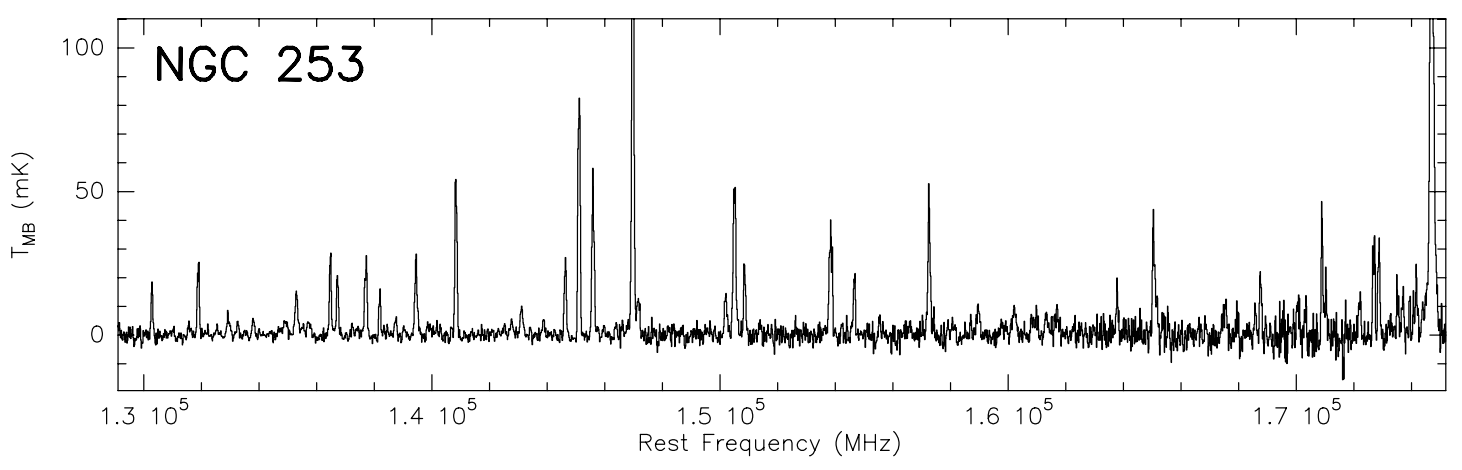

FIG. 1.-Complete $2 \mathrm{~mm}$ spectral frequency scan toward the nuclear region of NGC 253.

NGC $253\left[(1.3-2.6) \times 10^{9} M_{\odot} ;\right.$ Canzian et al. 1988; Mauersberger et al. 1996], likely powered by the bar structure first detected at near-infrared wavelengths (Scoville et al. 1985; Forbes \& Depoy 1992). Position-velocity diagrams from high-resolution observations of the nuclear region of NGC 253 show that the molecular material seems to follow orbital motions around the dynamical center, which are interpreted as $x_{1}$ and $x_{2}$ orbits within the context of a barlike potential (OH, Turner, 1985; CO, Canzian et al. 1988; Das et al. 2001; Paglione et al. 2004; HCN, Paglione et al. 1995; CS, Peng et al. 1996; $\mathrm{SiO}, \mathrm{H}^{13} \mathrm{CO}^{+}$, García-Burillo et al. 2000).

Here we present the first unbiased molecular line survey carried out toward a source outside the Milky Way. Technically, this survey was driven by the availability of two new wide-band $(1 \mathrm{GHz})$ receivers at $2 \mathrm{~mm}$ and new $1 \mathrm{GHz}$ wide filter bank spectrometers, together with the large collecting area of the IRAM $30 \mathrm{~m}$ telescope. Species such as $\mathrm{NO}, \mathrm{NS}, \mathrm{SO}_{2}, \mathrm{H}_{2} \mathrm{~S}$ and $\mathrm{H}_{2} \mathrm{CS}$, detected for the first time toward a starburst environment, were previously reported by Martín et al. (2003, 2005). Additional new molecules together with a full analysis of the data are presented.

\section{OBSERVATIONS AND RESULTS}

The first molecular frequency scan of an external galaxy was carried out at $2 \mathrm{~mm}$ with the $30 \mathrm{~m}$ IRAM telescope at Pico Veleta, Spain, between 2001 and 2004. It covers $\sim 86 \%$ of the observable $2 \mathrm{~mm}$ atmospheric window, from 129.1 to $175.2 \mathrm{GHz}$ (Fig. 1). At these frequencies, the telescope beamwidth ranges from $19^{\prime \prime}$ to $14^{\prime \prime}$. Figure 2 shows the size of the $30 \mathrm{~m}$ beam on top of an interferometric map of $\mathrm{SiO}$ emission (García-Burillo et al. 2000). A $K$-band image of NGC 253 (Engelbracht et al. 1998) is displayed in gray scale to illustrate the size of the observed region relative to the galaxy.

The observations were pointed at the position $\alpha_{\mathrm{J} 2000}=$ $00^{\mathrm{h}} 47^{\mathrm{m}} 33^{\mathrm{s}} 3, \delta_{\mathrm{J} 2000}=-25^{\circ} 17^{\prime} 23^{\prime \prime}$. This position is $\sim 6^{\prime \prime}$ southeast of the dynamical center of NGC 253. A pointing accuracy of $\sim 3^{\prime \prime}$ was achieved by measuring cross scans on nearby continuum sources every $\sim 2 \mathrm{hr}$. The observations were carried out in a wobbler-switching mode with a symmetrical beam throw of $4^{\prime}$ in azimuth and a switching frequency of $0.5 \mathrm{~Hz}$.

Each of the two available SIS receivers working at $2 \mathrm{~mm}$ with orthogonal polarizations provides a $1 \mathrm{GHz}$ bandwidth. The

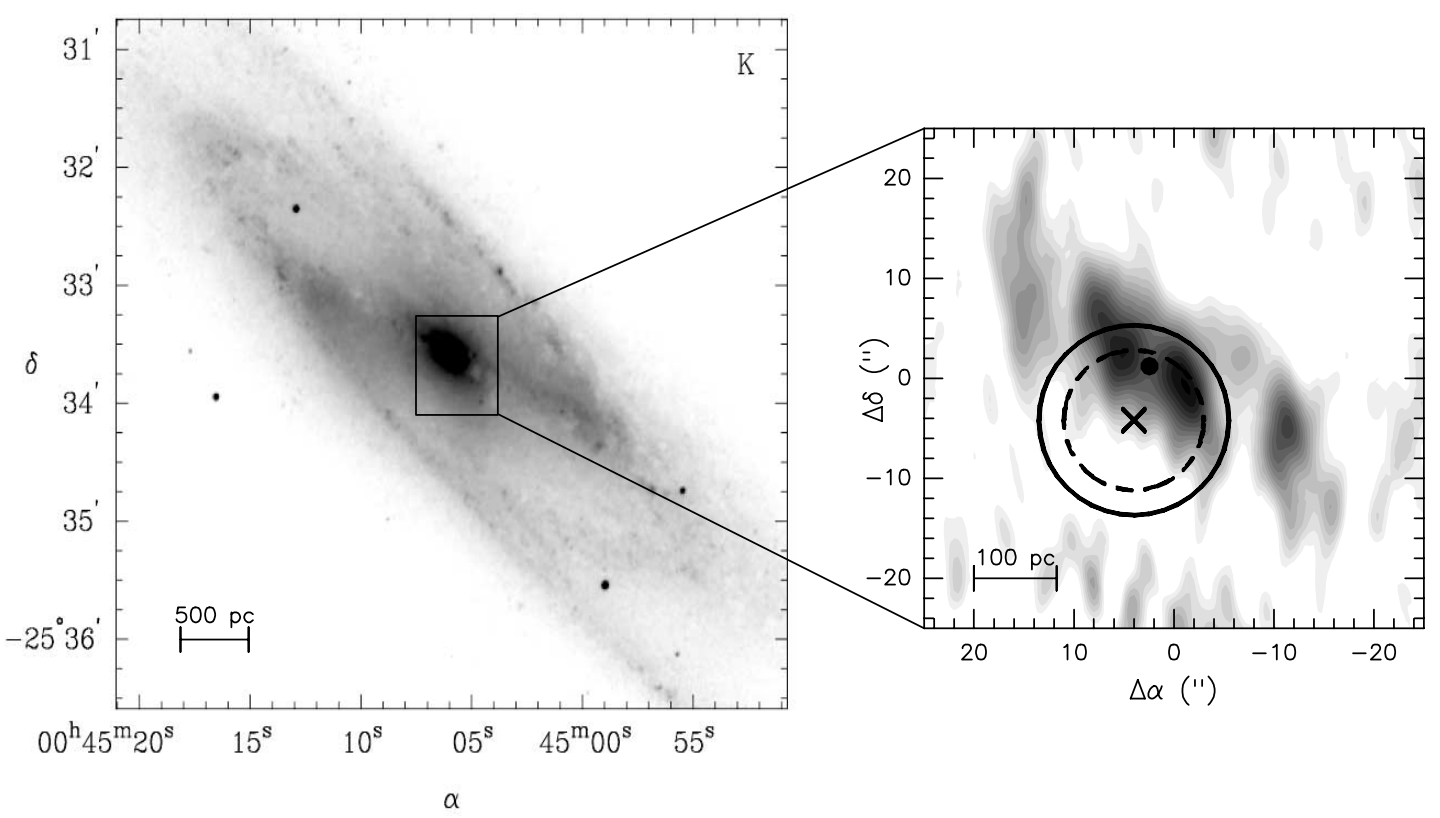

FIG. 2.-Gray-scale, K-band image of NGC 253 (left panel; Engelbracht et al. 1998) and high-resolution SiO emission (right panel; García-Burillo et al. 2000). Beam sizes of the IRAM $30 \mathrm{~m}$ telescope for the extreme frequencies observed in the survey are shown as circles on top of the $\mathrm{SiO}$ map. The continuous line shows the $19^{\prime \prime}$ beam at $129 \mathrm{GHz}$ and the dashed line the $14^{\prime \prime}$ beam at $175 \mathrm{GHz}$. A cross indicates the nominal position of the $2 \mathrm{~mm}$ scan in this work and a dot indicates the dynamical center as derived by GarcíaBurillo et al. (2000). 


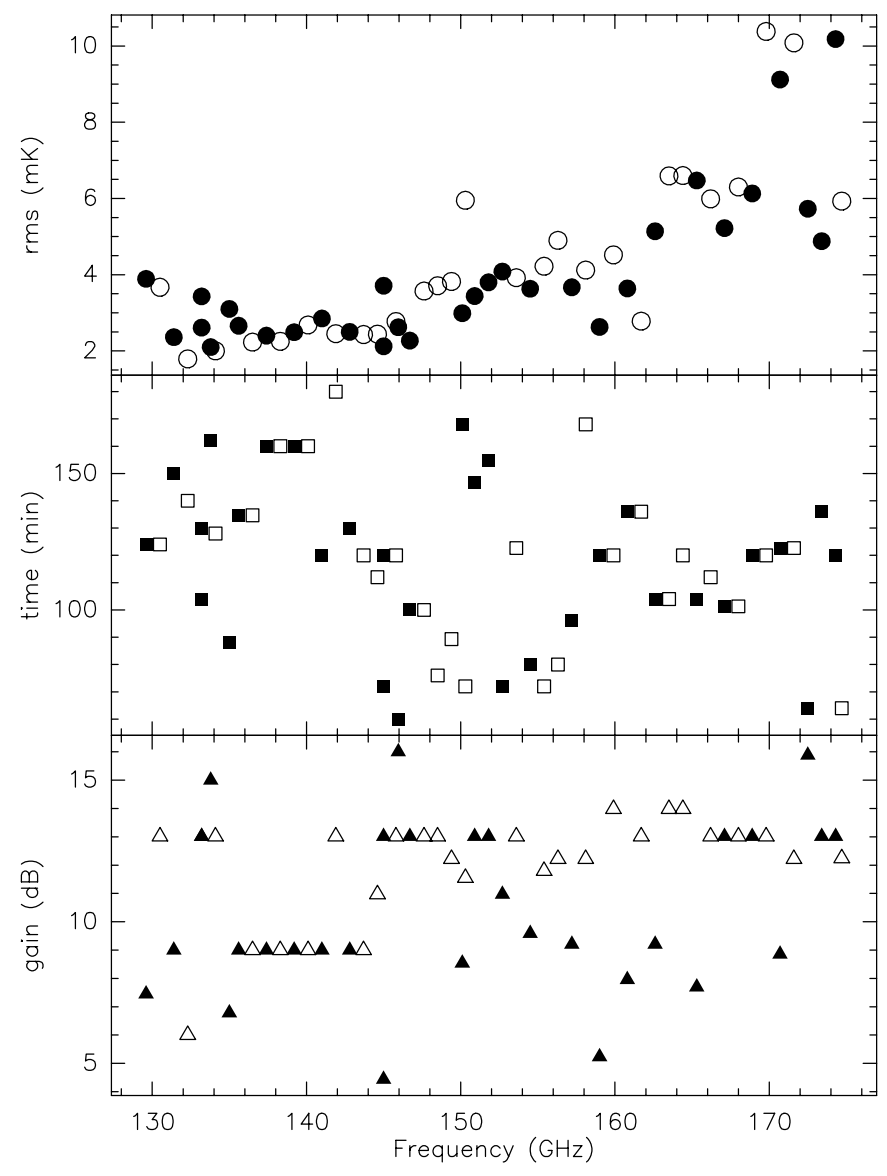

FIG. 3.- Summary of the observational parameters of individual frequency setups. Open and filled symbols refer to each of the $2 \mathrm{~mm}$ SIS receivers. Upper panel: rms of the spectrum in $7-9 \mathrm{~km} \mathrm{~s}^{-1}$ wide channels in $T_{\mathrm{mb}}$ after subtracting a linear baseline; Middle panel: Total integration time, which includes the time spent on the reference position; Lower panel: Measured rejection of the image (upper) sideband.

receivers were tuned to adjacent frequencies, with an overlap of $100 \mathrm{MHz}$, to cover an instantaneous bandwidth of $1.9 \mathrm{GHz}$ for each frequency setup. As spectrometers we used two $256 \times$ $4 \mathrm{MHz}$ filter banks, providing a velocity resolution between 7 and $9 \mathrm{~km} \mathrm{~s}^{-1}$ at the observed frequencies.

The rejection of the image band (the upper sideband) was typically $\sim 10 \mathrm{~dB}$ so that only the very few strongest lines were also detected from the image band. The image sideband rejection was calculated for each frequency setup (Fig. 3, lower panel) by measuring the difference in power between a hot and a cold load, first with full rejection of the upper sideband and then with full rejection of the lower sideband by using a Martin-Puplett interferometer. The image gain is then computed as the ratio between these two measurements. We assume this gain ratio to be constant throughout the whole $1 \mathrm{GHz}$ frequency band covered by each back end.

The spectra were calibrated with a standard dual load system. The temperature scale of the spectra is in $T_{\mathrm{MB}}$ obtained as

$$
T_{\mathrm{MB}}=\left(F_{\mathrm{eff}} / B_{\mathrm{eff}}\right) T_{\mathrm{A}}^{*},
$$

where the forward efficiency $\left(F_{\text {eff }}\right)$ is 0.93 at $2 \mathrm{~mm}$. The beam efficiency was calculated for each frequency with the Ruze function,

$$
B_{\text {eff }}=1.2 \epsilon \exp \left[-(4 \pi R \sigma / \lambda)^{2}\right],
$$

using $\epsilon=0.69, R \sigma=0.07$, and $\lambda$ in $\mathrm{mm}$.
At the latitude of Pico Veleta Observatory, NGC 253 is at elevations $>20^{\circ}$ only for about $4 \mathrm{hr}$. A complete observing session with about 120 minutes effective observing time was spent on each frequency setup, out of which half the time was spent on source. The integration time for each frequency setup is shown in the central panel of Figure 3.

The observing mode combined with the stability of the system provided high-quality baselines. Only linear baselines were subtracted from the spectra. The rms of the residuals after subtracting the fitted line profiles on each frequency setup is shown in the upper panel of Figure 3. The values are mostly on the order of $2-4 \mathrm{mK}$ in $\sim 8 \mathrm{~km} \mathrm{~s}^{-1}$ wide channels, increasing up to $\sim 6-$ $10 \mathrm{mK}$ at the highest measured frequencies.

In the scanned $46 \mathrm{GHz}$ wide band we have detected a total of 111 lines, the weakest of which have an intensity of $\sim 3 \mathrm{mK}$. The spectral density of detected lines is 2.4 features $\mathrm{GHz}^{-1}$. Since the Doppler line width in NGC 253 is about $200 \mathrm{~km} \mathrm{~s}^{-1}$, or $100 \mathrm{MHz}$, we are not limited by line blending, but by a lack of sensitivity. For targeted searches with longer integration time it may still be possible to detect and identify lines at levels of $\leq 1 \mathrm{mK}$.

Figure 4 shows the full $2 \mathrm{~mm}$ spectral line scan of NGC 253 with the line identifications superposed on the spectra. In Table 1 we are listing the parameters derived from Gaussian fits to the detected lines. We identify 25 molecular species and 3 hydrogen recombination lines.

\section{DATA ANALYSIS}

\subsection{Line Identification and Fitting}

In order to identify and fit the observed features we have used the rest frequencies from the molecular line catalogs of Lovas (1992, 2004) and Pickett et al. (1998). The existing molecular line surveys toward the Galactic center sources Sgr B2 $(\mathrm{OH})$ (Cummins et al. 1986; Turner 1989, 1991) and Sgr B2(N, M, and NW) (Nummelin et al. 1998, 2000) were also used as additional information to estimate the expected intensity of the fainter identified lines not included in the Lovas (2004) catalog.

One of the main difficulties in identifying lines from singledish observations of extragalactic sources stems from their large width, typically $\geq 100 \mathrm{~km} \mathrm{~s}^{-1}$. In addition, the emission lines from the nuclear region of NGC 253 show two velocity components, roughly at 180 and $280 \mathrm{~km} \mathrm{~s}^{-1}$, with line widths of $\sim 100$ and $110 \mathrm{~km} \mathrm{~s}^{-1}$, respectively (from CS; Martín et al. 2005). These components arise from the two main molecular lobes separated by $10^{\prime \prime}$ and located on both sides of the nucleus. As a consequence, some of the observed transitions will appear partially or totally blended. Table 1 shows the results of the Gaussian fits to all the molecular transitions identified toward the NGC 253 nuclear region. In the third column of Table 1 we have included a note to indicate whether the transition is affected by blending. Appendix A describes the fitting procedures used for the different types of blending found in our spectral line survey.

\subsection{Column Density Determination}

From the observed main beam brightness temperature of the measured molecular lines one can estimate column densities and rotation temperatures $\left(T_{\text {rot }}\right)$ for each species (see $\S \mathrm{B} 1$ for a detailed discussion). All the necessary spectroscopic information required to derive these parameters (i.e., $A_{u l}, \nu, g_{u}, E_{u}$, and $Z$ in eq. [B6]) were extracted or derived from the JPL catalog (Pickett et al. 1998).

In order to derive $T_{\text {rot }}$ and to extrapolate the column densities in the observed states to a total column density for a given molecule, more than one transition has to be measured. In the case 

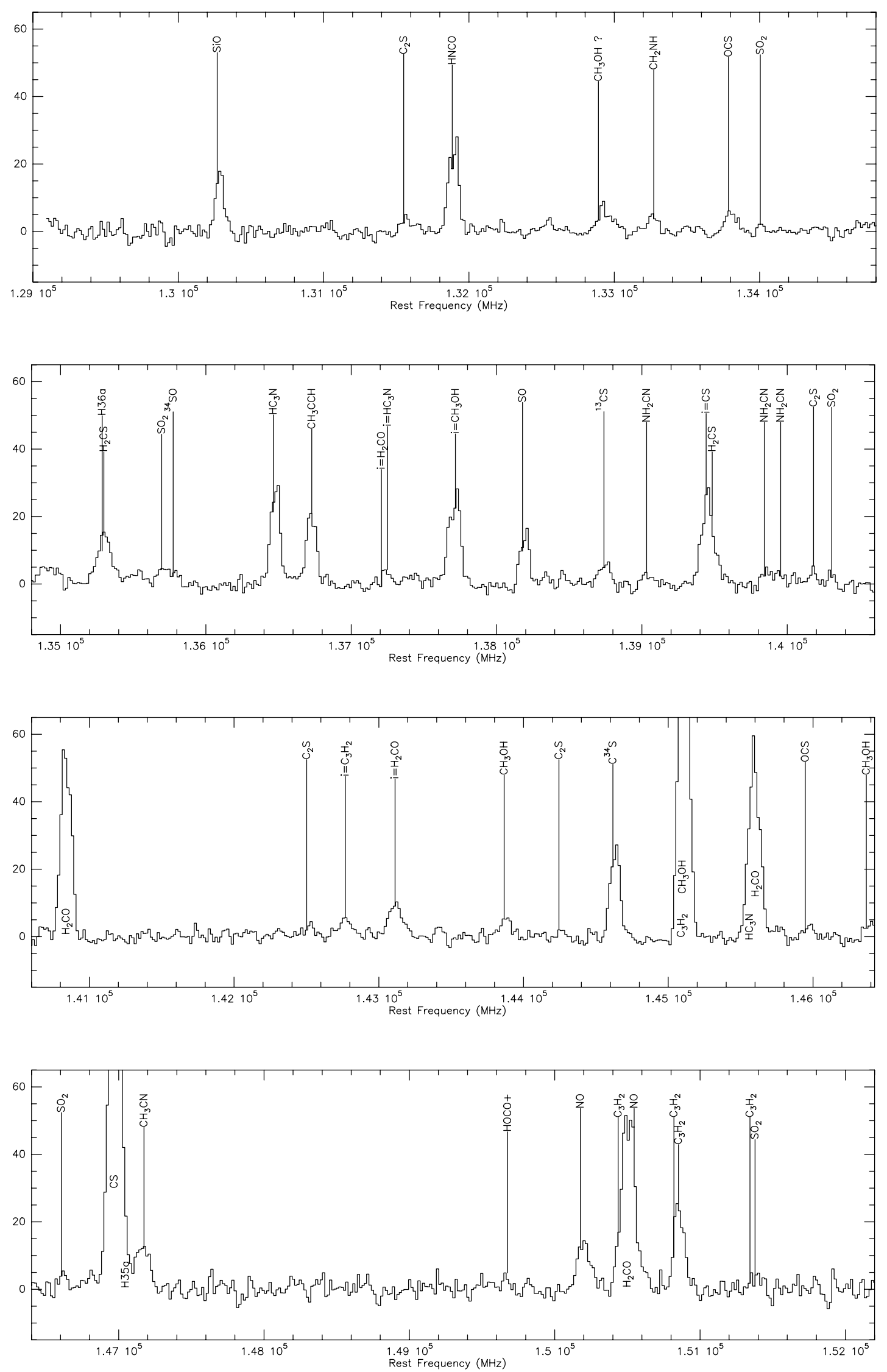

FIG. 4. $-2 \mathrm{~mm}$ spectral line survey composite toward the nuclear region of NGC 253 . The resolution has been smoothed to four channels, which is equivalent to velocity resolutions of $35-27 \mathrm{~km} \mathrm{~s}^{-1}$. Line identifications are plotted on top of the spectra. Species labeled with $i=$ molecule correspond to lines observed from the image band. 

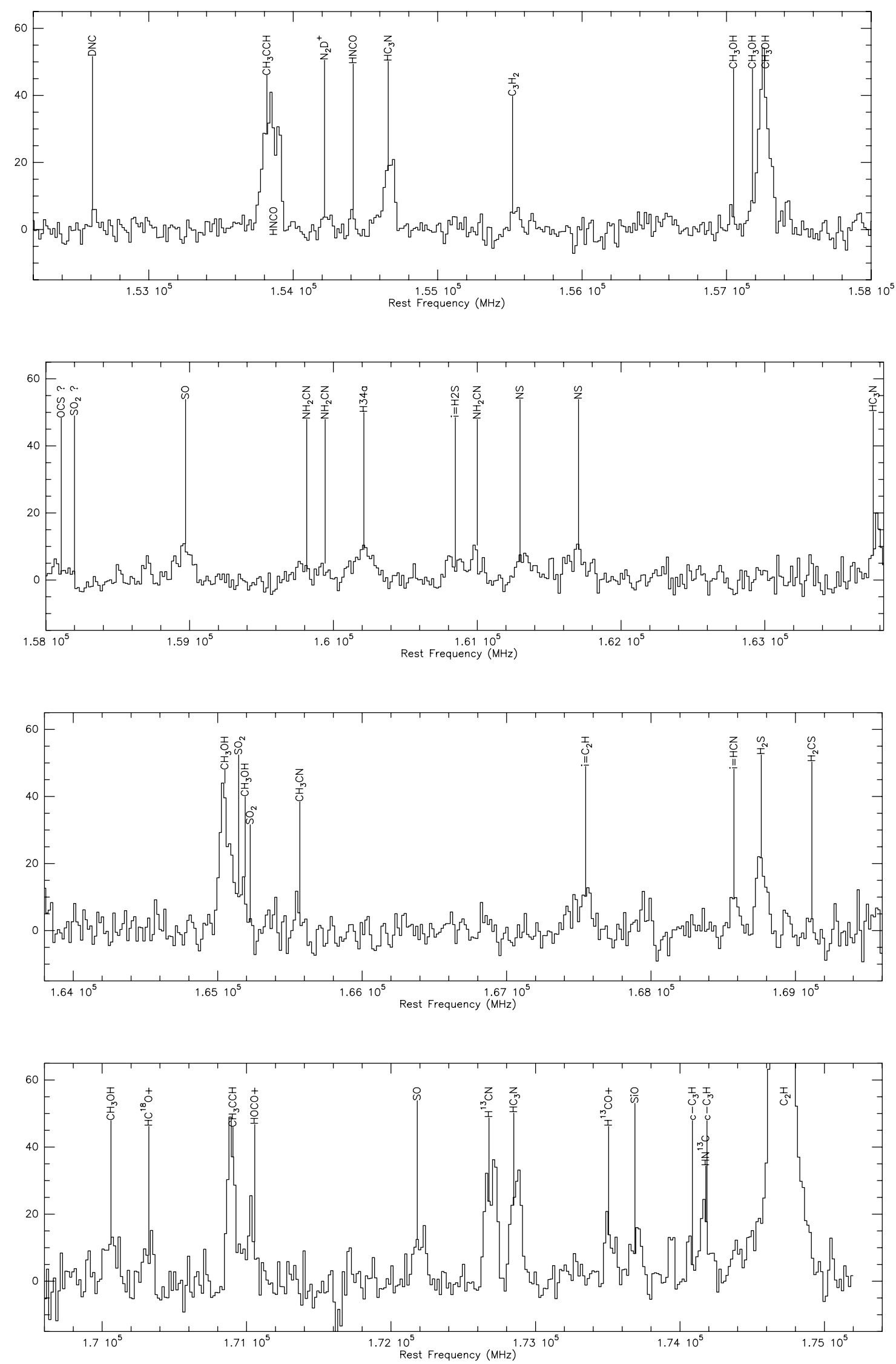

FIG. 4.-Continued 
TABLE 1

Results from Gaussian Fits to the Observed Lines

\begin{tabular}{|c|c|c|c|c|c|c|}
\hline $\begin{array}{l}\text { Molecule } \\
\text { Transition }\end{array}$ & $\begin{array}{c}\nu \\
(\mathrm{MHz})\end{array}$ & Note & $\begin{array}{c}\int T_{\mathrm{MB}} d v \\
\left(\mathrm{mK} \mathrm{km} \mathrm{s}^{-1}\right)\end{array}$ & $\begin{array}{c}V_{\mathrm{LSR}} \\
\left(\mathrm{km} \mathrm{s}^{-1}\right)\end{array}$ & $\begin{array}{c}\Delta v_{1 / 2} \\
\left(\mathrm{~km} \mathrm{~s}^{-1}\right)\end{array}$ & $\begin{array}{l}T_{\mathrm{MB}} \\
(\mathrm{mK})\end{array}$ \\
\hline \multirow[t]{2}{*}{ 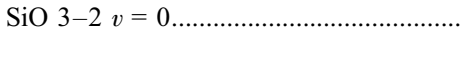 } & \multirow[t]{2}{*}{130268.6} & & $1580(50)$ & 183 & 76 & 18 \\
\hline & & & $1170(50)$ & 260 & 91 & 12 \\
\hline $\mathrm{C}_{2} \mathrm{~S} 11_{10}-10_{9} \ldots \ldots$ & 131551.9 & & $640(90)$ & 230 & 151 & 4.0 \\
\hline \multirow[t]{2}{*}{ 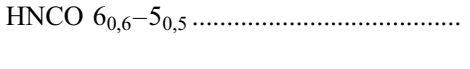 } & \multirow[t]{2}{*}{131885.7} & & $2400(150)$ & 182 & 72 & 32 \\
\hline & & & $2000(180)$ & 284 & 80 & 24 \\
\hline \multirow[t]{2}{*}{ 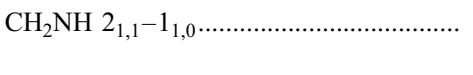 } & \multirow[t]{2}{*}{133272.1} & \multirow[t]{2}{*}{ hf } & $420(40)$ & 187 & $83^{\mathrm{b}}$ & 4.8 \\
\hline & & & $450(40)$ & 283 & $83^{\mathrm{b}}$ & 5.1 \\
\hline OCS $11-10 \ldots \ldots$ & 133785.9 & & $1220(130)$ & 212 & 200 & 5.8 \\
\hline $\mathrm{SO}_{2} 8_{2,6}-8_{1,7} \ldots$ & 134004.8 & & $300(70)$ & 242 & 91 & 3.1 \\
\hline 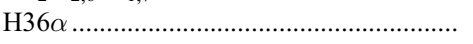 & 135286.0 & $\mathrm{~b}, \mathrm{~s}$ & $2800(\ldots)$ & $250^{\mathrm{a}}$ & $282^{\mathrm{a}}$ & 9.3 \\
\hline $\mathrm{H}_{2} \mathrm{CS} 4_{1,4}-3_{1,3} \ldots \ldots \ldots$ & 135297.8 & $\mathrm{~b}$ & $1040(90)$ & 221 & 158 & 6.2 \\
\hline 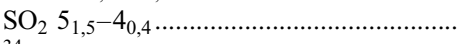 & 135696.0 & & $740(160)$ & $245^{\mathrm{a}}$ & $140^{\mathrm{a}}$ & 4.2 \\
\hline \multirow[t]{2}{*}{ 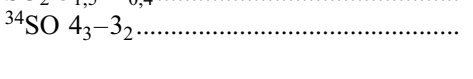 } & \multirow[t]{2}{*}{135775.3} & $\mathrm{~b}$ & $300(60)$ & $180^{\mathrm{a}}$ & $73^{\mathrm{a}}$ & 3.8 \\
\hline & & $\mathrm{b}$ & $200(70)$ & $274^{\mathrm{a}}$ & $88^{\mathrm{a}}$ & 2.1 \\
\hline \multirow{2}{*}{ 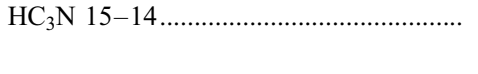 } & \multirow[t]{2}{*}{136464.4} & & $2410(130)$ & 183 & 77 & 29 \\
\hline & & & $2000(300)$ & 271 & 85 & 22 \\
\hline 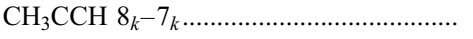 & 136728.0 & & $3410(110)$ & 255 & 158 & 20 \\
\hline \multirow{2}{*}{ 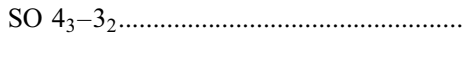 } & 138178.5 & & $1400(200)$ & 180 & 73 & 18 \\
\hline & & & $1100(200)$ & 274 & 88 & 12 \\
\hline 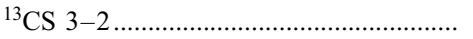 & 138739.3 & & $550(70)$ & 188 & $70^{\mathrm{b}}$ & 7.4 \\
\hline & & & $390(50)$ & 274 & $70^{\mathrm{b}}$ & 5.2 \\
\hline 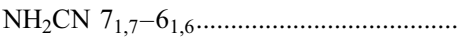 & 139032.0 & & $580(110)$ & $280^{\mathrm{a}}$ & $155^{\mathrm{a}}$ & 3.5 \\
\hline 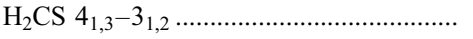 & 139483.4 & $\mathrm{~b}$ & $1340(150)$ & 221 & 176 & 7.2 \\
\hline 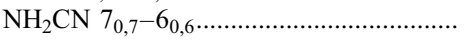 & 139842.1 & & $490(160)$ & $280^{\mathrm{a}}$ & $155^{\mathrm{a}}$ & 3.0 \\
\hline 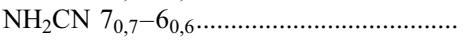 & 139954.4 & $\mathrm{~m}$ & $520(160)$ & 279 & 140 & 3.5 \\
\hline 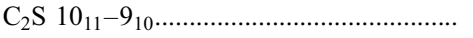 & 140180.7 & & $470(130)$ & 250 & 85 & 5.2 \\
\hline $\mathrm{SO}_{2} 6_{2,4}-6_{1,5} \ldots \ldots \ldots \ldots \ldots$ & 140306.1 & & $510(60)$ & 248 & 115 & 4.1 \\
\hline 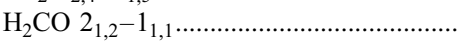 & 140839.5 & & $4250(140)$ & 186 & $104^{\mathrm{b}}$ & 38 \\
\hline & & & $6050(150)$ & 288 & $104^{\mathrm{b}}$ & 55 \\
\hline 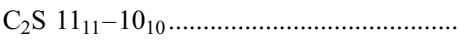 & 142501.7 & & $430(70)$ & 192 & $85^{\mathrm{a}}$ & 4.7 \\
\hline 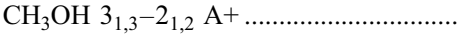 & 143865.7 & & $1000(130)$ & 225 & 168 & 5.6 \\
\hline 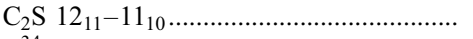 & 144244.8 & & $290(120)$ & 210 & 112 & 2.4 \\
\hline 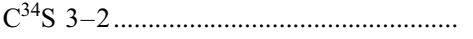 & 144617.1 & & $3000(300)$ & 188 & 105 & 27 \\
\hline & & & $1500(300)$ & 284 & 86 & 16 \\
\hline$c-\mathrm{C}_{3} \mathrm{H}_{2} 3_{1,2}-2_{2,1} \ldots \ldots \ldots \ldots$ & 145089.5 & $\mathrm{~b}, \mathrm{~s}$ & $1750(\ldots)$ & $234^{\mathrm{a}}$ & $156^{\mathrm{a}}$ & 11 \\
\hline 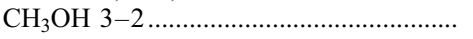 & 145103.2 & $\mathrm{~b}, \mathrm{~m}$ & $9400(200)$ & 185 & 115 & 77 \\
\hline & & & $5760(190)$ & 294 & 96 & 57 \\
\hline 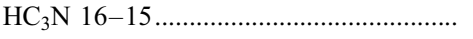 & 145560.9 & $\mathrm{~b}, \mathrm{~s}$ & $2100(\ldots)$ & $183^{\mathrm{a}}$ & $77^{\mathrm{a}}$ & 26 \\
\hline & & $\mathrm{b}, \mathrm{s}$ & $1700(\ldots)$ & $271^{\mathrm{a}}$ & $85^{\mathrm{a}}$ & 19 \\
\hline 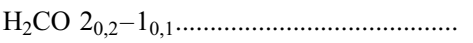 & 145602.9 & $\mathrm{~b}$ & $3400(100)$ & 177 & $90^{\mathrm{b}}$ & 35 \\
\hline & & $\mathrm{b}$ & $3300(100)$ & 283 & $90^{\mathrm{b}}$ & 34 \\
\hline 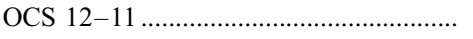 & 145946.8 & & $580(170)$ & $212^{\mathrm{a}}$ & $210^{\mathrm{a}}$ & 2.6 \\
\hline 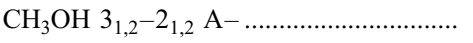 & 146368.3 & & $1020(150)$ & 196 & 139 & 6.9 \\
\hline 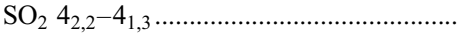 & 146605.5 & & $800(170)$ & 241 & 148 & 5.1 \\
\hline 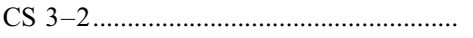 & 146969.0 & $\mathrm{~b}$ & $11900(200)$ & 185 & 100 & 111 \\
\hline & & $\mathrm{b}$ & $13700(200)$ & 288 & 117 & 110 \\
\hline $\mathrm{H} 35 \alpha \ldots \ldots \ldots \ldots \ldots \ldots \ldots \ldots$ & 147046.8 & $\mathrm{~b}, \mathrm{~s}$ & $2800(\ldots)$ & $250^{\mathrm{a}}$ & $282^{\mathrm{a}}$ & 9.3 \\
\hline 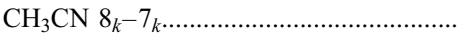 & 147174.5 & $\mathrm{~b}, \mathrm{~m}$ & $2040(180)$ & 258 & 165 & 12 \\
\hline 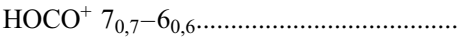 & 149675.8 & & $410(110)$ & 281 & 68 & 5.6 \\
\hline 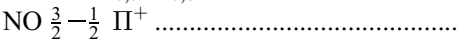 & 150176.5 & hf & $3400(500)$ & 244 & 158 & 10 \\
\hline 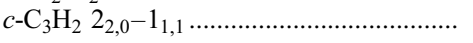 & 150436.5 & $\mathrm{~b}, \mathrm{~s}$ & $600(\ldots)$ & $234^{\mathrm{a}}$ & $156^{\mathrm{a}}$ & 3.6 \\
\hline 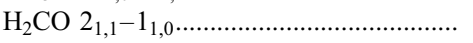 & 150498.3 & $\mathrm{~b}$ & $5300(100)$ & 180 & $99^{\mathrm{b}}$ & 50 \\
\hline & & $\mathrm{b}$ & $5100(100)$ & 286 & $99^{\mathrm{b}}$ & 48 \\
\hline 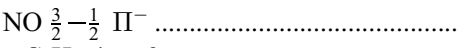 & 150546.5 & hf, b, s & $3400(\ldots)$ & $244^{\mathrm{a}}$ & $158^{\mathrm{a}}$ & 10 \\
\hline 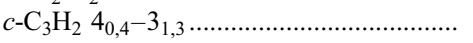 & 150820.6 & $\mathrm{~b}$ & $1150(\ldots)$ & $234^{\mathrm{b}}$ & $156^{\mathrm{b}}$ & 7.0 \\
\hline 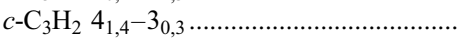 & 150851.9 & $\mathrm{~b}$ & $3380(110)$ & $234^{\mathrm{b}}$ & $156^{\mathrm{b}}$ & 20 \\
\hline 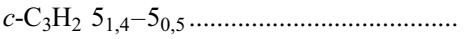 & 151343.8 & $\mathrm{~b}, \mathrm{~s}$ & $165(\ldots)$ & $234^{\mathrm{a}}$ & $156^{\mathrm{a}}$ & 1.0 \\
\hline 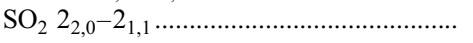 & 151378.6 & $\mathrm{~b}$ & $590(170)$ & 225 & 158 & 3.5 \\
\hline 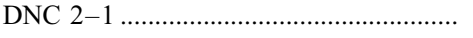 & 152609.7 & & $490(160)$ & 225 & 61 & 7.5 \\
\hline 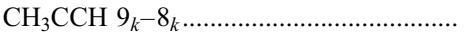 & 153817.2 & $\mathrm{~b}$ & $4300(200)$ & 271 & 157 & 26 \\
\hline 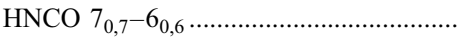 & 153865.0 & & $2660(150)$ & 183 & 72 & 34 \\
\hline & & $\mathrm{b}, \mathrm{s}$ & $2240(\ldots)$ & $284^{\mathrm{a}}$ & $80^{\mathrm{a}}$ & 26 \\
\hline 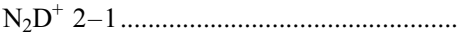 & 154217.0 & & $570(170)$ & 219 & 120 & 4.5 \\
\hline 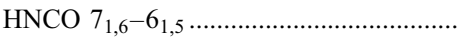 & 154414.7 & & $540(110)$ & $283^{\mathrm{a}}$ & $80^{\mathrm{a}}$ & 6.4 \\
\hline 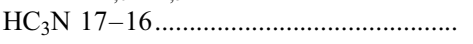 & 154657.3 & & $1640(120)$ & 191 & $72^{\mathrm{b}}$ & 21 \\
\hline & & & $1400(110)$ & 272 & $72^{b}$ & 18 \\
\hline
\end{tabular}


TABLE 1-Continued

\begin{tabular}{|c|c|c|c|c|c|c|}
\hline $\begin{array}{l}\text { Molecule } \\
\text { Transition }\end{array}$ & $\begin{array}{c}\nu \\
(\mathrm{MHz})\end{array}$ & Note & $\underset{\left(\mathrm{mK} \mathrm{km} \mathrm{s} \mathrm{km}^{-1}\right)}{\int T_{\mathrm{MB}} d v}$ & $\begin{array}{c}V_{\mathrm{LSR}} \\
\left(\mathrm{km} \mathrm{s}^{-1}\right)\end{array}$ & $\begin{array}{c}\Delta v_{1 / 2} \\
\left(\mathrm{~km} \mathrm{~s}^{-1}\right)\end{array}$ & $\begin{array}{l}T_{\mathrm{MB}} \\
(\mathrm{mK})\end{array}$ \\
\hline 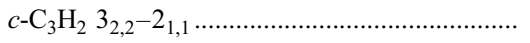 & 155518.2 & & $820(180)$ & 205 & 118 & 6.6 \\
\hline 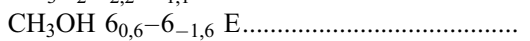 & 157048.6 & & $540(120)$ & 275 & $103^{\mathrm{b}}$ & 5.0 \\
\hline $\mathrm{CH}_{3} \mathrm{OH} 5_{0,5} 5_{-1,5} \mathrm{E}$ & 157178.9 & $\mathrm{~b}$ & $960(150)$ & 242 & $103^{\mathrm{b}}$ & 8.8 \\
\hline 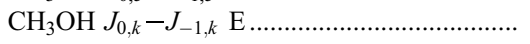 & 157270.7 & $\mathrm{~b}, \mathrm{~m}$ & $2110(160)$ & 183 & $103^{\mathrm{b}}$ & 19 \\
\hline$J=1 \ldots 4 ; k=J$ & & & $5700(200)$ & 290 & $103^{\mathrm{b}}$ & 52 \\
\hline \multirow[t]{2}{*}{ 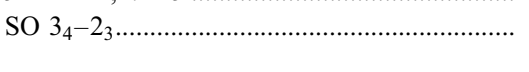 } & 158971.7 & & $700(200)$ & $180^{\mathrm{a}}$ & 92 & 7.2 \\
\hline & & $\mathrm{b}$ & $1000(200)$ & $274^{\mathrm{a}}$ & 84 & 11 \\
\hline $\mathrm{NH}_{2} \mathrm{CN} 8_{0,8}-7_{0,7} \ldots$ & 159814.6 & & $750(180)$ & $283^{\mathrm{b}}$ & $155^{\mathrm{b}}$ & 4.5 \\
\hline 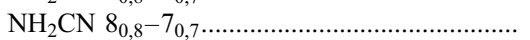 & 159942.7 & $\mathrm{~m}$ & $700(200)$ & $283^{\mathrm{b}}$ & $158^{\mathrm{b}}$ & 4.1 \\
\hline $\mathrm{H} 34 \alpha$ & 160211.5 & & $2800(200)$ & 250 & 282 & 9.3 \\
\hline 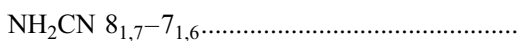 & 161000.3 & $\mathrm{~b}$ & $1430(170)$ & 280 & $155^{\mathrm{a}}$ & 8.6 \\
\hline NS $\frac{7}{2}-\frac{5}{2} e$ & 161297.2 & hf & $1800(500)$ & 199 & 224 & 3.1 \\
\hline 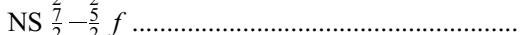 & 161697.2 & $\mathrm{hf}$ & $2400(400)$ & 249 & 278 & 3.4 \\
\hline \multirow[t]{2}{*}{$\mathrm{HC}_{3} \mathrm{~N} 18-17 \ldots$} & 163753.4 & & $1500(400)$ & $185^{\mathrm{a}}$ & 73 & 20 \\
\hline & & & $700(300)$ & $272^{\mathrm{a}}$ & 98 & 6.6 \\
\hline 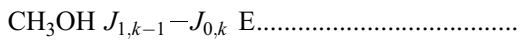 & 165050.1 & $\mathrm{~b}, \mathrm{~m}$ & $1700(400)$ & 169 & 72 & 22 \\
\hline$J=1 \ldots 3 ; k=J$ & & & $4700(400)$ & 273 & 100 & 45 \\
\hline 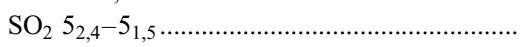 & 165144.6 & $\mathrm{~b}, \mathrm{~s}$ & $640(\ldots)$ & $248^{\mathrm{a}}$ & $115^{\mathrm{a}}$ & 5.2 \\
\hline 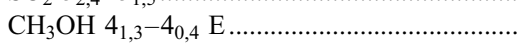 & 165190.5 & $\mathrm{~b}$ & $900(200)$ & 291 & $90^{\mathrm{a}}$ & 9.1 \\
\hline $\mathrm{SO}_{2} 7_{1,7}-6_{0,6} \ldots \ldots$ & 165225.4 & $\mathrm{~b}, \mathrm{~s}$ & $900(\ldots)$ & $248^{\mathrm{a}}$ & $115^{\mathrm{a}}$ & 7.4 \\
\hline $\mathrm{CH}_{3} \mathrm{CN} 9_{\mathrm{k}}-8_{\mathrm{k}}$ & 165568.9 & $\mathrm{~b}, \mathrm{~m}$ & $1200(300)$ & $258^{\mathrm{a}}$ & $166^{\mathrm{a}}$ & 7.0 \\
\hline \multirow[t]{2}{*}{ 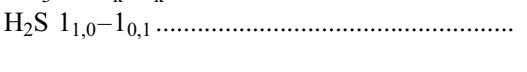 } & 168762.7 & & $1520(180)$ & 180 & 100 & 14 \\
\hline & & & $2250(80)$ & 275 & 88 & 24 \\
\hline $\mathrm{H}_{2} \mathrm{CS} 5_{1,5}-4_{1,4} \ldots \ldots \ldots \ldots$ & 169113.5 & & $970(200)$ & 270 & $165^{\mathrm{a}}$ & 5.5 \\
\hline 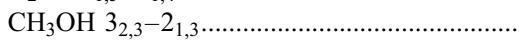 & 170060.6 & & $2400(400)$ & 248 & 178 & 12 \\
\hline \multirow[t]{2}{*}{$\mathrm{HC}^{18} \mathrm{O}^{+} 2-1$} & 170322.7 & & $500(300)$ & 206 & 46 & 11 \\
\hline & & & $500(200)$ & 285 & 49 & 10 \\
\hline $\mathrm{CH}_{3} \mathrm{CCH} 10_{k}-9_{k} \ldots$ & 170905.6 & & $5100(500)$ & 275 & 102 & 47 \\
\hline 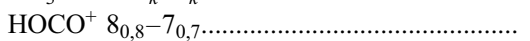 & 171055.9 & $\mathrm{~b}$ & $1700(300)$ & 295 & 66 & 25 \\
\hline \multirow[t]{2}{*}{$\mathrm{SO} 4_{4}-3_{3} \ldots \ldots \ldots$} & 172181.4 & & $1300(300)$ & $180^{\mathrm{a}}$ & 87 & 14 \\
\hline & & & $900(300)$ & $274^{\mathrm{a}}$ & 74 & 11 \\
\hline \multirow[t]{2}{*}{$\mathrm{H}^{13} \mathrm{CN} 2_{\mathrm{k}}-1_{\mathrm{k}}$} & 172677.9 & hf & $3200(200)$ & 181 & 74 & 40 \\
\hline & & & $2400(200)$ & 279 & 66 & 34 \\
\hline \multirow{2}{*}{ 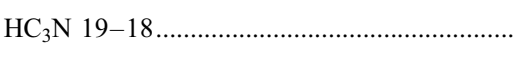 } & 172849.3 & & $2800(400)$ & 184 & 74 & 35 \\
\hline & & & $1800(400)$ & 269 & 75 & 22 \\
\hline \multirow[t]{2}{*}{$\mathrm{H}^{13} \mathrm{CO}^{+} 2-1$} & 173506.7 & & $860(90)$ & 170 & $60^{\mathrm{b}}$ & 14 \\
\hline & & & $1450(100)$ & 269 & $60^{\mathrm{b}}$ & 23 \\
\hline \multirow{2}{*}{$\mathrm{SiO} 4-3 v=0$} & 173688.3 & & $1070(110)$ & $184^{\mathrm{a}}$ & $76^{\mathrm{a}}$ & 13 \\
\hline & & & $1000(120)$ & $270^{\mathrm{a}}$ & $91^{\mathrm{a}}$ & 10 \\
\hline \multirow{2}{*}{ 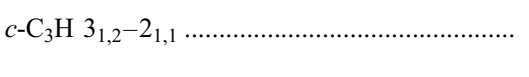 } & 174086.1 & $\mathrm{hf}$ & $900(200)$ & $180^{\mathrm{a}}$ & $41^{\mathrm{b}}$ & 6.5 \\
\hline & & & $1600(500)$ & $280^{\mathrm{a}}$ & 40 & 12 \\
\hline $\mathrm{HN}^{13} \mathrm{C} 2-1 \ldots$. & 174179.4 & & $1050(170)$ & 271 & 60 & 16 \\
\hline \multirow[t]{2}{*}{$\mathrm{C}_{2} \mathrm{H} 2-1$} & 174663.2 & $\mathrm{hf}$ & $30000(6000)$ & 162 & 108 & 82 \\
\hline & & & $27000(6000)$ & 282 & 104 & 88 \\
\hline
\end{tabular}

Notes.-See Appendix A for details: (b) blended line; (s) synthetic Gaussian to isolate a given feature (see text for details on the parameters used for deriving these profiles for each species); (hf) hyperfine structure (frequency and intensity refers to the main component of the group); (m) multitransition line (frequency refers to the main component of the group).

${ }^{a}$ Parameter fixed in the Gaussian fit.

b Parameters forced to have the same value in the Gaussian fit.

that only one transition was detected in the $2 \mathrm{~mm}$ scan, an educated guess of the rotation temperature was made (see Appendix C).

Table 2 shows the derived total column densities and rotation temperatures for the detected species in NGC 253. Source-averaged abundances are also given, where a source-averaged molecular hydrogen column density $N\left(\mathrm{H}_{2}\right)=6.7 \times 10^{22} \mathrm{~cm}^{-2}$ has been assumed (see $\S$ 4.4.1).

\subsection{Uncertainty in Column Densities and Rotation Temperatures}

The main source of uncertainty, when deriving the column densities and rotation temperatures, arises from the uncertainty of the extent of the emitting regions. The source-averaged bright- ness temperature $\left(T_{\mathrm{B}}\right)$ can be estimated from the measured main beam brightness temperature as

$$
T_{\mathrm{B}}=\eta_{\mathrm{bf}}^{-1} T_{\mathrm{MB}},
$$

where, in the approximation of a Gaussian source distribution of size $\theta_{s}$ observed with a Gaussian beam of size $\theta_{b}$, the beam filling factor $\eta_{\mathrm{bf}}=\theta_{s}^{2} /\left(\theta_{s}^{2}+\theta_{b}^{2}\right)$ accounts for the dilution effect due to the coupling between the source and the telescope beam.

In order to correct for beam dilution in the nuclear region of NGC 253, Mauersberger et al. (2003) smoothed the high-resolution interferometric CS map from Peng et al. (1996) to convert the three observed transitions of CS by Mauersberger \& Henkel (1989) 
TABLE 2

Physical Parameters Derived for All Detected Species

\begin{tabular}{|c|c|c|c|c|}
\hline Molecule & $\begin{array}{c}\text { Velocity } \\
\left(\mathrm{km} \mathrm{s}^{-1}\right)\end{array}$ & $\begin{array}{c}N^{\mathrm{a}} \\
\left(\mathrm{cm}^{-2}\right)\end{array}$ & $\begin{array}{l}T_{\text {rot }} \\
(\mathrm{K})\end{array}$ & $\begin{array}{r}{[X] /\left[\mathrm{H}_{2}\right]} \\
\left(\times 10^{-9}\right)\end{array}$ \\
\hline \multirow[t]{2}{*}{$\mathrm{SiO}$} & 180 & $5.0(1.0) \times 10^{12}$ & $7.4(0.7)$ & 0.07 \\
\hline & 270 & $3.6(0.9) \times 10^{12}$ & $8.7(1.2)$ & 0.05 \\
\hline $\mathrm{C}_{2} \mathrm{~S} \ldots \ldots$ & & $1.4(1.2) \times 10^{13}$ & $24(9)$ & 0.2 \\
\hline $\mathrm{NH}_{2} \mathrm{CN}$ & & $1.2(0.5) \times 10^{13}$ & $67(13)$ & 0.2 \\
\hline $\mathrm{CH}_{3} \mathrm{CN}$ & & $2.0(0.6) \times 10^{13}$ & $9.6(0.7)$ & 0.3 \\
\hline \multirow[t]{2}{*}{$c-\mathrm{C}_{3} \mathrm{H}$} & 130 & $8.1(1.6) \times 10^{12}$ & 12 & 0.1 \\
\hline & 280 & $1.5(0.3) \times 10^{13}$ & 12 & 0.2 \\
\hline$c-\mathrm{C}_{3} \mathrm{H}_{2} \ldots \ldots \ldots \ldots \ldots$ & & $3.0(6.0) \times 10^{13}$ & $9(8)$ & 0.4 \\
\hline $\mathrm{NS}^{\mathrm{c}}$ & & $4.3(0.6) \times 10^{13}$ & $7.2(1.0)$ & 0.6 \\
\hline \multirow{2}{*}{$\mathrm{HC}_{3} \mathrm{~N}$} & 180 & $1.8(0.5) \times 10^{13}$ & $33(4)$ & 0.3 \\
\hline & 270 & $1.9(0.6) \times 10^{13}$ & $24(3)$ & 0.3 \\
\hline $\mathrm{HOCO}^{+} \ldots \ldots \ldots \ldots \ldots$ & & $2.6(1.8) \times 10^{13}$ & 12 & 0.4 \\
\hline $\mathrm{H}_{2} \mathrm{CS}$ & & $4.4(4.5) \times 10^{13}$ & $11(4)$ & 0.6 \\
\hline $\mathrm{SO}_{2} \ldots \ldots \ldots \ldots \ldots \ldots \ldots$ & & $5.0(2.0) \times 10^{13}$ & $15(2)$ & 0.7 \\
\hline \multirow[t]{2}{*}{$\mathrm{H}_{2} \mathrm{~S}$} & 180 & $2.3(1.1) \times 10^{13}$ & 12 & 0.3 \\
\hline & 275 & $3.5(1.4) \times 10^{13}$ & 12 & 0.5 \\
\hline \multirow[t]{2}{*}{$\mathrm{CH}_{2} \mathrm{NH} \ldots \ldots \ldots \ldots \ldots$} & 190 & $2.5(1.1) \times 10^{13}$ & 12 & 0.4 \\
\hline & 300 & $2.8(0.9) \times 10^{13}$ & 12 & 0.4 \\
\hline $\mathrm{HNC}^{\mathrm{c}}$ & 270 & $7.2(1.2) \times 10^{13}$ & 12 & 1 \\
\hline \multirow[t]{2}{*}{$\mathrm{SO}$} & 180 & $4.5(3.3) \times 10^{13}$ & $40(24)$ & 0.7 \\
\hline & 270 & $3.1(2.8) \times 10^{13}$ & $34(24)$ & 0.5 \\
\hline \multirow[t]{2}{*}{ 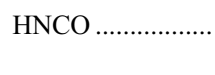 } & 180 & $5.7(2.7) \times 10^{13}$ & $23(6)$ & 0.8 \\
\hline & 280 & $4.8(2.5) \times 10^{13}$ & 23 & 0.7 \\
\hline \multirow[t]{2}{*}{$\mathrm{HCO}^{+\mathrm{c}}$} & 170 & $3.9(0.8) \times 10^{13}$ & 12 & 0.6 \\
\hline & 270 & $6.4(1.2) \times 10^{13}$ & 12 & 1 \\
\hline \multirow[t]{2}{*}{$\mathrm{H}_{2} \mathrm{CO}$} & 180 & $6.9(0.9) \times 10^{13}$ & $27(2)$ & 1 \\
\hline & 285 & $9.0(1.7) \times 10^{13}$ & $34(4)$ & 1 \\
\hline $\mathrm{OCS}^{\mathrm{d}}$ & & $2.5(0.3) \times 10^{14}$ & $17(2)$ & 4 \\
\hline \multirow[t]{2}{*}{$\mathrm{HCN}^{\mathrm{c}}$} & 180 & $1.8(0.3) \times 10^{14}$ & 12 & 2 \\
\hline & 280 & $1.4(0.2) \times 10^{14}$ & 12 & 2 \\
\hline \multirow[t]{2}{*}{$\mathrm{CS}^{\mathrm{c}, \mathrm{d}}$} & 180 & $2.0(0.2) \times 10^{14}$ & $9.7(0.4)$ & 3 \\
\hline & 280 & $1.4(0.2) \times 10^{14}$ & $10.0(0.2)$ & 2 \\
\hline $\mathrm{CH}_{3} \mathrm{CCH} \ldots \ldots \ldots \ldots$ & & $4.3(0.2) \times 10^{14}$ & $63(20)$ & 6 \\
\hline $\mathrm{CH}_{3} \mathrm{OH} \ldots \ldots \ldots \ldots$ & & $8.3(0.3) \times 10^{14}$ & $11.6(0.2)$ & 12 \\
\hline \multirow[t]{2}{*}{$\mathrm{C}_{2} \mathrm{H}$} & 160 & $6.8(1.1) \times 10^{14}$ & 12 & 10 \\
\hline & 280 & $5.6(0.9) \times 10^{14}$ & 12 & 8 \\
\hline $\mathrm{NO}^{\mathrm{d}}$ & & $4.0(0.4) \times 10^{15}$ & $6(2)$ & 60 \\
\hline
\end{tabular}

NoтE.-Source-averaged column densities, rotation temperature, and abundances relative to $\mathrm{H}_{2}$.

${ }^{\text {a }}$ Errors derived from the rotation diagrams without beam filling factor considerations (see $\S 3.3)$.

b Assumed $N\left(\mathrm{H}_{2}\right)=6.7 \times 10^{22} \mathrm{~cm}^{-2}$. See Table 6 .

${ }^{\text {c }}$ Calculated from the observed ${ }^{13} \mathrm{C}$ isotope transition with ${ }^{12} \mathrm{C} /{ }^{13} \mathrm{C} \sim 40$ from Henkel et al. (1993).

${ }^{\mathrm{d}}$ Using additional transitions measured by Martín et al. (2003, 2005).

to a common angular resolution of $32^{\prime \prime}$. Since we know the intensities of the CS lines with the original resolution $\left(T_{\mathrm{MB}}\right)$ and those corrected to a $32^{\prime \prime}$ beam $\left(T_{\mathrm{MB}}^{32^{\prime \prime}}\right)$ we can derive the beam filling factor. From the expression $\eta_{\mathrm{bf}}^{32^{\prime \prime}} T_{\mathrm{MB}}=\eta_{\mathrm{bf}} T_{\mathrm{MB}}^{32^{\prime \prime}}$ we estimate the extent of the emitting region to be $\theta_{s}=23^{\prime \prime}, 21^{\prime \prime}$, and $19^{\prime \prime}$ for the CS $J=2-1, J=3-2$, and $J=5-4$ transitions, respectively. In our analysis we will then consider an equivalent source size of $20^{\prime \prime}$ to convert our main beam brightness temperature to sourceaveraged brightness temperature.

The other main source of uncertainty stems from the assumption that all the observed species arise from the same volume. The only way to confirm this would be high-resolution observations of different transitions of several species. The interferometric map of the $J=2-1$ line of CS by Peng et al. (1996) shows that most of the emission of this molecule is concentrated within a radius of $\sim 20^{\prime \prime}$. The CS emission is seen to be clumped

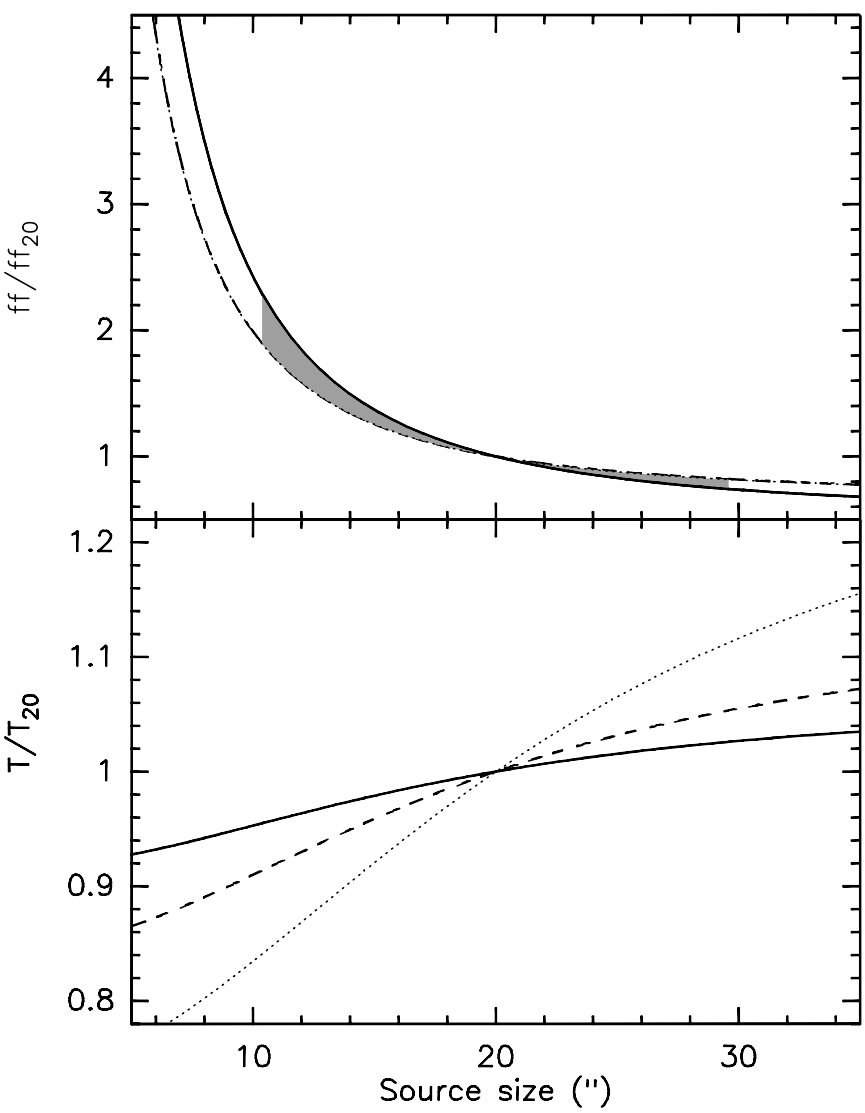

FIG. 5.-Upper panel: Variation of the filling factor normalized to that corresponding to a source extent of $20^{\prime \prime}$ as a function of source size. The solid line corresponds to the beam at $129 \mathrm{GHz}$ and the dashed line to the beam at 175 GHz. The shaded area highlights values with source sizes between 10 and $30^{\prime \prime}$. Lower panel: Effect of the assumed source size on the rotation temperature determined from rotation diagrams relative to the assumed $T_{\text {rot }}=15 \mathrm{~K}$ for $\theta_{s}=20^{\prime \prime}$ with two transitions measured at 129 and $175 \mathrm{GHz}$, where the involved levels are separated by 60 (solid line), 30 (dashed line) and $15 \mathrm{~K}$ (dotted line).

into four large main molecular cloud complexes symmetrically distributed with respect to the dynamical center and roughly aligned with the molecular bar. A recent high-resolution multiline study carried out toward IC 342 (Meier \& Turner 2005) shows clear differences in the distribution of several important molecular tracers. Toward NGC 253, however, high-resolution maps of molecular transitions requiring different excitation conditions such as $\mathrm{HCN}, \mathrm{CO}, \mathrm{SiO}, \mathrm{H}^{13} \mathrm{CO}^{+}$, and $\mathrm{NH}_{3}$ (Paglione et al. 1995, 2004; García-Burillo et al. 2000; Ott et al. 2005) show very similar distributions, in good agreement with that of CS. Therefore, it is plausible that for NGC 253, as long as the bulk of the emission arises from the area confined by the starburst, the extent of the emission of different molecules is similar.

We now address the question of how an error in the assumed source size will affect our determination of physical parameters. As derived from equation (B1) (see $\S \mathrm{B} 1$ ), the calculated total column density is affected by the filling factor through $T_{\mathrm{B}}$. The upper panel in Figure 5 shows the variation of the inverse of the filling factor (i.e., the conversion factor from $T_{\mathrm{MB}}$ to $T_{\mathrm{B}}$ from eq. [3]) as a function of source size, normalized to the value for a source of $20^{\prime \prime}$. This value represents, in a first approximation, the factor by which we should multiply our derived column densities if the source extent would be different from that assumed. We have considered the extreme cases of the beam sizes at 129 and $175 \mathrm{GHz}$. The gray shaded area corresponds to source sizes between $10^{\prime \prime}$ and $30^{\prime \prime}$, where we expect the source size to be 
TABLE 3

Known Extragalactic Molecules (See § 4.1)

\begin{tabular}{|c|c|c|c|}
\hline Molecule & $\begin{array}{c}\text { Publication } \\
\text { Year }\end{array}$ & Telescope & Reference \\
\hline 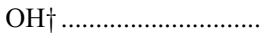 & 1971 & OVRO & 1 \\
\hline $\mathrm{H}_{2} \mathrm{CO} \ldots \ldots \ldots \ldots \ldots \ldots$ & 1974 & $64 \mathrm{~m}$ & 2 \\
\hline $\mathrm{CO} \dagger \ldots \ldots \ldots \ldots \ldots \ldots$ & 1975 & $11 \mathrm{~m}$ & 3 \\
\hline 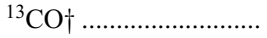 & 1975 & $11 \mathrm{~m}$ & 4 \\
\hline $\mathrm{H}_{2} \mathrm{O} \dagger \ldots \ldots \ldots \ldots \ldots \ldots \ldots \ldots$ & 1977 & $100 \mathrm{~m}$ & 5 \\
\hline 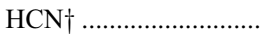 & 1977 & $11 \mathrm{~m}$ & 6 \\
\hline $\mathrm{H}_{2} \dagger \ldots \ldots \ldots \ldots \ldots \ldots \ldots \ldots \ldots \ldots \ldots$ & 1978 & $2.3 \mathrm{~m}$ & 7 \\
\hline $\mathrm{NH}_{3} \dagger \ldots \ldots \ldots \ldots \ldots \ldots \ldots \ldots \ldots \ldots \ldots \ldots \ldots \ldots \ldots \ldots$ & 1979 & $100 \mathrm{~m}$ & 8 \\
\hline $\mathrm{HCO}^{+} \dagger \ldots \ldots \ldots \ldots \ldots \ldots$ & 1979 & $7 \mathrm{~m}$ & 9 \\
\hline 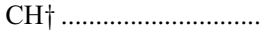 & 1980 & $64 \mathrm{~m}$ & 10 \\
\hline 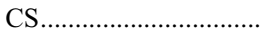 & 1985 & $7 \mathrm{~m}$ & 11 \\
\hline $\mathrm{C}_{3} \mathrm{H}_{2} \ldots \ldots \ldots \ldots \ldots \ldots \ldots$ & 1986 & $43 \mathrm{~m}$ & 12 \\
\hline $\mathrm{CH}^{+} \dagger \ldots \ldots \ldots \ldots \ldots \ldots \ldots \ldots \ldots \ldots \ldots$ & 1987 & $1.4 \mathrm{~m}$ & 13 \\
\hline $\mathrm{CH}_{3} \mathrm{OH} \ldots \ldots \ldots \ldots \ldots \ldots \ldots \ldots \ldots \ldots \ldots$ & 1987 & $30 \mathrm{~m}$ & 14 \\
\hline 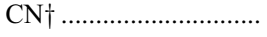 & 1988 & $30 \mathrm{~m}$ & 15 \\
\hline $\mathrm{C}_{2} \mathrm{H}$ & 1988 & $30 \mathrm{~m}$ & 15 \\
\hline 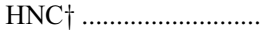 & 1988 & $30 \mathrm{~m}$ & 15 \\
\hline $\mathrm{HC}_{3} \mathrm{~N}$ & 1988 & $30 \mathrm{~m}$ & 15,16 \\
\hline 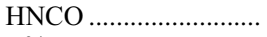 & 1989 & $30 \mathrm{~m}$ & 17,18 \\
\hline $\mathrm{C}^{34} \mathrm{~S}$ & 1989 & $30 \mathrm{~m}$ & 19 \\
\hline $\mathrm{C}^{18} \mathrm{O} \dagger \ldots \ldots \ldots \ldots \ldots \ldots$ & 1991 & $11 \mathrm{~m} / 30 \mathrm{~m}$ & 20 \\
\hline 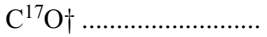 & 1991 & $11 \mathrm{~m} / 30 \mathrm{~m}$ & 20 \\
\hline SO & 1991 & $15 \mathrm{~m}$ & 21 \\
\hline $\mathrm{N}_{2} \mathrm{H}^{+} \dagger \ldots \ldots \ldots \ldots \ldots \ldots \ldots$ & 1991 & $30 \mathrm{~m}$ & 22 \\
\hline $\mathrm{SiO}$ & 1991 & $30 \mathrm{~m}$ & 22 \\
\hline $\mathrm{H}^{13} \mathrm{CO}^{+} \ldots \ldots \ldots$ & 1991 & $30 \mathrm{~m}$ & 22 \\
\hline $\mathrm{HN}^{13} \mathrm{C} \ldots \ldots \ldots \ldots \ldots$ & 1991 & $30 \mathrm{~m}$ & 22 \\
\hline $\mathrm{H}^{13} \mathrm{CN} \dagger \ldots \ldots \ldots \ldots$ & 1991 & $30 \mathrm{~m}$ & 22 \\
\hline $\mathrm{CH}_{3} \mathrm{CCH}$ & 1991 & $30 \mathrm{~m}$ & 23 \\
\hline $\mathrm{CH}_{3} \mathrm{CN}$ & 1991 & $30 \mathrm{~m}$ & 23 \\
\hline${ }^{13} \mathrm{CS}$ & 1993 & $30 \mathrm{~m}$ & 24 \\
\hline OCS & 1995 & $30 \mathrm{~m}$ & 25 \\
\hline 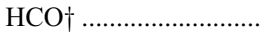 & 1995 & $12 \mathrm{~m}$ & 26 \\
\hline $\mathrm{DCO}^{+} \ldots \ldots \ldots \ldots \ldots \ldots$ & 1996 & $15 \mathrm{~m}$ & 27 \\
\hline DCN & 1996 & $15 \mathrm{~m}$ & 27 \\
\hline $\mathrm{HC}^{15} \mathrm{~N} \dagger \ldots \ldots \ldots \ldots \ldots \ldots$ & 1999 & $15 \mathrm{~m}$ & 28 \\
\hline $\mathrm{H}_{2} \mathrm{~S}$ & 1999 & $15 \mathrm{~m}$ & 29 \\
\hline $\mathrm{H}_{2} \mathrm{CS}$ & 1999 & $15 \mathrm{~m} / 30 \mathrm{~m}$ & 29,30 \\
\hline $\mathrm{CO}^{+} \dagger \ldots \ldots \ldots \ldots \ldots \ldots \ldots \ldots$ & 2000 & $30 \mathrm{~m}$ & 31 \\
\hline 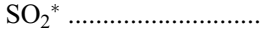 & 2003 & $30 \mathrm{~m}$ & 32 \\
\hline $\mathrm{NO}^{*}$ & 2003 & $30 \mathrm{~m}$ & 32 \\
\hline $\mathrm{NS}^{*}$ & 2003 & $30 \mathrm{~m}$ & 32 \\
\hline${ }^{34} \mathrm{SO}^{*} \ldots \ldots \ldots$. & 2003 & $30 \mathrm{~m}$ & 32 \\
\hline $\mathrm{HOC}^{+} \dagger \ldots \ldots \ldots \ldots \ldots \ldots \ldots \ldots$ & 2004 & $30 \mathrm{~m}$ & 33 \\
\hline 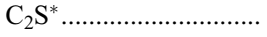 & 2006 & $30 \mathrm{~m}$ & 34 \\
\hline
\end{tabular}

confined. In the case of a source size larger than $20^{\prime \prime}$, column densities would be overestimated by only $\sim 20 \%$, while for sizes down to $10^{\prime \prime}$, the column density would be underestimated by less than a factor of 2. Only in the case of an extremely clumped and compact emission where source sizes were well below $10^{\prime \prime}$, column densities would be dramatically affected. We have also considered the effect of source size on the derived rotation temperature from rotation diagrams. We will assume a case in which we derive $T_{\text {rot }}=15 \mathrm{~K}$ (for a $20^{\prime \prime}$ source) by observing two transitions of a given species at 129 and $175 \mathrm{GHz}$, respectively. In the lower panel in Figure 5 we have plotted the relative change in the derived $T_{\text {rot }}$ for those cases in which the transitions would have upper levels with an energy difference of 60,30 , and $15 \mathrm{~K}$. It is obvious that the more affected $T_{\text {rot }}$ is that derived from the data with the smaller dynamical range in energies. From Figure 5
TABLE 3-Continued

\begin{tabular}{|c|c|c|c|}
\hline Molecule & $\begin{array}{c}\text { Publication } \\
\text { Year }\end{array}$ & Telescope & Reference \\
\hline $\mathrm{CH}_{2} \mathrm{NH}^{*}$. & 2006 & $30 \mathrm{~m}$ & 34 \\
\hline $\mathrm{NH}_{2} \mathrm{CN}^{*}$. & 2006 & $30 \mathrm{~m}$ & 34 \\
\hline $\mathrm{HOCO}^{+*} \ldots \ldots \ldots \ldots \ldots \ldots \ldots$ & 2006 & $30 \mathrm{~m}$ & 34 \\
\hline $\mathrm{C}_{3} \mathrm{H}^{*} \ldots \ldots \ldots \ldots$ & 2006 & $30 \mathrm{~m}$ & 34 \\
\hline $\mathrm{HC}^{18} \mathrm{O}^{+*}$ & 2006 & $30 \mathrm{~m}$ & 34 \\
\hline
\end{tabular}

Notes.-Table adapted and updated from Mauersberger \& Henkel (1993). Asterisks $\left({ }^{*}\right)$ denote molecular species not previously identified outside the Milky Way. Dagger symbols $(\dagger)$ denote species not measured in this survey. Telescopes: (OVRO) Owens Valley interferometer; $(64 \mathrm{~m})$ Parkes $64 \mathrm{~m}$; (11 m) NRAO Kitt Peak $11 \mathrm{~m} ;(100 \mathrm{~m})$ Effelsberg $100 \mathrm{~m}$; (2.3 m) Steward observatory $2.3 \mathrm{~m}$; (7 m) AT\&T Bell Labs 7 m; (43 m) NRAO Green Bank 43 m; (1.4 m) ESO 1.4 m Coudé; (30 m) IRAM Pico Veleta $30 \mathrm{~m}$; $(15 \mathrm{~m})$ ESO SEST $15 \mathrm{~m}$;.

REFERENCES.-(1) Weliachew 1971; (2) Gardner \& Whiteoak 1974; (3) Rickard et al. 1975; (4) Solomon \& de Zafra 1975; (5) Churchwell et al. 1977; (6) Rickard et al. 1977; (7) Thompson et al. 1978; (8) Martin \& Ho 1979; (9) Stark \& Wolff 1979; (10) Whiteoak et al. 1980; (11) Henkel \& Bally 1985; (12) Seaquist \& Bell 1986; (13) Magain \& Gillet 1987; (14) Henkel et al. 1987; (15) Henkel et al. 1988; (16) Mauersberger et al. 1990; (17) Nguyen-Q-Rieu et al. 1989; (18) NguyenQ-Rieu et al. 1991; (19) Mauersberger \& Henkel 1989; (20) Sage et al. 1991; (21) Johansson 1991; (22) Mauersberger \& Henkel 1991; (23) Mauersberger et al. 1991; (24) Henkel et al. 1993; (25) Mauersberger et al. 1995; (26) Sage \& Ziurys 1995; (27) Chin et al. 1996; (28) Chin et al. 1999; (29) Heikkilä et al. 1999; (30) Martín et al. 2005; (31) Fuente et al. 2000; (32) Martín et al. 2003; (33) Usero et al. 2004; (34) this work.

we see that the largest expected uncertainty in the determination of $T_{\text {rot }}$ is smaller than $20 \%$.

\section{DISCUSSION}

\subsection{Extragalactic Molecular Census}

Table 3 lists the detection of all the species known to date in the extragalactic ISM in chronological order. During the last three decades, 29 molecules and 11 rare isotopic substitutions have been detected outside the Galaxy. As a result of the frequency scan presented in this paper (see also Martín et al. 2003, 2005), 8 new molecules and 2 isotopes (denoted by asterisks in Table 3) have been added to the census of known extragalactic species, which represents a $\sim 30 \%$ increase in the total number of identified molecules. While $\mathrm{H}_{2} \mathrm{~S}$ and $\mathrm{H}_{2} \mathrm{CS}$ were detected toward the nearby Large Magellanic Cloud (Heikkilä et al. 1999), their identification in our survey (see Martín et al. 2005) represents the first detections toward a starburst galaxy. Note that $\mathrm{CH}_{2} \mathrm{NH}$, $\mathrm{HOCO}^{+}$, and $\mathrm{C}_{3} \mathrm{H}$ (Table 3 ) are tentatively detected and need confirmation by observations of additional transitions. In Table 3 the known extragalactic species not detected in the $2 \mathrm{~mm}$ band are indicated by dagger symbols.

\subsection{Deuterated Molecules in NGC 253}

The observation of deuterated molecules in external galaxies is interesting for several reasons. The interstellar $\mathrm{D} / \mathrm{H}$ ratio depends on the degree of processing of the gas, since $\mathrm{D}$ was mainly produced soon after the big bang, while nuclear processes during stellar evolution rather destroy than form deuterium. A low $\mathrm{D} / \mathrm{H}$ ratio may therefore indicate that the observed gas component has been recycled in stars, and a higher ratio could be a hint for recent infall of poorly processed material. It has been observed that $D$ is strongly enriched (by a factor of $>10^{4}$ compared to the cosmic $\mathrm{D} / \mathrm{H}$ ratio) in several molecules observable at centimeter and millimeter wavelengths. From model calculations, such a high degree of fractionation is expected if the molecules are being formed in a relatively cool $\left(T_{\text {kin }}<30 \mathrm{~K}\right)$ environment (e.g., 
TABLE 4

Parameters of Selected Deuterated Species

\begin{tabular}{cccccr}
\hline \hline Molecule & $\begin{array}{c}\text { Transition } \\
J-J^{\prime}\end{array}$ & $\begin{array}{c}\nu \\
(\mathrm{MHz})\end{array}$ & $\begin{array}{c}\mathrm{rms}^{\mathrm{a}} \\
(\mathrm{mK})\end{array}$ & $\begin{array}{c}\int T_{\mathrm{MB}} d v^{\mathrm{b}} \\
\left(\mathrm{K} \mathrm{km} \mathrm{s}^{-1}\right)\end{array}$ & {$[\mathrm{DX}] /[\mathrm{HX}]$} \\
\hline $\mathrm{DCO}^{+} \ldots \ldots \ldots \ldots \ldots$ & $2-1$ & 144077.3 & 1.9 & $<0.30$ & $<4 \times 10^{-3 \mathrm{c}}$ \\
$\mathrm{DCN} \ldots \ldots \ldots \ldots \ldots$. & $2-1$ & 144827.9 & 1.1 & $<0.18$ & $<1 \times 10^{-3 \mathrm{c}}$ \\
$\mathrm{DNC} \ldots \ldots \ldots \ldots \ldots$. & $2-1$ & 152609.7 & 3.1 & 0.49 & $\leq 1 \times 10^{-2 \mathrm{c}}$ \\
$\mathrm{N}_{2} \mathrm{D}^{+} \ldots \ldots \ldots \ldots \ldots$. & $2-1$ & 154217.0 & 2.8 & 0.57 & $\leq 1.4 \times 10^{-3 \mathrm{~d}}$ \\
\hline
\end{tabular}

Note.-See $\S 4.2$ for details.

${ }^{a}$ rms computed for $20 \mathrm{~km} \mathrm{~s}^{-1}$ wide channels.

b $3 \sigma$ limit to the integrated intensity assuming a line width of $150 \mathrm{~km} \mathrm{~s}^{-1}$. DNC and $\mathrm{N}_{2} \mathrm{D}^{+}$detections are tentative.

${ }^{c}$ Using the ${ }^{13} \mathrm{C}$ bearing species measured in this survey and assuming ${ }^{12} \mathrm{C} /{ }^{13} \mathrm{C} \sim 40$ (Henkel et al. 1993).

${ }^{\mathrm{d}}$ Using the $J=1-0 \mathrm{~N}_{2} \mathrm{H}^{+}$transition from Mauersberger \& Henkel (1991).

Millar 2002 and references therein). The fact that a high enrichment of deuterated molecules is also observed in hot molecular cores can be explained by evaporation of molecules from grain mantles (e.g., Pineau des Forêts et al. 1989). While a value of $(3 \pm 0.4) \times 10^{-5}$ has been estimated for the primordial $\mathrm{D} / \mathrm{H}$ ratio (Burles 2002 and references therein), a lower ratio of (1.4 \pm $0.24) \times 10^{-5}$ is found in the Galactic neighborhood, and variations are shown on larger scales (Vidal-Madjar 2002). In molecular clouds close to the center of the Milky Way, Jacq et al. (1999) find $\mathrm{DCN}$ and $\mathrm{DCO}^{+}$enhancements that are compatible with $\mathrm{D} / \mathrm{H}$ an order of magnitude smaller than toward the local interstellar medium. This indicates that the gas in the Galactic center region is highly processed. The enhancement of deuterated molecules in the Large Magellanic Cloud, on the other hand, is compatible with the local value of the $\mathrm{D} / \mathrm{H}$ ratio (Chin et al. 1996). No deuterated molecules have been detected so far toward the central regions of other galaxies. Mauersberger et al. (1995) give an upper limit for the DCN/HCN abundance ratio of $4 \times 10^{-3}$ toward NGC 253.

Our survey includes the $J=2-1$ lines of $\mathrm{DCO}^{+}, \mathrm{DCN}, \mathrm{DNC}$, and $\mathrm{N}_{2} \mathrm{D}^{+}$. All other $2 \mathrm{~mm}$ lines of deuterated molecules are expected to be much weaker. Table 4 shows the frequencies of these lines, the rms noise and limits or values of the integrated intensities. $\mathrm{DCO}^{+}$and DCN are not detected. There is a tentative feature at the frequency of DNC and a more significant feature at the line frequency of $\mathrm{N}_{2} \mathrm{D}^{+}$. At the frequencies of $\mathrm{DNC} J=2-1$ no line from another molecule is expected to be strong enough to explain the observed emission. Close to the frequencies of $\mathrm{N}_{2} \mathrm{D}^{+}$ $J=2-1$ is the transition frequency of $\mathrm{OC}^{34} \mathrm{~S} J=13-12$ (at 154.242 GHz). However, from our detections of OCS and our estimates of the ${ }^{32} \mathrm{~S} /{ }^{34} \mathrm{~S}$ ratio (Martín et al. 2005), this emission is expected to be an order of magnitude weaker than what is observed. Another potentially blending line is the $7_{1,6}-6_{1,5}, v_{6}=1$ transition of vibrationally excited $\mathrm{HNCO}$ (at $154.228 \mathrm{GHz}$ ). This line has been detected toward the Galactic hot core G10.47+0.03 with an intensity of about $1 / 5$ that of the corresponding HNCO ground state transition, compatible with a kinetic temperature of about $380 \mathrm{~K}$ (Wyrowski et al. 1999). The feature we are observing here is about as strong as the HNCO $7_{1,6}-6_{1,5}$ ground vibrational state transition (Table 1). If the feature at $154.228 \mathrm{GHz}$ comes from vibrationally excited $\mathrm{HNCO}$, this would either indicate a higher kinetic temperature or maser emission. Both would be remarkable in view of the large volume observed in our beam toward NGC 253. While all this suggests that we have detected, for the first time, one or two deuterated molecules beyond the Magellanic Clouds, we will nevertheless regard the measured features as tentative.
TABLE 5

Limits to the Detection of $\mathrm{C}_{2} \mathrm{H}_{5} \mathrm{OH}$

\begin{tabular}{|c|c|c|c|}
\hline $\begin{array}{c}\nu \\
(\mathrm{MHz})\end{array}$ & $\begin{array}{c}E_{u} \\
\left(\mathrm{~cm}^{-1}\right)\end{array}$ & $\begin{array}{l}\mathrm{rms}^{\mathrm{a}} \\
(\mathrm{mK})\end{array}$ & $\underset{\left(\mathrm{K} \mathrm{km} \mathrm{s}^{-1}\right)}{\int T_{\mathrm{MB}} d v^{\mathrm{b}}}$ \\
\hline $129665.7 \ldots$ & 16.6 & 3.0 & $<1.440$ \\
\hline 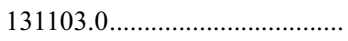 & 16.6 & 1.8 & $<0.864$ \\
\hline 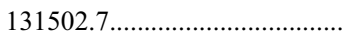 & 20.1 & & $\mathrm{C}_{2} \mathrm{~S}$ \\
\hline 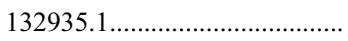 & 6.9 & & Baseline \\
\hline 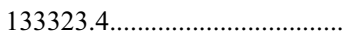 & 16.5 & & $\mathrm{CH}_{2} \mathrm{NH}$ \\
\hline 142285.0 & 25.8 & 1.8 & $<0.864$ \\
\hline 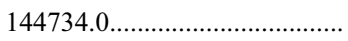 & 9.3 & & $\mathrm{C}^{34} \mathrm{~S}$ \\
\hline 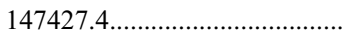 & 21.0 & 2.9 & $<1.392$ \\
\hline $152656.8 \ldots \ldots \ldots \ldots \ldots \ldots \ldots$ & 9.3 & 3.4 & $<1.632$ \\
\hline 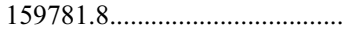 & 12.2 & & $\mathrm{NH}_{2} \mathrm{CN}$ \\
\hline 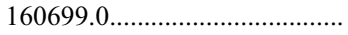 & 31.4 & & $i=\mathrm{H}_{2} \mathrm{~S}$ \\
\hline 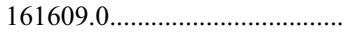 & 26.1 & & NS \\
\hline $173391.3 \ldots \ldots \ldots \ldots$ & 12.2 & 3.6 & $<1.728$ \\
\hline 174232.9 & 15.7 & & $c-\mathrm{C}_{3} \mathrm{H}$ \\
\hline
\end{tabular}

${ }^{a}$ rms computed for $20 \mathrm{~km} \mathrm{~s}^{-1}$ wide channels. If the transition is blended, the species is indicated.

b $3 \sigma$ limit to the integrated intensity assuming an emission width of $150 \mathrm{~km} \mathrm{~s}^{-1}$.

Table 4 gives limits to the $[\mathrm{DX}] /[\mathrm{HX}]$ column density ratios for $\mathrm{HCO}^{+}, \mathrm{HCN}$, and $\mathrm{HNC}$ based on our limits for the $J=2-1$ transitions of the deuterated isotopomers, the integrated intensities of the ${ }^{13} \mathrm{C}$ bearing isotopomers measured in this survey, and the ${ }^{12} \mathrm{C} /{ }^{13} \mathrm{C}$ ratio of 40 obtained by Henkel et al. (1993). We have assumed that the excitation conditions of the deuterated and main species are similar. Intensities were corrected to account for beam dilution assuming a source size of $20^{\prime \prime}$ (see also $\S 3.3$ ). For $\mathrm{N}_{2} \mathrm{D}^{+}$, we have only the $\mathrm{N}_{2} \mathrm{H}^{+} J=1-0$ line for a comparison (Mauersberger \& Henkel 1991). The value for the $\mathrm{N}_{2} \mathrm{D}^{+} / \mathrm{N}_{2} \mathrm{H}^{+}$ column density ratio given in Table 4 assumes optically thin emission, making the same assumptions on the excitation as they have been made for DCN in the Appendix of Mauersberger et al. (1995).

We obtain limits to the $[\mathrm{DX}] /[\mathrm{HX}]$ values of $4 \times 10^{-3}$ for $\mathrm{HCO}^{+}$, of $1 \times 10^{-3}$ for $\mathrm{HCN}$, and $11 \times 10^{-3}$ for HNC. These limits are 2 orders of magnitude above the local interstellar $\mathrm{D} / \mathrm{H}$ ratio and 1 order of magnitude larger than the $[\mathrm{DCN}] /[\mathrm{HCN}]$ ratios estimated for Sgr B2 (Jacq et al. 1999), but 1 order of magnitude lower than the values determined by Chin et al. (1996) in the Large Magellanic Cloud. The limits are consistent with the high kinetic temperatures determined toward the molecular interstellar medium of NGC 253. We can exclude the scenario found in many Galactic hot cores, where recent grain mantle evaporation leads to high $\mathrm{D} / \mathrm{H}$ ratios that were preserved from a time when the temperature of the gas was much lower than it is now.

\subsection{Alcohols in NGC 253}

In the nuclear region of the Milky Way widespread large abundances of the methanol $\left(\mathrm{CH}_{3} \mathrm{OH}\right)$ and ethanol $\left(\mathrm{C}_{2} \mathrm{H}_{5} \mathrm{OH}\right)$ alcohols have been reported (Requena-Torres et al. 2005; Martín-Pintado et al. 2001). This fact has motivated a careful search for the presence of $\mathrm{C}_{2} \mathrm{H}_{5} \mathrm{OH}$ in the $2 \mathrm{~mm}$ scan. Table 5 shows the frequencies and energies of its 14 brightest $2 \mathrm{~mm}$ transitions assuming $T_{\text {rot }}=$ $12 \mathrm{~K}$, as that derived for methanol (see Table 2). We have computed the rms of the noise for $20 \mathrm{~km} \mathrm{~s}^{-1}$ velocity resolution whenever the transition appeared not to be contaminated by other spectral features. From this rms, we estimate a $3 \sigma$ detection limit to the integrated intensity of the transitions. With the limit to the detection of the $152 \mathrm{GHz}$ transition we derive an upper limit 
to the ethanol column density of $N\left(\mathrm{C}_{2} \mathrm{H}_{5} \mathrm{OH}\right)<1.8 \times 10^{14} \mathrm{~cm}^{-2}$. This limit to the column density can be improved if we add up all the transitions that appear not to be blended. We estimate a total column density limit of $N\left(\mathrm{C}_{2} \mathrm{H}_{5} \mathrm{OH}\right)<9.6 \times 10^{13} \mathrm{~cm}^{-2}$.

The methanol-to-ethanol abundance ratio derived for NGC 253 is $\geq 10$. This lower limit is still a factor of 2 below the ratios derived for the GC clouds by Requena-Torres et al. (2005). Thus, further high-sensitivity observations are required to establish if the methanol-to-ethanol abundance ratio is similar to that found in the GC clouds, as expected if alcohols are also ejected from icy grain mantles by shocks in this galaxy (Charnley et al. 1995).

\subsection{The Nuclear Environment in NGC 253}

The detection of new molecules in the extragalactic ISM, far from being a mere census of known species, provides a complete description of the chemical complexity within the nuclear environment of galaxies and a tool to establish the origin of this rich chemistry and the heating of the molecular gas. In the following subsections we will compare the observed abundances in NGC 253 with those available for five external galaxies $(\S 4.4 .1)$ and five prototypical sources within the Milky Way ( $\S 4.4 .2$ ). The comparison focuses mainly on the abundance ratios between the different molecular species compared to those observed toward NGC 253. This will minimize the systematic error in the absolute fractional abundances due to the $\mathrm{H}_{2}$ column density determinations. The relative abundances between molecules will provide us with a reliable chemical differentiation giving us insights into the dominant chemical mechanism acting in each source.

\subsubsection{Comparison with Other Galaxies}

We have selected the nearby galaxies NGC 4945, M82, and IC 342 , where most of the known molecular species have also been observed, and Maffei 2 and NGC 6946, for which fewer species have been detected.

Table 6 presents the integrated intensities of the ${ }^{13} \mathrm{CO} J=$ 2-1 transition that has been used to estimate the $\mathrm{H}_{2}$ sourceaveraged column density. We used the conversion $N\left(\mathrm{H}_{2}\right)=3.3 \times$ $10^{20} \mathrm{~cm}^{-2} I\left({ }^{13} \mathrm{CO}_{2-1}\right)$ (Mauersberger et al. 2003).

Table 7 shows the derived fractional abundances relative to $\mathrm{H}_{2}$ toward the selected galaxies. Because of the frequency coverage of our survey, not all the species listed in Table 3 could be measured. Therefore, in Table 7, we have also included data of additional key molecular species from other studies, namely $\mathrm{NH}_{3}$, $\mathrm{N}_{2} \mathrm{H}^{+}$, and $\mathrm{CN} . \mathrm{H}_{2}$ and $\mathrm{CO}$ are not included as they are tracers of the total mass of the molecular material content. Species such as $\mathrm{OH}$ and $\mathrm{H}_{2} \mathrm{O}$ have also been detected toward NGC 253 and many other galaxies. However, $\mathrm{H}_{2} \mathrm{O}$ exhibits maser emission that does not allow us to determine directly the column densities, while $\mathrm{OH}$ shows extremely complex behavior involving a mixture of absorption, thermal and maser emission (e.g., Turner 1985; Ho et al. 1987; Frayer et al. 1998; Bradford et al. 1999; Henkel et al. 2004; Goicoechea et al. 2005). Unfortunately, there is not enough data available on $\mathrm{CH}, \mathrm{CH}^{+}, \mathrm{HCO}, \mathrm{CO}^{+}$, and $\mathrm{HOC}^{+}$for this comparison.

In order to achieve the highest homogeneity in the derived abundances, we have collected all the line profile parameters from the available observations in the literature. From these values we have made our own estimates of the column densities for each molecule and each source with the assumptions used for NGC 253.

We have assumed a source size $\theta_{s}=20^{\prime \prime}$ for all these sources (see, e.g., § 3.3 for NGC 253 and Wang et al. 2004 for NGC
TABLE 6

Molecular Hydrogen Source-averaged Column Density

\begin{tabular}{|c|c|c|c|c|}
\hline Source & $\begin{array}{c}\text { Transition } \\
J-J^{\prime}\end{array}$ & $\begin{array}{c}\int T_{\mathrm{MB}} d v \\
\left(\mathrm{~K} \mathrm{~km} \mathrm{~s}^{-1}\right)\end{array}$ & $\begin{array}{c}\theta_{\text {beam }} \\
(\operatorname{arcsec})\end{array}$ & $\begin{array}{c}N_{\mathrm{H}_{2}} \\
\left(\mathrm{~cm}^{-2}\right)\end{array}$ \\
\hline NGC 253. & ${ }^{13} \mathrm{CO} 2-1$ & $52.4^{\mathrm{a}}$ & 34 & $6.7 \times 10^{22}$ \\
\hline NGC $4945 \ldots \ldots .$. & ${ }^{13} \mathrm{CO} 2-1$ & $81.2^{\mathrm{b}}$ & 23 & $6.4 \times 10^{22}$ \\
\hline M82 .................... & ${ }^{13} \mathrm{CO} 2-1$ & $29.4^{\mathrm{a}}$ & 34 & $3.8 \times 10^{22}$ \\
\hline IC $342 \ldots \ldots \ldots \ldots$ & ${ }^{13} \mathrm{CO} 2-1$ & $17.2^{\mathrm{a}}$ & 34 & $2.2 \times 10^{22}$ \\
\hline Maffei $2 \ldots \ldots \ldots \ldots$ & ${ }^{13} \mathrm{CO} 2-1$ & $13.9^{\mathrm{a}}$ & 34 & $1.8 \times 10^{22}$ \\
\hline NGC $6946 . . . \ldots .$. & ${ }^{13} \mathrm{CO} 2-1$ & $22.2^{\mathrm{c}}$ & 21 & $1.5 \times 10^{22}$ \\
\hline
\end{tabular}

NotE.-A $20^{\prime \prime}$ source size was assumed for all targets.

a $10 \mathrm{~m}$ HHT by Mauersberger et al. (2003).

b $15 \mathrm{~m}$ SEST by Wang et al. (2004).

c 30 m IRAM by Israel \& Baas (2001).

4945). This is a fairly simple assumption for some of the targets and may lead to considerable errors in the column density determination. As this factor is also included in the source-averaged column density determination of the molecular hydrogen, the error in the resulting fractional abundance will, however, cancel out to first order.

The top panel in Figure 6 shows a graphical representation of the data in Table 7. Molecular species are ordered based on increasing abundances in NGC 253. We have also considered the logarithmic difference of abundances in each selected galaxy with respect to the abundances derived in this work for NGC 253 as $\Delta X=\log _{10}\left(X / X_{\mathrm{NGC} 253}\right)$. This variable, $\Delta X$, is plotted in the bottom panel of Figure 6 . The difference in the $Y$-axis between two species provides a measure of their abundance ratio relative to that in NGC 253.

M82 shows relative abundances quite similar to those of NGC 253 in many but not all of the observed species. Evident chemical differences between both nuclear starbursts were already analyzed (Mauersberger \& Henkel 1993; Takano et al. 1995, 2002; Wang et al. 2004) and have been interpreted as a difference in the evolutionary stage of both starbursts. Compared to that in NGC 253, the nuclear starburst in M82 represents an evolved state where photodissociation regions (PDRs) dominate the heating of the molecular material (García-Burillo et al. 2002). From our comparison, the high relative abundance of $\mathrm{C}_{3} \mathrm{H}_{2}$, a common PDR tracer, is particularly outstanding. On the other hand, we note remarkably low abundances of $\mathrm{CH}_{3} \mathrm{OH}$ and $\mathrm{SiO}$, which are thought to be ejected from grain mantles into the gas phase (Charnley et al. 1995; Martín-Pintado et al. 1992; Requena-Torres et al. 2005). This suggests that shocks do not play the dominant role within the nuclear region of M82. Molecules such as HNCO, apparently associated with shock chemistry (Zinchenko et al. 2000), also show low relative abundances compared to those in NGC 253. Furthermore, the nuclear environment of M82 is characterized by systematically lower relative abundances of $\mathrm{CH}_{3} \mathrm{CN}, \mathrm{NH}_{3}$, and $\mathrm{N}_{2} \mathrm{H}^{+}$. It is also interesting to note how other species used as PDR tracers, namely $\mathrm{C}^{34} \mathrm{~S}$ and $\mathrm{C}_{2} \mathrm{H}$ (Meier \& Turner 2005), do not show high relative abundances in M82 compared to the other observed species. Moreover, they are a factor of $\sim 2-3$ less abundant than most other the species. If we compare the M82 relative abundances with those of the Orion Bar in $\S$ 4.4.2, we find that all the species with low abundances in M82 show the same behavior in the Orion Bar. This comparison strongly supports the idea of M82 as an evolved nuclear starburst largely dominated by PDRs.

In NGC 4945, relative abundances of all the detected molecular species resemble within a factor of 3 those of NGC 253. 
TABLE 7

Molecular Fractional Abundances toward NGC 253 and Other Galaxies

\begin{tabular}{|c|c|c|c|c|c|c|}
\hline Molecule & NGC 253 & NGC 4945 & M82 & IC 342 & Maffei 2 & NGC 6946 \\
\hline $\mathrm{HN}^{13} \mathrm{C}$ & -10.6 & -9.5 & -9.5 & $\ldots$ & $\ldots$ & $\ldots$ \\
\hline $\mathrm{H}^{13} \mathrm{CO}^{+} \ldots \ldots \ldots$ & -10.4 & -10.0 & -9.9 & $-9.5^{\mathrm{a}}$ & $\ldots$ & $\ldots$ \\
\hline $\mathrm{H}^{13} \mathrm{CN}$ & -9.9 & -9.7 & $<-9.9$ & $<-9.4^{\mathrm{a}}$ & $\ldots$ & $\ldots$ \\
\hline $\mathrm{SiO}$ & -9.9 & $<-9.9$ & $<-9.9$ & $<-9.3^{\mathrm{a}}$ & $<-9.1$ & $\ldots$ \\
\hline $\mathrm{CH}_{3} \mathrm{CN}$ & -9.5 & $\ldots$ & $<-9.7$ & $\ldots$ & $\ldots$ & $\ldots$ \\
\hline $\mathrm{C}^{34} \mathrm{~S}$ & -9.4 & -9.4 & -9.3 & $-9.5^{\mathrm{a}}$ & $\cdots$ & $<-9.0$ \\
\hline$c-\mathrm{C}_{3} \mathrm{H}_{2} \ldots \ldots$ & -9.3 & -8.9 & -8.1 & $\ldots$ & $\ldots$ & $\ldots$ \\
\hline $\mathrm{HC}_{3} \mathrm{~N}$ & -9.2 & -8.8 & -8.7 & -8.6 & $\ldots$ & $\ldots$ \\
\hline $\mathrm{N}_{2} \mathrm{H}^{+} \ldots \ldots \ldots \ldots$ & -9.2 & -9.2 & -9.4 & $-9.6^{\mathrm{a}}$ & -9.7 & -9.6 \\
\hline HNC & -9.0 & -8.6 & -8.8 & -8.7 & -8.7 & -8.8 \\
\hline SO & -8.9 & -8.7 & $<-8.5$ & $<-8.7$ & $\ldots$ & $\ldots$ \\
\hline $\mathrm{HCO}^{+} \ldots \ldots \ldots \ldots \ldots \ldots$ & -8.8 & -8.4 & -8.4 & -8.8 & -8.6 & -8.5 \\
\hline 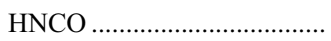 & -8.8 & -8.4 & $<-8.8$ & -8.6 & -8.3 & $\ldots$ \\
\hline $\mathrm{H}_{2} \mathrm{CO}$ & -8.6 & -8.1 & -8.2 & -9.4 & -8.8 & -9.1 \\
\hline OCS & -8.4 & $<-7.5$ & -7.9 & $<-8.6$ & $\ldots$ & $\ldots$ \\
\hline $\mathrm{CN}$ & -8.3 & -7.7 & -8.2 & $-8.3^{\mathrm{a}}$ & $\ldots$ & $\ldots$ \\
\hline $\mathrm{HCN}$ & -8.3 & -8.3 & -8.4 & $-8.5^{\mathrm{a}}$ & -8.3 & -8.5 \\
\hline CS & -8.2 & -8.4 & -8.2 & $-8.4^{\mathrm{a}}$ & -8.7 & -8.7 \\
\hline $\mathrm{CH}_{3} \mathrm{CCH}$ & -8.2 & -8.0 & -7.7 & $\ldots$ & $\ldots$ & $\ldots$ \\
\hline $\mathrm{CH}_{3} \mathrm{OH}$ & -7.9 & -7.4 & $<-8.3$ & $<-7.8$ & -7.7 & -7.9 \\
\hline $\mathrm{C}_{2} \mathrm{H}$ & -7.7 & -7.3 & -7.6 & $<-8.4$ & $\ldots$ & $\ldots$ \\
\hline $\mathrm{NH}_{3} \ldots \ldots$ & -7.2 & $\ldots$ & -8.4 & -7.3 & -7.1 & $\ldots$ \\
\hline
\end{tabular}

a Observations taken $\sim 15^{\prime \prime}$ away from the central position (see $\S 4.4 .1$ for details).

REFERENCES.-For NGC 253: (NS, NO) this paper, Martín et al. 2003; (OCS) Martín et al. 2005; $\left(\mathrm{NH}_{3}\right)$ Mauersberger et al. 2003; (CN) Henkel et al. 1988; $\left(\mathrm{N}_{2} \mathrm{H}^{+}\right)$Mauersberger \& Henkel 1991. For NGC 4945: (SiO) Wang et al. 2004, Henkel et al. 1994. For M82: (CS, $\left.\mathrm{C}^{34} \mathrm{~S}\right)$ Mauersberger \& Henkel 1989; $\left(\mathrm{N}_{2} \mathrm{H}^{+}, \mathrm{SiO}, \mathrm{H}^{13} \mathrm{CO}^{+}\right)$Mauersberger \& Henkel 1991; $\left(\mathrm{C}_{3} \mathrm{H}_{2}, \mathrm{CH}_{3} \mathrm{CN}, \mathrm{CH}_{3} \mathrm{CCH}\right)$ Mauersberger et al. 1991; (OCS) Mauersberger et al. 1995; $\left(\mathrm{H}_{2} \mathrm{CO}, \mathrm{CH}_{3} \mathrm{OH}\right)$ Hüttemeister et al. 1997; $\left(\mathrm{CN}, \mathrm{HC}_{3} \mathrm{~N}, \mathrm{C}_{2} \mathrm{H}\right)$ Henkel et al. 1988; $\left(\mathrm{HCO}^{+}, \mathrm{HCN}, \mathrm{H}^{13} \mathrm{CN}\right)$ Henkel et al. 1998; (SO) Petuchowski \& Bennett 1992; (HCNO) Nguyen-Q-Rieu et al. 1991; $\left(\mathrm{HCN}, \mathrm{HCO}^{+}, \mathrm{H}^{13} \mathrm{CO}^{+}\right)$Nguyen-Q-Rieu et al. 1992; ( $\left.\mathrm{HCN}, \mathrm{HCO}^{+}\right)$Wild 1990; $\left(\mathrm{NH}_{3}\right)$ Wess et al. 2001; ( $\mathrm{HNC}$ ) Hüttemeister et al. 1995. For IC 342: (CS) Mauersberger \& Henkel 1989; $\left(\mathrm{N}_{2} \mathrm{H}^{+}, \mathrm{SiO}, \mathrm{H}^{13} \mathrm{CO}^{+}\right)$Mauersberger \& Henkel $1991 ;\left(\mathrm{C}^{34} \mathrm{~S}, \mathrm{OCS}, \mathrm{HC}_{3} \mathrm{~N}\right.$, $\mathrm{H}_{2} \mathrm{CO}$ ) Mauersberger et al. 1995; $\left(\mathrm{CN}, \mathrm{HNC}, \mathrm{HC}_{3} \mathrm{~N}\right)$ Henkel et al. 1988; ( $\left.\mathrm{HNC}\right)$ Hüttemeister et al. 1995; $\left(\mathrm{H}_{2} \mathrm{CO}, \mathrm{CH}_{3} \mathrm{OH}\right)$ Hüttemeister et al. 1997; (SO) Petuchowski \& Bennett 1992; (HCN, HCO ${ }^{+}$) Wild 1990; (HCNO) Nguyen-Q-Rieu et al. 1991; $\left(\mathrm{HCN}, \mathrm{HCO}^{+}, \mathrm{H}^{13} \mathrm{CO}^{+}\right)$Nguyen-Q-Rieu et al. 1992; $\left(\mathrm{HCN}, \mathrm{H}^{13} \mathrm{CN}\right)$ Henkel et al. 1998; $\left(\mathrm{C}_{2} \mathrm{H}\right)$ Meier \& Turner 2005; $\left(\mathrm{NH}_{3}\right)$ Mauersberger et al. 2003. For Maffei 2: (CS) Mauersberger \& Henkel 1989; $\left(\mathrm{SiO}, \mathrm{N}_{2} \mathrm{H}^{+}\right)$Mauersberger \& Henkel 1991; $\left(\mathrm{H}_{2} \mathrm{CO}\right.$, $\left.\mathrm{CH}_{3} \mathrm{OH}\right)$ Hüttemeister et al. 1997; ( $\left.\mathrm{HCNO}\right)$ Nguyen-Q-Rieu et al. 1991; ( $\left.\mathrm{HCO}^{+}, \mathrm{HCN}\right)$ Nguyen-Q-Rieu et al. 1992; $\left(\mathrm{NH}_{3}\right) \mathrm{Henkel}^{2}$ et al. 2000, Mauersberger et al. 2003. For NGC 6946: $\left(\mathrm{H}_{2} \mathrm{CO}, \mathrm{CH}_{3} \mathrm{OH}\right)$ Hüttemeister et al. 1997; $\left(\mathrm{CS}, \mathrm{C}^{34} \mathrm{~S}\right)$ Mauersberger \& Henkel 1989; ( $\left.\mathrm{HCO}^{+}, \mathrm{HCN}\right)$ Nguyen-Q-Rieu et al. 1992; $\left(\mathrm{N}_{2} \mathrm{H}^{+}\right)$Mauersberger \& Henkel 1991.

The nondetection of $\mathrm{SiO}$ indicates a clear underabundance, similar to what is seen toward M82. On the other hand, unlike in $\mathrm{M} 82, \mathrm{CH}_{3} \mathrm{OH}$ shows an abundance similar to that in NGC 253. This resemblance between the abundances in NGC 4945 and NGC 253, altogether with excitation considerations and a detailed study of species such as $\mathrm{CN}, \mathrm{HCN}$, and $\mathrm{HNC}$, has been interpreted as an intermediate evolutionary state, between NGC 253 and M82 (Wang et al. 2004). From our comparison, the nuclear starburst of NGC 4945 shows much closer resemblance to NGC 253 than to M82.

IC 342 shows high relative abundances of $\mathrm{HC}_{3} \mathrm{~N}_{\text {and }} \mathrm{H}^{13} \mathrm{CO}^{+}$, while molecules such as $\mathrm{C}_{2} \mathrm{H}$ and $\mathrm{N}_{2} \mathrm{H}^{+}$have lower abundances as compared with NGC 253. Many of the available observations for IC 342 (marked by an asterisk in Table 7) were obtained near the $\mathrm{D}$ position as labeled by Downes et al. (1992), $\sim 15^{\prime \prime}$ north of the main nuclear star formation region. The abundance of $\mathrm{C}_{2} \mathrm{H}$ given in Table 7 has been taken from Meier \& Turner (2005) toward the $\mathrm{D}$ position. Its relative abundance is low compared to that in NGC 253. Even taking the $\mathrm{C}_{2} \mathrm{H}$ abundance at the position where this molecule peaks (i.e., a value an order of magnitude higher toward the central position, Meier \& Turner 2005), the relative abundances of this species with respect to the rest of molecules would be similar to that in NGC 253. High-resolution maps from Meier \& Turner (2005) clearly differentiate a region dominated by UV radiation from the central nuclear cluster and the region where the chemistry is dominated by shocks produced by collisions of clouds in a barred potential. Given the position toward which many of the spectra were taken, away from the dynamical center, the molecular emission of IC 342 should resemble more that of NGC 253 than that of M82. In fact IC 342 does not show the relatively low $\mathrm{HNCO}$ abundance seen in M82 but does show a similar low relative abundance of $\mathrm{C}_{2} \mathrm{H}$. Still, there is a large number of molecules not yet observed that are critical to fully evaluate the chemistry of IC 342 , such as $\mathrm{SiO}, \mathrm{SO}$, OCS, and $\mathrm{CH}_{3} \mathrm{OH}$.

As far as Maffei 2 and NGC 6946 are concerned, the available molecular information is not sufficient to establish a clear chemical differentiation from the other sources. What can be noted is a remarkably high relative abundance of $\mathrm{HNCO}$ in Maffei 2 compared to the other galaxies and a low relative abundance of $\mathrm{H}_{2} \mathrm{CO}$ in NGC 6946. Both galaxies also show low relative abundances of $\mathrm{N}_{2} \mathrm{H}^{+}$.

In order to quantify this comparison, Table 8 shows the mean and rms of $\Delta X$ for each source for two cases. The first only uses the species detected in both sources. The number of molecules included in the calculations is also indicated. The second also uses upper limits to the abundances derived for the comparison sources. To take these limits into account, we followed two 

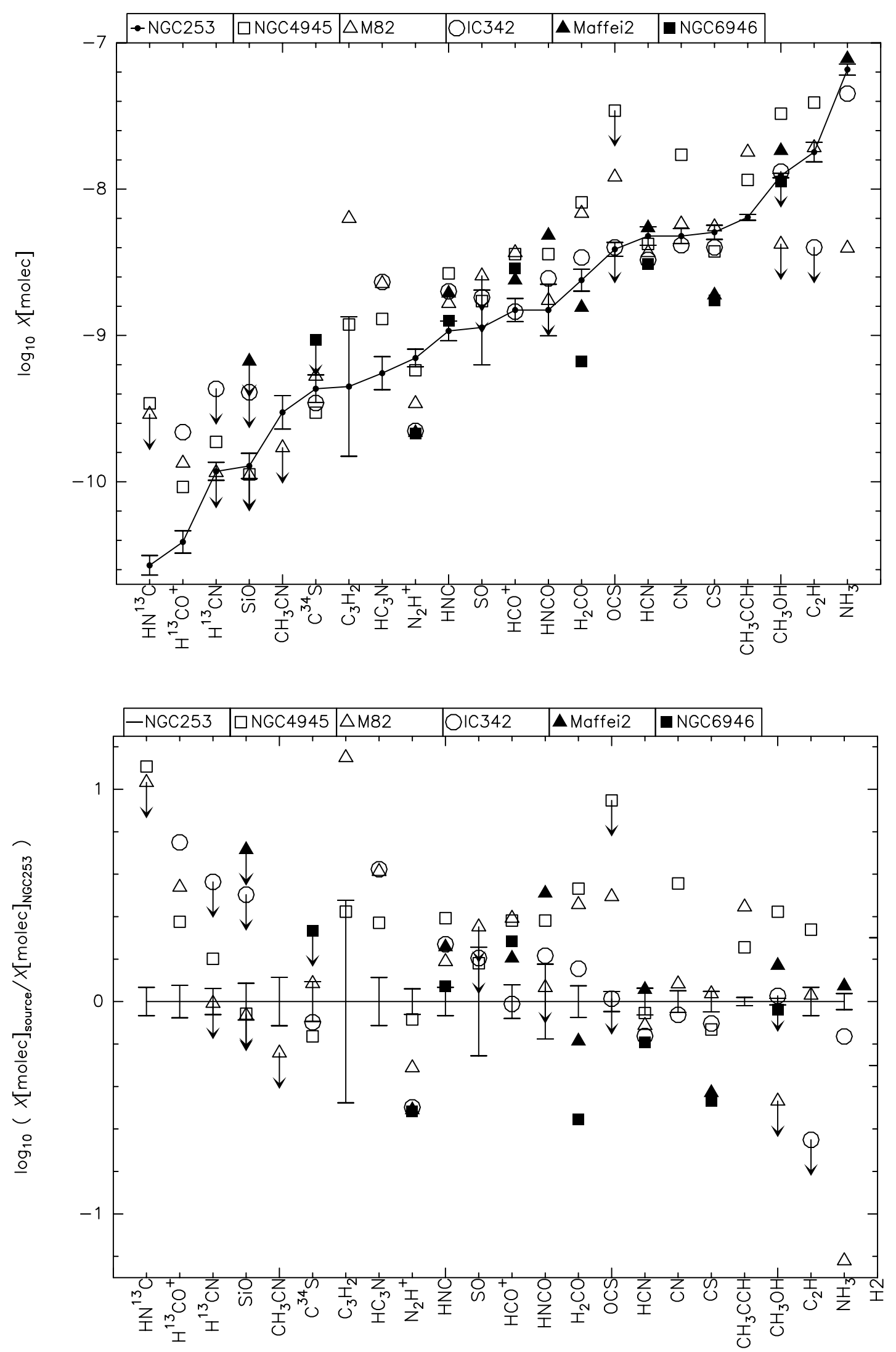

Fig. 6.-Molecular abundances $(X)$ of selected extragalactic sources compared to those of NGC 253. Arrows represent upper limits. A solid line connects the abundances in NGC 253. Top panel: Logarithmic fractional abundances relative to $\mathrm{H}_{2}$ as presented in Table 7. Bottom panel: Logarithmic abundances for each species normalized to that measured in NGC 253 
TABLE 8

Statistical Comparison of Abundances of Selected Galaxies WITh Those IN NGC 253

\begin{tabular}{|c|c|c|c|c|c|c|}
\hline \multirow[b]{2}{*}{ Source } & \multicolumn{3}{|c|}{ Without Limits } & \multicolumn{3}{|c|}{ With LiMITS } \\
\hline & $\overline{\Delta X}$ & $\operatorname{rms}(\Delta X)$ & Number & $\overline{\Delta X}$ & $\operatorname{rms}(\Delta X)$ & Number \\
\hline NGC $4945 \ldots . .$. & 0.30 & 0.29 & 18 & 0.27 & 0.32 & 19 \\
\hline M82 ................... & 0.19 & 0.51 & 15 & 0.05 & 0.54 & 19 \\
\hline IC $342 \ldots \ldots \ldots \ldots . . .$. & 0.08 & 0.34 & 12 & 0.00 & 0.42 & 13 \\
\hline Maffei $2 \ldots \ldots \ldots . .$. & 0.02 & 0.31 & 9 & 0.02 & 0.31 & 9 \\
\hline NGC $6946 \ldots . . .$. & -0.20 & 0.30 & 7 & -0.20 & 0.30 & 7 \\
\hline
\end{tabular}

Notes. $-\Delta X=\left(\log _{10} X / X_{\mathrm{NGC} 253}\right)$. See last paragraph in $\S 4.4 .1$ for details.

criteria: upper limits higher than the abundances measured toward NGC 253 were rejected as they do not provide useful information for the comparison; for the calculations, the other observed limits, lower than the abundances in NGC 253, were divided by 2 as an estimated true value. The second assumption may overestimate some abundances, but any other constraint might result in biased guesses. While the mean value, $\overline{\Delta X}$, only gives an the overall absolute difference in the abundances of each source as compared with NGC 253, the rms of $\Delta X$ provides us with a fairly reliable measurement of differences in the abundance pattern that defines the chemistry of each object. M82, as pointed out before, clearly differs chemically from NGC 253, showing the highest rms values. NGC 4945 and IC 342 are much closer to NGC 253.

\subsubsection{Comparison with Prototype Galactic Sources}

We have selected five Galactic sources, namely Sgr B2(N), Sgr B2(OH), TMC-1, L134N, and the Orion Bar, which are considered to be prototypes of their respective chemistry within the Galaxy. All these sources have been the target of multitransition studies and some of them of frequency surveys. Table 9 shows the abundances of all the molecules observed in the NGC 253 survey compared to those of these Galactic sources. In order to achieve consistency among ratios, we have tried, when available, to use values from line surveys or multiline studies for each source.

In the same way as in the extragalactic comparison, the top panel in Figure 7 shows a graphical representation of the data in Table 9, where molecular species are ordered based on increasing NGC 253 abundances. The bottom panel in Figure 7 shows the logarithmic abundance of each molecule normalized to the abundance measured in NGC 253.

Sgr B2(N) is believed to represent the prototype of a hot molecular core chemistry associated with massive star formation near the nucleus of the Milky Way. A large number of molecular species have been identified toward this source, which is the brightest molecular line emitter within the Galaxy (Friedel et al. 2004 and references therein). There are clear differences between the abundances found toward Sgr B2(N) and those in NGC 253. A set of molecules, namely $\mathrm{CH}_{3} \mathrm{CN}, \mathrm{HC}_{3} \mathrm{~N}, \mathrm{NS}, \mathrm{H}_{2} \mathrm{CS}, \mathrm{SO}_{2}, \mathrm{CH}_{2} \mathrm{NH}, \mathrm{SO}$, $\mathrm{CH}_{3} \mathrm{OH}$, and $\mathrm{NO}$, show abundances relative to the other species $\sim 2-3$ orders of magnitude larger than what we observe toward

TABLE 9

Comparison of NGC 253 Fractional Molecular Abundances with Those in Selected Galactic Sources

\begin{tabular}{|c|c|c|c|c|c|c|}
\hline Molecule & NGC 253 & Sgr B2(N) & Sgr B2(OH) & TMC-1 & L134N & Orion Bar \\
\hline $\mathrm{HN}^{13} \mathrm{C}$. & -10.6 & -11.0 & $\ldots$ & $\ldots$ & $\ldots$ & $\ldots$ \\
\hline 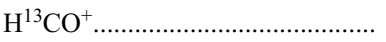 & -10.4 & -11.4 & $\ldots$ & $\ldots$ & $\ldots$ & -10.3 \\
\hline $\mathrm{SiO}$ & -9.9 & -10.7 & $\ldots$ & $<-11.6$ & $<-11.4$ & -10.3 \\
\hline 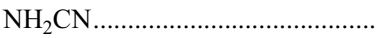 & -9.7 & -10.1 & -10.0 & $\ldots$ & $\ldots$ & $\ldots$ \\
\hline 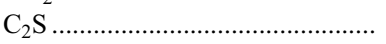 & -9.7 & $\ldots$ & -9.6 & -8.1 & -9.2 & $\ldots$ \\
\hline $\mathrm{CH}_{3} \mathrm{CN}$ & -9.5 & -6.7 & -9.4 & -9.0 & $<-9.0$ & $<-10.3$ \\
\hline 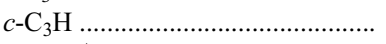 & -9.5 & -10.5 & $<-10.9$ & -9.3 & $\ldots$ & $\ldots$ \\
\hline 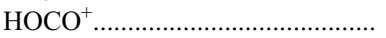 & -9.4 & -10.5 & -9.7 & $\ldots$ & $\ldots$ & $\ldots$ \\
\hline $\mathrm{C}^{34} \mathrm{~S}$ & -9.4 & -10.2 & $\ldots$ & $\ldots$ & $\ldots$ & -9.0 \\
\hline$c-\mathrm{C}_{3} \mathrm{H}_{2} \ldots \ldots \ldots \ldots \ldots \ldots \ldots \ldots \ldots \ldots \ldots \ldots \ldots \ldots \ldots \ldots \ldots \ldots$ & -9.3 & -10.5 & -9.8 & -8.0 & -8.7 & -9.7 \\
\hline 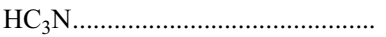 & -9.2 & -7.5 & -9.0 & -8.2 & -9.7 & $\ldots$ \\
\hline 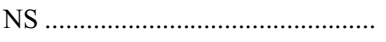 & -9.2 & -7.0 & $\ldots$ & -9.1 & -9.5 & $\ldots$ \\
\hline $\mathrm{H}_{2} \mathrm{CS}$ & -9.2 & -6.8 & -8.7 & -8.5 & -9.2 & $\ldots$ \\
\hline 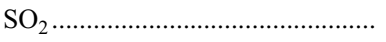 & -9.1 & -6.6 & -8.7 & $<-9.0$ & -8.4 & -9.9 \\
\hline 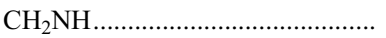 & -9.1 & -7.0 & -9.2 & $\ldots$ & $\ldots$ & $\ldots$ \\
\hline $\mathrm{H}_{2} \mathrm{~S}$ & -9.1 & -9.9 & $\ldots$ & $<-9.3$ & -9.1 & -8.2 \\
\hline 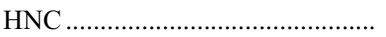 & -9.0 & $\ldots$ & $\ldots$ & -7.7 & -8.2 & -9.0 \\
\hline SO & -8.9 & -6.9 & -8.7 & -8.3 & -7.7 & -8.0 \\
\hline 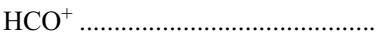 & -8.8 & $\ldots$ & $\ldots$ & -8.1 & -8.1 & -8.5 \\
\hline 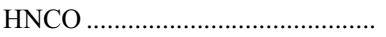 & -8.8 & -9.2 & -8.4 & -9.7 & $\ldots$ & $<-10.8$ \\
\hline $\mathrm{H}_{2} \mathrm{CO}$ & -8.6 & -9.3 & -8.6 & -7.7 & -7.7 & -8.2 \\
\hline OCS & -8.4 & -8.6 & -8.3 & -8.7 & -8.7 & $\ldots$ \\
\hline 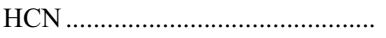 & -8.3 & $\cdots$ & $\ldots$ & -7.7 & -8.4 & -8.3 \\
\hline $\mathrm{CS}$ & -8.2 & $\ldots$ & $\ldots$ & -8.0 & -9.0 & -7.6 \\
\hline $\mathrm{CH}_{3} \mathrm{CCH}$ & -8.2 & -8.4 & -8.8 & -8.2 & $<-8.9$ & $\ldots$ \\
\hline $\mathrm{CH}_{3} \mathrm{OH}$ & -7.9 & -5.8 & -7.3 & -8.7 & -8.5 & -9.0 \\
\hline 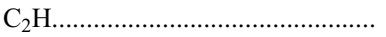 & -7.7 & -9.7 & $\ldots$ & -7.1 & $<-7.3$ & -8.7 \\
\hline 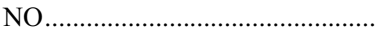 & -7.2 & -6.0 & $\ldots$ & $<-7.5$ & -7.2 & -8.6 \\
\hline
\end{tabular}

REFERENCES.-For NGC 253: (NS, NO) this paper, Martín et al. (2003); (OCS) Martín et al. (2005). For Sgr B2N: (H2S) Nummelin et al. (2000), Minh et al. (1991). $N\left(\mathrm{H}_{2}\right)=3.0 \times 10^{24} \mathrm{~cm}^{-2}$ from Nummelin et al. (2000). For Sgr B2(OH): Cummins et al. (1986), Turner (1991). $N\left(\mathrm{H}_{2}\right)=5 \times 10^{23} \mathrm{~cm}^{-2}$ (see text for details on this estimate). For TMC-1 and L134N: (NS) Ohishi et al. (1992), McGonagle et al. (1994); (SiO) Ziurys et al. (1989). $N\left(\mathrm{H}_{2}\right)=1.0 \times 10^{22} \mathrm{~cm}^{-2}$ from Ohishi et al. (1992). For Orion Bar: $\left(\mathrm{C}_{3} \mathrm{H}_{2}\right)$ Jansen et al. (1995), Fuente et al. (2003); (SiO) Schilke et al. (2001). $N\left(\mathrm{H}_{2}\right)=6.5 \times 10^{22} \mathrm{~cm}^{-2}$ from Jansen et al. (1995). 

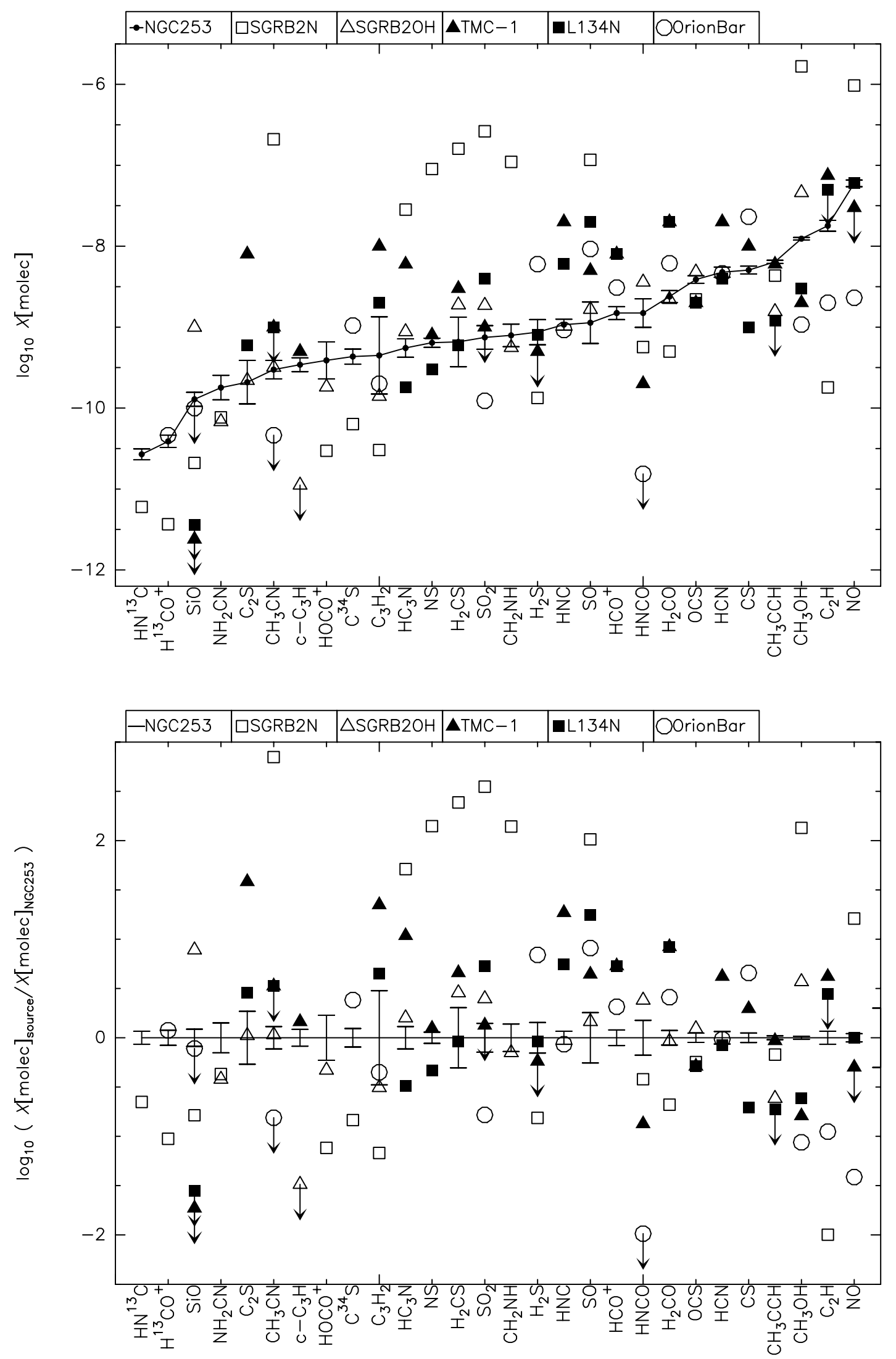

FIG. 7.-Molecular abundances $(X)$ of selected Galactic sources compared to those of NGC 253. Arrows represent upper limits. A solid line connects the abundances in NGC 253. Top panel: Logarithmic fractional abundances relative to $\mathrm{H}_{2}$ as shown in Table 9. Bottom panel: Logarithmic abundances for each species normalized to that measured in NGC 253. 
NGC 253. Almost all the other species observed toward Sgr $\mathrm{B} 2(\mathrm{~N})$ have smaller abundances than in NGC 253, but their relative abundances are, within an order of magnitude, similar to those in $\mathrm{NGC} 253$. Exceptions are $\mathrm{HOCO}^{+}$and $\mathrm{C}_{2} \mathrm{H}$, which show lower relative abundances by factors of $\sim 30$ and 100 , respectively, in the Sgr B2(N) hot core. Moreover, the rotation temperatures derived from the observed molecules in NGC 253 are low compared to the typical temperatures of $>70 \mathrm{~K}$ observed toward hot cores. Even if hot cores associated with massive protostars are present in the nuclear environment of NGC 253, they clearly do not dominate the molecular emission. While the angular size of the Sgr B2 molecular cloud complex is $\sim 18^{\prime}$ (Scoville et al. 1975), the emission of some large complex molecules toward Sgr B2(N) appears concentrated within a region of $\sim 5^{\prime \prime}$, corresponding to $0.2 \mathrm{pc}$ at a distance of $8.5 \mathrm{kpc}$ (Kerr \& Lynden-Bell 1986). At the distance of NGC 253, such a source would have a diameter of $<0$ ". 02 , and because of beam dilution, its detection is certainly out of reach for the $30 \mathrm{~m}$ telescope. A large number of such sources would be needed in order to be able to observe traces of their complex chemistry.

The Orion Bar has been selected as the prototype of photodissociation regions (PDRs). A large fraction of the molecular gas in the Milky Way and in external galaxies is expected to be affected by PDRs (Hollenbach \& Tielens 1997). In PDRs, molecular emission originates from the surface layers of interstellar molecular clouds exposed to intense far-ultraviolet photons from nearby young OB stars. The nondetection of molecules such as $\mathrm{CH}_{3} \mathrm{CN}$ and $\mathrm{HNCO}$ toward the Orion PDR clearly indicates a low relative abundance of these species with respect to other molecules, which is in contrast with the observed NGC 253 abundances. Similarly, the relative abundances of $\mathrm{SiO}, \mathrm{SO}_{2}, \mathrm{CH}_{3} \mathrm{OH}$, $\mathrm{C}_{2} \mathrm{H}$ and $\mathrm{NO}$ appear to be $\sim 1-2$ orders of magnitude lower in the Orion bar than in NGC 253. High abundances of hydrocarbon radicals, such as $\mathrm{C}_{2} \mathrm{H}$ and $\mathrm{C}_{3} \mathrm{H}_{2}$, are observed toward PDRs (Fossé et al. 2000; Fuente et al. 1993, 2003; Pety et al. 2005). In this context, it is surprising to see how $\mathrm{C}_{2} \mathrm{H}$ and $\mathrm{C}_{3} \mathrm{H}_{2}$, commonly used as PDR tracers, show relatively low abundances in the Orion PDR when compared not only with NGC 253 but also with the prototypical Galactic sources except the Sgr B2(N) hot core. The observations of the Orion Bar have been taken from the position of maximum $\mathrm{H}_{2}$ column density (Jansen et al. 1995; Fuente et al. 1996), which does not match the position of highest $\mathrm{C}_{3} \mathrm{H}_{2}$ abundance (Fuente et al. 2003). However, the contribution of the position of the $\mathrm{C}_{3} \mathrm{H}_{2}$ emission peak to the total column density is smaller than that of the $\mathrm{H}_{2}$ column density peak. Therefore, it is expected that the molecular abundance of the $\mathrm{H}_{2}$ peak dominates the emission of PDRs, also in external galaxies. Anyhow, even if we consider the position of the larger $\mathrm{C}_{3} \mathrm{H}_{2}$ abundance (up to 1 order of magnitude; Fuente et al. 2003), the relative abundance would still be close to that found in NGC 253. From this comparison as well as that to M82 (see $\S 4.4 .1)$ it is clear that the chemistry of the nuclear environment of NGC 253 is not dominated by photodissociation.

Quiescent cold dark clouds are represented by TMC1 and L134N. The molecular composition at sites of low-mass star formation is dominated by gas-phase ion-molecule chemistry due to a lack of embedded luminous sources. In our comparison, both dark cloud complexes have in common low relative abundances of $\mathrm{SiO}$ and, to a lesser degree, of $\mathrm{CH}_{3} \mathrm{OH}$. The remaining species behave quite differently in each source. $\mathrm{SO}_{2}, \mathrm{H}_{2} \mathrm{~S}, \mathrm{HNCO}$, and $\mathrm{NO}$ show low abundances in TMC-1, while $\mathrm{C}_{2} \mathrm{~S}$ and $\mathrm{C}_{3} \mathrm{H}_{2}$ appear to have abundances larger than in NGC 253 by up to 1 order of magnitude. On the other hand, L134N shows a high
TABLE 10

Statistical Comparison of Abundances of Selected Galactic Sources with Those IN NGC 253

\begin{tabular}{|c|c|c|c|c|c|c|}
\hline \multirow[b]{2}{*}{ Source } & \multicolumn{3}{|c|}{ Without Limits } & \multicolumn{3}{|c|}{ With Limits } \\
\hline & $\overline{\Delta X}$ & $\operatorname{rms}(\Delta X)$ & Number & $\overline{\Delta X}$ & $\operatorname{rms}(\Delta X)$ & Number \\
\hline Sgr $B 2(N) \ldots \ldots .$. & 0.40 & 1.50 & 22 & 0.40 & 1.50 & 22 \\
\hline Sgr B2 $(\mathrm{OH}) \ldots .$. & 0.07 & 0.40 & 16 & -0.04 & 0.58 & 17 \\
\hline TMC-1............. & 0.47 & 0.66 & 18 & 0.26 & 0.85 & 21 \\
\hline L134N ............... & 0.18 & 0.58 & 16 & -0.00 & 0.76 & 18 \\
\hline Orion Bar .......... & -0.07 & 0.71 & 14 & -0.28 & 0.85 & 17 \\
\hline
\end{tabular}

NotE.-See Table 8 and $\S 4.4 .2$ for details.

SO abundance and relatively low abundances of $\mathrm{HC}_{3} \mathrm{~N}$ and $\mathrm{CH}_{3} \mathrm{CCH}$. The abundance pattern defined by these two dark clouds clearly does not follow the relative abundances found toward NGC 253. In addition, even the rotation temperatures derived for NGC 253, which in general are smaller than $T_{\text {kin }}$, are higher than the typical kinetic temperature $\left(T_{\text {kin }} \sim 10 \mathrm{~K}\right)$ measured toward dark clouds.

Sgr B2(OH), located at the southern end of the Sgr B2 molecular envelope, is free from the emission stemming from the hot cores in this cloud. This position in the Sgr B giant molecular cloud is taken as the prototype of Galactic center (GC) molecular cloud complexes (Martín-Pintado et al. 1997). The $\mathrm{H}_{2}$ column density of this source has been estimated to be roughly $N\left(\mathrm{H}_{2}\right)=$ $5 \times 10^{23}$ (Nummelin et al. 2000). The relative abundances of all the species measured toward this Galactic center molecular cloud complex appear to closely follow the abundance pattern in NGC 253 within a factor $\sim 5$. The only species clearly underabundant in Sgr B2 $(\mathrm{OH})$ when compared to NGC 253 is $c-\mathrm{C}_{3} \mathrm{H}$, but this molecule is only tentatively detected toward NGC 253 . Thus, the ratios between any of the observed species are similar to those in NGC 253 within 1 order of magnitude.

In analogy to our statistical comparison of molecular abundances in prototypical nearby galaxies shown in Table 8, Table 10 presents a comparison between NGC 253 and the selected Galactic sources. To calculate the parameters, the available abundances in Table 9 have been taken into account following the criteria explained at the end of $\S$ 4.4.1. Given the uncertainty of its detection, $c-\mathrm{C}_{3} \mathrm{H}$ was not included. The computed rms of the logarithmic differences shows the envelope of the Sgr B2 complex as the one most closely resembling NGC 253.

The agreement in relative abundances between NGC 253 and a typical Galactic center cloud is a remarkable and clear indication that the heating and chemistry of the bulk of the ISM is dominated by the same processes. The molecular envelope around the star forming region Sgr B2 is formed by a relatively dense and warm material $\left(n_{\mathrm{H}_{2}}=2 \times 10^{5} \mathrm{~cm}^{-3}\right.$ and $T_{\text {kin }}=40-60 \mathrm{~K}$; de Vicente et al. 1997). The large abundances of $\mathrm{NH}_{3}, \mathrm{SiO}$, and $\mathrm{C}_{2} \mathrm{H}_{5} \mathrm{OH}$ observed in the gas phase of the Galactic center molecular clouds are claimed to be the result of grain chemistry and subsequent ejection onto the gas phase due to the disruption of grains by low-velocity shocks $\left(v<20 \mathrm{~km} \mathrm{~s}^{-1}\right.$, Flower et al. 1995; Martín-Pintado et al. 1997, 2001). In the Galactic center, the possible origin for these shocks is so far unclear. It has been claimed that they could be produced by the large-scale shocks associated with cloud-cloud collisions due to the orbital motion of molecular clouds in a barred potential (Hasegawa et al. 1994; Sato et al. 2000) and the interaction of expanding bubbles due to supernova events and/or strong stellar winds from Wolf-Rayet 
stars (Sofue 1990; de Vicente et al. 1997; Martín-Pintado et al. 1999) associated with a burst of star formation that occurred $10^{7} \mathrm{yr}$ ago (Rodríguez-Fernández et al. 2004; Rodríguez-Fernández \& Martín-Pintado 2005).

\section{CONCLUSIONS}

1. We present the first unbiased molecular line survey of an extragalactic source. The survey covers the $2 \mathrm{~mm}$ atmospheric window from 129.1 to $175.2 \mathrm{GHz}$ toward the inner $200 \mathrm{pc}$ of NGC 253. A total of 111 features are identified as transitions of 25 different molecular species, eight of which (three tentatively) have been detected for the first time outside the Milky Way. The rare isotopes, ${ }^{34} \mathrm{SO}$ and $\mathrm{HC}^{18} \mathrm{O}^{+}$, were also detected for the first time in an extragalactic source. In addition, three hydrogen recombination lines and, tentatively, two deuterated species are identified, $\mathrm{N}_{2} \mathrm{D}^{+}$, and DNC. The origin of the observed features of both $\mathrm{N}_{2} \mathrm{D}^{+}$, and $\mathrm{DNC}$ is still unclear and deserves further investigation. If real, these would represent the first deuterated molecules observed beyond the Magellanic Clouds. Column densities and rotation temperatures have been determined for each species under local thermodynamic equilibrium (LTE) conditions.

2. As a result of this survey and by adding existing data from three molecules, namely $\mathrm{NH}_{3}, \mathrm{~N}_{2} \mathrm{H}^{+}$, and $\mathrm{CN}$, we obtain the most complete description to date of the chemical complexity within the nuclear few hundred parsecs of a starburst galaxy. We have performed a comparison between the chemistry of NGC 253 and those of five other outstanding nearby galaxies. This comparison clearly shows the strong chemical differentiation between nuclei of galaxies. Most prominent differences are observed between NGC 253 and M82. This can be interpreted in terms of a more evolved stage of the nuclear starburst in M82. The chemistry of NGC 4945, although claimed to be at an intermediate stage of evolution between NGC 253 and M82, clearly resembles more that of NGC 253 except for the lack of SiO.
Similarly, the position within the nuclear region of IC 342, where most of the molecular observations have been made, show relative abundances close to those in NGC 253. This position is far from the main region of star formation where PDRs are claimed to dominate the chemistry.

As far as Maffei 2 and NGC 6946 are concerned, the available molecular observations do not yet allow us to carry out a similarly detailed comparison.

3. A comparison of the molecular abundances of NGC 253 with selected Galactic sources shows a striking similarity between NGC 253 and Sgr B2(OH). The latter source, located in the envelope of Sgr B2, is commonly taken as the prototype of the molecular cloud complexes in the Galactic center region. This indicates that the chemistry of the nuclear molecular environment in NGC 253 and the Galactic center molecular clouds are driven by similar chemical processes. If this is the case, the chemistry and the heating of the nuclear molecular material in NGC 253 is dominated by large-scale low-velocity shocks.

4. The comparison with Galactic sources also shows a close similarity between the abundances within the nuclear starburst of M82 and those of the Orion Bar, the prototype of a photondominated region. This resemblance fully supports the idea of the chemistry in M82 being dominated by PDRs.

S. M. and R. M. were supported by the Programas de Acciones Integradas between Spain and Germany. J. M. P. has been partially supported by the Spanish Ministerio de Educación y Ciencia under projects ESP 2004-00665, AYA 2002-10113-E and AYA 2003-02785-E. S. G. B. acknowledges financial support from AYA 2003-07584 and ESP 2003-04957. We would like to thank the referee, whose comments helped to significantly improve the paper.

\section{APPENDIX A}

\section{GAUSSIAN FITTING TO BLENDED LINES}

Table 1 includes a label in the third column indicating if and how the respective spectral feature is affected by blending. In the following, the encountered different cases are described:

Blended $(b)$.- - Some observed features are composed of several transitions from different species. If the line is only partially blended, it is still possible to separate the individual components by fitting different Gaussian profiles. For these fits, sometimes it was necessary to fix the velocity and/or line width.

Synthetic $(s)$.- The blending may prevent us from making a reliable multiple Gaussian fitting to an observed feature. In this case, when we have observed several transitions of one of the species contributing to the blended feature, it is possible to use these to estimate the species' contribution to the blended feature by interpolating or extrapolating the linear fit to the rotation diagrams (see $\S$ B1). This is equivalent to using equation (B6) to estimate the expected integrated intensity of a line from the derived column density and rotation temperature. Using the estimated line intensity and the line width and radial velocity of the other observed transitions, we subtract the expected synthetic Gaussian profile from the spectrum. The estimated integrated line intensities using this method have been plotted in the corresponding rotation diagrams with open symbols (Fig. 8). The transitions from other species contributing to the blended feature have been fitted to the residual spectrum after the subtraction of the synthetic profile.

Hyperfine $(h f)$.- - Some species show transitions that consist of a group of unresolved hyperfine components. The relative intensities of these lines are fixed by their spectroscopic parameters, assuming LTE and optically thin line emission. Thus, the fitting has been carried out with a comb of Gaussian profiles at the rest frequencies of the hyperfine components with the same widths and with fixed line intensities relative to the main component, which is taken as a free parameter. The derived parameters shown in Table 1 correspond to the integrated intensity of all the hyperfine components but the frequency, line intensity and velocity refer to the main hyperfine component.

Multitransition $(m)$. - Some features of species such as $\mathrm{CH}_{3} \mathrm{OH}$ and $\mathrm{CH}_{3} \mathrm{CN}$ consist of groups of overlapping transitions, involving different energy levels. In these cases, a single Gaussian profile has been fitted to the observed spectral feature. The total integrated line intensity of all the blended transitions has been used to derive rotation diagrams as described in $\S \mathrm{B} 2$. 

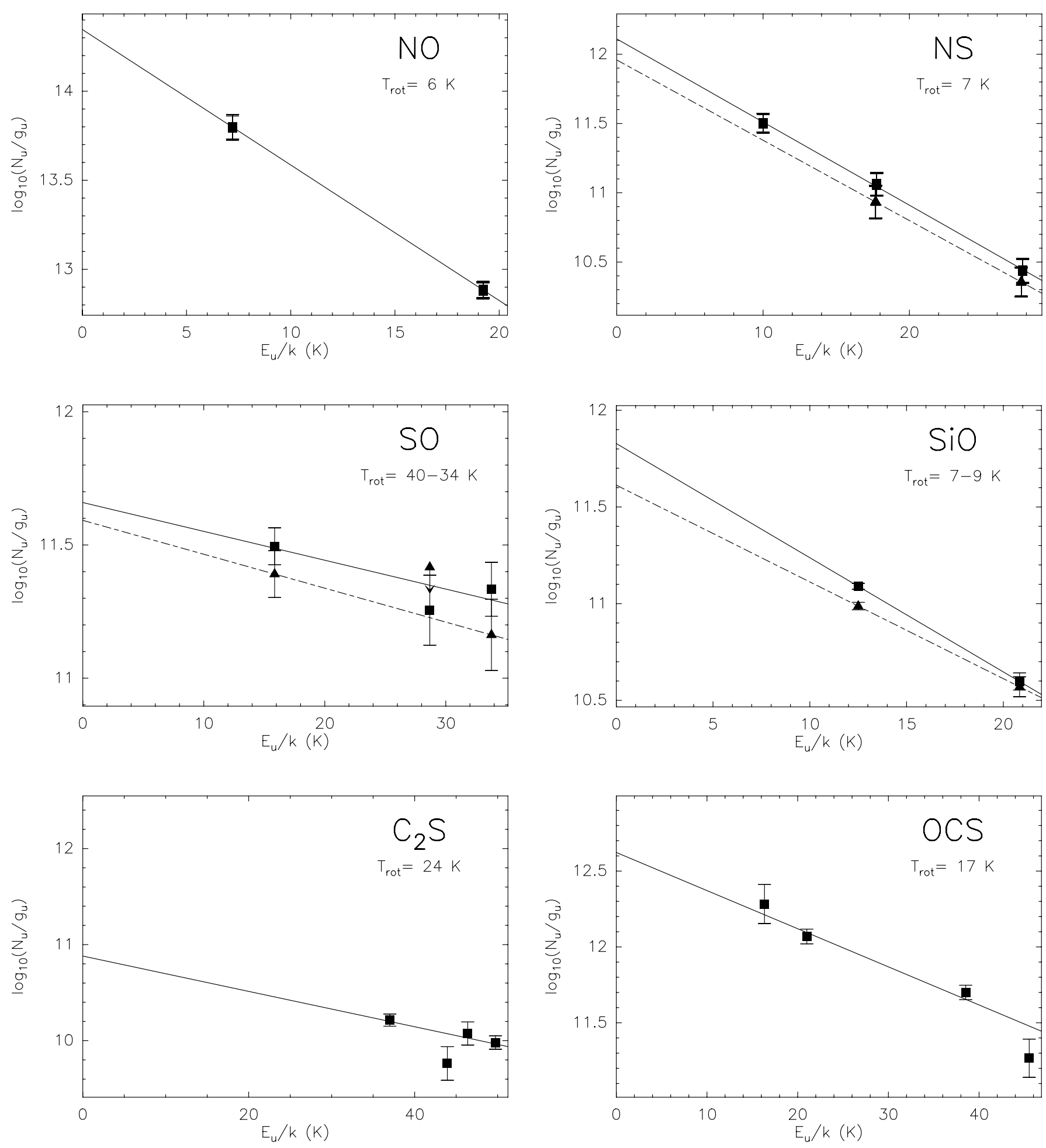

FIG. 8.-Rotation diagrams of detected species. When differentiated, velocity components are represented as filled squares and solid lines $\left(180 \mathrm{~km} \mathrm{~s}{ }^{-1}\right)$ and filled triangles and dashed lines $\left(270 \mathrm{~km} \mathrm{~s}^{-1}\right)$. Unfilled markers represent blended observed transitions whose intensities have been estimated by inter or extrapolation from the diagrams (see Appendix A for details). The NS, NO, and OCS diagrams include additional transitions observed by Martín et al. (2003, 2005). 

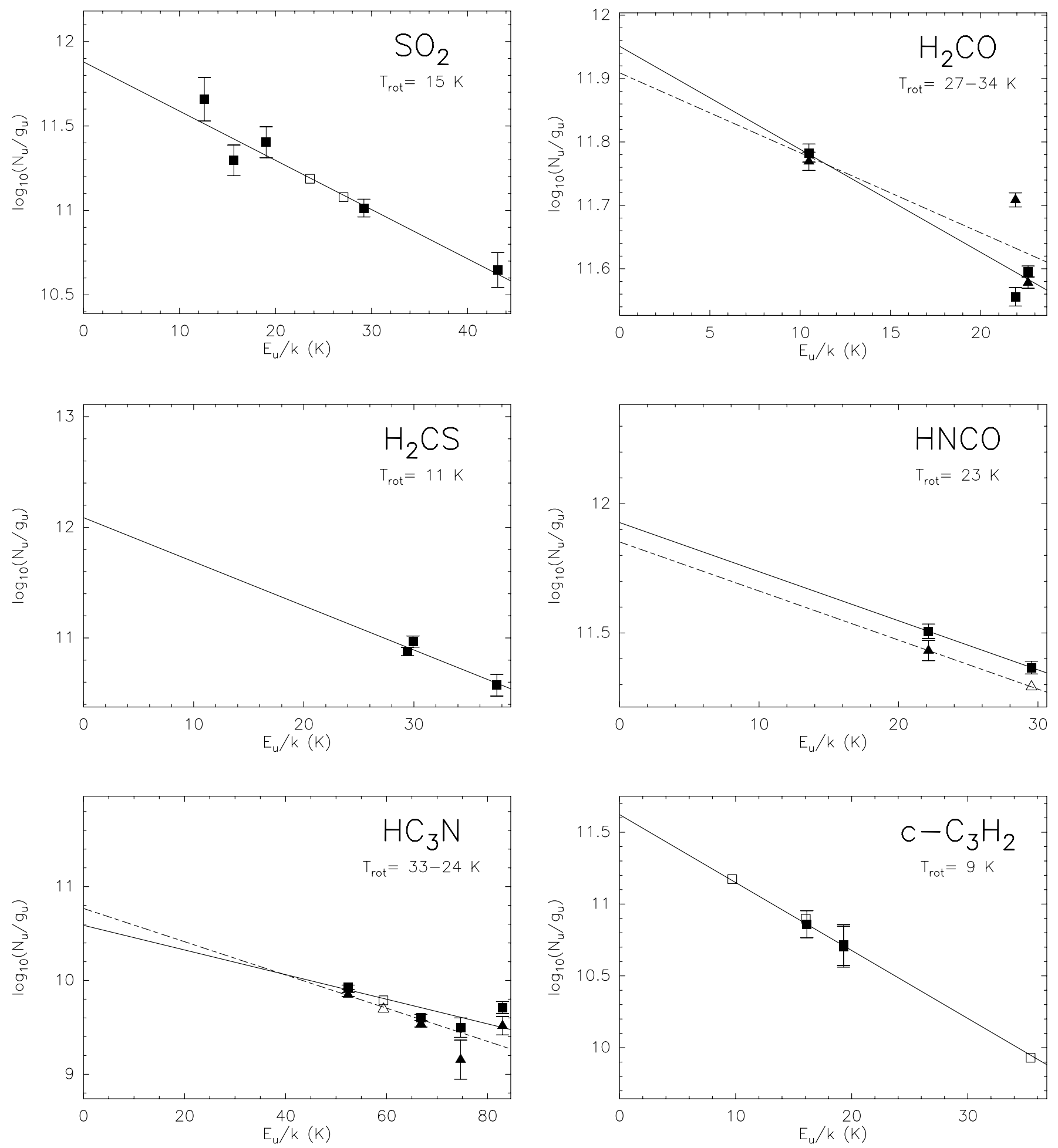

FIg. 8.-Continued 

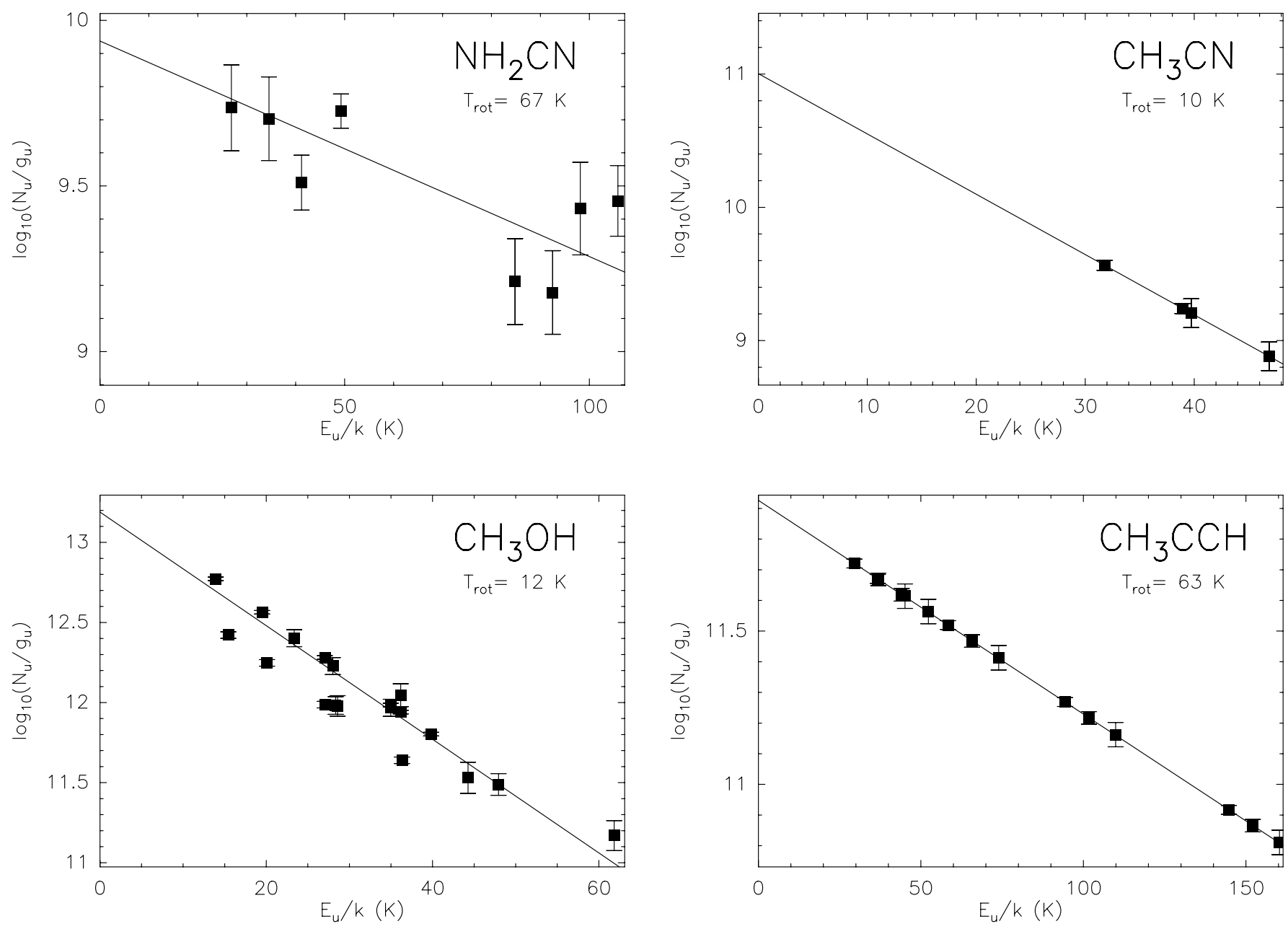

Fig. 8.-Continued

\section{APPENDIX B}

\section{ROTATION DIAGRAMS}

\section{B1. BASICS}

Assuming optically thin emission for the observed molecular transitions, the column density in the upper level can be derived as

$$
N_{u}=\frac{8 \pi k \nu^{2}}{h c^{3} A_{u l}}\left[1-\frac{J_{\nu}\left(T_{\mathrm{BG}}\right)}{J_{\nu}\left(T_{\mathrm{ex}}\right)}\right]^{-1} \int T_{\mathrm{B}} d v,
$$

where $A_{u l}$, the Einstein coefficient for a transition connecting the levels $u$ and $l$, can be written as $A_{u l}=\left(64 \pi^{4} \nu^{3} / 3 h c^{3}\right) S \mu_{u l}^{2} / g_{u}$, and $J_{\nu}(T)=(h \nu / k)[\exp (h \nu / k T)-1]^{-1}$, with $T_{\mathrm{BG}}$ denoting the temperature of the background, $T_{\mathrm{ex}}$ is the excitation temperature of the transition, and $g_{u}$ is the degeneracy of the upper level.

In the Rayleigh-Jeans approximation $(h \nu \ll k T)$, and assuming $T_{\mathrm{ex}} \gg T_{\mathrm{BG}}$, equation (B1) can be reduced to

$$
N_{u}=\frac{8 \pi k \nu^{2}}{h c^{3} A_{u l}} W
$$

where the integrated brightness temperature $\int T_{\mathrm{B}} d v$ is denoted as $W$. If LTE is assumed, the population distribution of all the levels can be represented by a single temperature, the rotation temperature $\left(T_{\text {rot }}\right)$, given by

$$
\frac{N_{u}}{g_{u}}=\frac{N}{Z} e^{-E_{u} / k T_{\text {rot }}},
$$

where $N$ is the total column density, $E_{u}$ is the energy of the upper level, and $Z$ is the partition function calculated as

$$
Z=\sum_{\forall i} g_{i} e^{-E_{i} / k T_{\text {rot }}} .
$$


Using equation (B2) in equation (B3), we obtain

$$
N=\frac{8 \pi k \nu^{2} Z}{h c^{3} A_{u l} g_{u}} W e^{E_{u} / k T_{\mathrm{rot}}}
$$

or the equivalent logarithmic expression,

$$
\log \frac{8 \pi k \nu^{2}}{h c^{3} A_{u l} g_{u}} W=\log \frac{N}{Z}-\frac{\log e E_{u}}{k T_{\text {rot }}},
$$

which constitutes the basic equation for the rotation diagrams (see Blake et al. 1987, Turner 1991, and Goldsmith \& Langer 1999 for a complete description of this method). Plotting $N_{u} / g_{u}$ for each transition against the energy of its upper level in a logarithmic scale, the population distribution can be fitted by a straight line that provides the total column density divided by the partition function as well as $T_{\text {rot }}$.

\section{B2. BLENDED LINES}

Whenever a molecular feature is composed of a number of unresolved blended transitions of a given molecular species, rotation diagrams cannot be directly used to determine the physical properties of the molecular emission. The spectroscopic parameters of the blended individual transitions within an observed feature allow us to estimate the contribution of each transition to the total integrated intensity, assuming LTE and optically thin emission. Thus, using equation (B5) we can express the relative integrated intensity between two transitions with Einstein coefficients $A_{u l}^{\prime}$ and $A_{u l}^{\prime \prime}$, energies of the upper level $E_{u}^{\prime}$ and $E_{u}^{\prime \prime}$, and degeneracies $g_{u}^{\prime}$ and $g_{u}^{\prime \prime}$ as

$$
\frac{W^{\prime}}{W^{\prime \prime}}=\frac{\nu^{\prime \prime 2} A_{u l}^{\prime} g_{u}^{\prime}}{\nu^{\prime 2} A_{u l}^{\prime \prime} g_{u}^{\prime \prime}} e^{\left(E_{u}^{\prime \prime}-E_{u}^{\prime}\right) / k} T_{\mathrm{rot}}^{0}
$$

This allows us to compute the relative intensity of transition for a given $T_{\text {rot }}^{0}$. We will differentiate between the temperature $T_{\text {rot }}^{0}$ we assume for estimating the contribution of each transition to the blended line, and the rotational temperature $T_{\text {rot }}$ derived from the rotation diagram.

If we consider the case of a molecule with hyperfine structure where all the transitions within a feature have the same upper level energy, then we can make the assumptions of $E_{u}^{\prime} \approx E_{u}^{\prime \prime}$ and $\nu^{\prime} \approx \nu^{\prime \prime}$. Therefore, the contribution of each transition will not depend on the assumed $T_{\text {rot }}$ and will only depend on the spectroscopic parameters of each transition in the form

$$
\left(\frac{W^{\prime}}{W^{\prime \prime}}\right)_{\mathrm{HF}}=\frac{A_{u l}^{\prime} g_{u}^{\prime}}{A_{u l}^{\prime \prime} g_{u}^{\prime \prime}}
$$

When the contribution of each hyperfine transition to the total line is computed and plotted on the rotation diagrams, they will all lie in the same point in the rotation diagram (see NO and NS diagrams).

In the general case where $E_{u}^{\prime} \neq E_{u}^{\prime \prime}$, the calculated contribution of each line for a given $T_{\text {rot }}^{0}$ will lie on a straight line when included into the rotation diagram. The line traced by these points will have the slope $\log _{10} e / k T_{\text {rot }}^{0}$.

The LTE approximation assumes that all the transitions will lie in a straight line in the rotation diagram, thus we can modify the assumed $T_{\text {rot }}^{0}$ used to calculate the contributions within the blended lines to obtain the best fit to a straight line to all data in the rotation diagram. At this point we will obtain the situation in which $T_{\text {rot }}^{0} \approx T_{\text {rot }}$.

In this paper we have several examples where this method has been applied, i.e., the $\mathrm{CH}_{3} \mathrm{CN}$ rotation diagram, where only two multiple-transition lines are plotted, the $\mathrm{CH}_{3} \mathrm{CCH}$ diagram, where three blended lines are perfectly aligned to a straight line, and the $\mathrm{CH}_{3} \mathrm{OH}$ diagram, where a mixture of multitransition lines and single lines are plotted altogether (Fig. 8).

We have to take special care when dealing with the symmetric top rotors such as $\mathrm{CH}_{3} \mathrm{CN}$ and $\mathrm{CH}_{3} \mathrm{CCH}$. In this case, the rotation temperature is not sufficient to describe the relative population of the levels given that both $T_{\text {kin }}$ and $T_{\text {rot }}$ are required to evaluate the partition function as described by Turner (1991). Thus, $T_{\text {rot }}$ would characterize the population of $J$ levels within a $K$ ladder, while $T_{\text {kin }}$ would describe the population between $K$ ladders. Our observations do not resolve the transitions of different $K$ ladders. We assume both temperatures to be the same $\left(T_{\text {kin }}=T_{\text {rot }}\right)$ so that the population of all levels is described by a single rotation temperature. Given that $T_{\text {rot }}$ is a lower limit to $T_{\text {kin }}$, with typically $T_{\text {rot }} \ll T_{\text {kin }}$, this assumption may result in an overestimation of the column density derived from rotation diagrams as the contribution to the total integrated intensity of the $K \geq 1$ levels may have been underestimated. On the other hand, the evaluation of the partition function will also be underestimated. Thus, the overall effect in the total column density determination is not expected to be more than a factor of $2-3$, while the derived rotation temperature will only be marginally affected.

\section{APPENDIX C}

\section{NOTES ON INDIVIDUAL MOLECULES}

In the following, we will discuss the fitting procedure of the identified molecular species in detail.

\section{C1. CARBON MONOSULFIDE: CS}

Only the $J=3-2$ transition of carbon monosulfide at $147 \mathrm{GHz}$ is observed in the survey. It appears to be partially contaminated by the $\mathrm{H} 35 \alpha$ recombination line. The estimated Gaussian profile derived from the observed $\mathrm{H} 34 \alpha$ (see $\S \mathrm{C} 26$ ) has been subtracted to account for its contribution. 
Two rare isotopes, ${ }^{13} \mathrm{CS}$ and $\mathrm{C}^{34} \mathrm{~S}$ at 144.6 and $138.8 \mathrm{GHz}$, respectively, are also observed in the spectral scan. Martín et al. (2005) used these observations of CS and its isotopes, as well as additional CS lines, to derive isotope ratios of ${ }^{32} \mathrm{~S} /{ }^{34} \mathrm{~S} \sim 8 \pm 2$ and ${ }^{34} \mathrm{~S} /{ }^{33} \mathrm{~S}>9$. From the observation of additional CS transitions at 3, 1.5, and $1.2 \mathrm{~mm}$, Martín et al. (2005) derive a $T_{\text {rot }} \sim 10 \mathrm{~K}$.

\section{C2. NITRIC OXIDE: NO}

One rotational transition of nitric oxide (150.2 and $150.5 \mathrm{GHZ}$ ) has been identified in our survey. Martín et al. (2003) confirmed this identification by observing additional transitions at $250 \mathrm{GHz}$. These transitions have also been included in the rotation diagram in Figure 8. The topology of the NO energy levels is similar to that of NS. Transitions connecting upper and lower levels are labeled $\Pi^{+}$ and $\Pi^{-}$.

It was only possible to fit the $\Pi^{+}$hyperfine group of the transition at $150.2 \mathrm{GHz}$, given that the $\Pi^{-}$group is blended with the more intense $\mathrm{H}_{2} \mathrm{CO}$ line (see $\S \mathrm{C} 14$ ). As shown in Table 1, the $\Pi^{-}$group of transitions has been estimated by fixing width and radial velocity of each component to the values derived from the fitting to the $\Pi^{+}$transition and the line intensities determined by their relative line strengths.

\section{C3. NITRIC SULFIDE: NS}

One rotational transition of nitric sulfide ( 161.2 and $161.7 \mathrm{GHz}$ ) has been detected in our survey. Martín et al. (2003) confirmed the identification by detecting other transitions at 115 and $207 \mathrm{GHz}$. The rotation diagram in Figure 8 includes these additional transitions.

This molecule presents $\Lambda$-doubling and hyperfine splitting. Because of the $\Lambda$-doubling, each rotational level is divided into two levels with opposite parity. Transitions connecting the upper levels are denoted as $e$ and those connecting the lower levels as $f$ (see Table 1). We are able to resolve the split rotational levels due to $\Lambda$-doubling but the intrinsic line widths prevent us from resolving the hyperfine structure.

\section{C4. SULFUR MONOXIDE: SO}

We detect three transitions of sulfur monoxide $(138.2,159$, and $172.2 \mathrm{GHz})$. The velocity components at 180 and $275 \mathrm{~km} \mathrm{~s}^{-1}$ are clearly identified. As seen in the rotation diagrams (Fig. 8), the $275 \mathrm{~km} \mathrm{~s}^{-1}$ component of the $3_{4}-2_{3}$ transition shows a much higher intensity than expected if we assume both components to have the same rotation temperature. This is likely due to the contamination of this component by the $8_{1,8}-7_{1,7}$ transition of $\mathrm{NH}_{2} \mathrm{CN}$. The uncertainty in the contribution of this line due to the noise strongly affects the fitting of the high-velocity component of this SO transition.

One of the isotopic substitutions of sulfur monoxide, ${ }^{34} \mathrm{SO}(135.8 \mathrm{GHz})$ is detected blended with a $\mathrm{SO}_{2}$ transition. This faint feature has been fitted with a double Gaussian, with the velocity and width of each component fixed to the values derived from the same transition, $4_{3}-3_{2}$, of the main isotope. We calculate a ratio ${ }^{32} \mathrm{SO} /{ }^{34} \mathrm{SO}=5.1 \pm 1.2$, using both velocity components of the $4_{3}-3_{2}$ transition of both isotopes. This ratio is consistent within the errors with ${ }^{32} \mathrm{~S} /{ }^{34} \mathrm{~S}=8 \pm 2$ as derived by Martín et al. (2005) from CS data.

\section{C5. SILICON MONOXIDE: SIO}

Two transitions of silicon monoxide in the ground vibrational state are observed at 130.3 and $173.7 \mathrm{GHz}(J=3-2$ and $J=4-3$, respectively). It is possible to identify two velocity components from the $J=3-2$ transition at 180 and $260 \mathrm{~km} \mathrm{~s}^{-1}$. Velocity and width of the fainter and more noisy $J=4-3$ transition were fixed to the values obtained from the fit to the $J=3-2$ line. The rotation diagram has been derived for each velocity component (Fig. 8, solid and dashed lines).

\section{C6. HYDROGEN SULFIDE: $\mathrm{H}_{2} \mathrm{~S}$}

Only one line of hydrogen sulfide at $168.8 \mathrm{GHz}$, with velocity components at 180 and $275 \mathrm{~km} \mathrm{~s}^{-1}$, is detected in the $2 \mathrm{~mm}$ survey (see also Martín et al. 2005). Physical parameters in Table 2 have been derived by assuming a rotation temperature of $12 \mathrm{~K}$ similar to that derived for many other species (see Table 2).

\section{C7. HYDROCYANIC ACID: HCN}

The $J=2-1$ transition of $\mathrm{HCN}$ at $177.2 \mathrm{GHz}$ lies outside the covered frequency range, but the $2-1$ line of its $\mathrm{H}^{13} \mathrm{CN}$ isotope at $172.6 \mathrm{GHz}$ was detected. A fit to this transition allows us to estimate the abundance of $\mathrm{HCN}$ if we assume a rotation temperature of $12 \mathrm{~K}$ and the ${ }^{12} \mathrm{C} /{ }^{13} \mathrm{C}$ ratio of 40 derived by Henkel et al. (1993).

\section{C8. OXOMETHYLIUM: $\mathrm{HCO}^{+}$}

The only $2 \mathrm{~mm}$ transition of the main species of oxomethylium, with energy levels low enough to be observable in the ISM of NGC 253 , at a frequency of $178.3 \mathrm{GHz}$, lies a few $\mathrm{GHz}$ above the observed frequency range. Nevertheless, one of its isotopes, $\mathrm{H}^{13} \mathrm{CO}^{+}$, has been detected in the $J=2-1$ transition at $173.5 \mathrm{GHz}$, near the upper end of the spectral scan. Two velocity components can be fitted to the observed profile. The $\mathrm{HCO}^{+}$column density shown in Table 2 has been calculated from the integrated intensity of the measured $\mathrm{H}^{13} \mathrm{CO}^{+}$line with an excitation temperature of $12 \mathrm{~K}$ and an isotopic ratio of ${ }^{12} \mathrm{C} /{ }^{13} \mathrm{C} \sim 40$ (Henkel et al. 1993).

The $J=2-1$ transition of $\mathrm{HC}^{18} \mathrm{O}^{+}$is also identified at $170.3 \mathrm{GHz}$. The low-velocity component shows a higher intensity than expected from the observed $\mathrm{H}^{13} \mathrm{CO}^{+}$profile as well as a slightly higher velocity. If we compare both profiles, we derive a $\mathrm{H}^{13} \mathrm{CO}^{+} /$ $\mathrm{HC}^{18} \mathrm{O}^{+}$ratio of $1.7 \pm 1.1$ and $2.9 \pm 1.2$ for the low- and high-velocity components, respectively. The value derived for the high-velocity 
component agrees within the errors with the expected value of $\sim 3.7$ if we assume the isotopic ratios ${ }^{12} \mathrm{C} /{ }^{13} \mathrm{C}=40$ (Henkel et al. 1993) and ${ }^{16} \mathrm{O} /{ }^{18} \mathrm{O}=150$ (Harrison et al. 1999).

\section{C9. HYDROISOCYANIC ACID: HNC}

Similar to $\mathrm{HCN}$, the $J=2-1$ transition of $\mathrm{HNC}$ at $181.3 \mathrm{GHz}$ lies outside the frequency range of the survey. We identify a feature at 174.1 GHz as the $J=2-1$ transition of $\mathrm{HN}^{13} \mathrm{C}$. The detection is uncertain and thus tentative as the line is strongly blended with the also tentatively identified $3_{1,2}-2_{1,1}$ transition of $c-\mathrm{C}_{3} \mathrm{H}$ (see $\S \mathrm{C} 18$ ).

\section{C10. ETHYNYL: $\mathrm{C}_{2} \mathrm{H}$}

The $J=2-1$ group of hyperfine transitions of $\mathrm{C}_{2} \mathrm{H}$, observed at $174.7 \mathrm{GHz}$ near the upper frequency cutoff of the survey, is the second brightest feature observed in the $2 \mathrm{~mm}$ window after CS. Even though the hyperfine structure is unresolved, it is still possible to differentiate the two velocity components at 160 and $280 \mathrm{~km} \mathrm{~s}^{-1}$.

\section{C11. THIOXOETHENYLIDENE: $\mathrm{C}_{2} \mathrm{~S}$}

The survey shows four faint lines of thioxoethenylidene (131.6, 140.2, 142.5, and 144.2 GHz). The low intensity of the observed transitions makes the fitting uncertain (see Table 1). Only the $11_{10}-10_{9}$ and $10_{11}-9_{10}$ transitions at 131.6 and $140.2 \mathrm{GHz}$ are reliably detected, while the transitions at 142.5 and $144.2 \mathrm{GHz}$ are tentative.

\section{C12. CARBON OXIDE SULFIDE: OCS}

Two transitions of carbon oxide sulfide ( 133.8 and $145.9 \mathrm{GHz}$ ) are detected in the survey. In the rotation diagram in Figure 8 these observations are complemented by the $3 \mathrm{~mm}$ OCS transitions presented by Martín et al. (2005). The $J=13-12$ transition of OCS at $158.1 \mathrm{GHz}$ might have been detected close to a $\mathrm{SO}_{2}$ line, but the signal-to-noise ratio is not high enough for a reliable Gaussian fit.

\section{C13. SULFUR DIOXIDE: $\mathrm{SO}_{2}$}

Five transitions of sulfur dioxide (134.0, 135.7, 140.3, 146.6 and $151.4 \mathrm{GHz})$ have been identified. The upper energy levels of the transitions range from 12 to $43 \mathrm{~K}$ providing a good estimate of the rotation temperature.

The $5_{1,5}-4_{0,4}$ transition at $135.7 \mathrm{GHz}$ is blended with a ${ }^{34} \mathrm{SO}$ line (see $\left.\S \mathrm{C} 4\right)$. Velocity and width of the line were fixed to perform a two Gaussian fit for the $\mathrm{SO}_{2}$ and ${ }^{34} \mathrm{SO}$ transitions.

The $2_{2,0}-2_{1,1}$ line at $151.4 \mathrm{GHz}$ is contaminated by emission of a much fainter $c-\mathrm{C}_{3} \mathrm{H}_{2}$ line, which, according to its estimated intensity (see $\S \mathrm{C} 21$ ), would account for $\sim 15 \%$ of the observed feature. The contribution of the $c-\mathrm{C}_{3} \mathrm{H}_{2}$ strongly depends on the assumed $T_{\text {rot }}$. If we consider the uncertainty in the $T_{\text {rot }}$ derived from $c-\mathrm{C}_{3} \mathrm{H}_{2}$ and estimate the contribution of this transition to the observed $\mathrm{SO}_{2}$ line, the effect on the derived $\mathrm{SO}_{2}$ parameters would be of $\pm 1 \mathrm{~K}$ in $T_{\text {rot }}$ and $\pm 5 \%$ in the column density.

The $5_{2,4}-5_{1,5}$ and $7,7-6_{0,6}$ transitions are blended with a $\mathrm{CH}_{3} \mathrm{OH}$ group of lines at $165.1 \mathrm{GHz}$. We have estimated their contribution to the observed feature by assuming a $T_{\text {rot }}=15 \mathrm{~K}$ as derived from the rotation diagram. For the derived excitation conditions of $\mathrm{SO}_{2}$, its $3_{2,2}-3_{1,3}$ line at $158.1 \mathrm{GHz}$ is expected to have an intensity of $\sim 5 \mathrm{mK}$, which is below the noise level at this frequency.

\section{C14. FORMALDEHYDE: $\mathrm{H}_{2} \mathrm{CO}$}

Two ortho (140.8 and $150.5 \mathrm{GHz})$ and one para $(145.6 \mathrm{GHz}) J=2-1$ transitions of formaldehyde are detected. In each of the profiles, the two velocity components at 180 and $285 \mathrm{~km} \mathrm{~s}^{-1}$ are clearly separated.

The $2_{0,2}-1_{0,1}$ transition at $145.6 \mathrm{GHz}$ and the $2_{1,1}-1_{1,0}$ at $150.4 \mathrm{GHz}$ are blended with an $\mathrm{HC}_{3} \mathrm{~N}$ and a $c-\mathrm{C}_{3} \mathrm{H}_{2}$ line, respectively. Before performing the Gaussian fits to the $\mathrm{H}_{2} \mathrm{CO}$ features, the estimated contributions from $\mathrm{HC}_{3} \mathrm{~N}$ (see $\left.\S \mathrm{C} 19\right)$ and $c-\mathrm{C}_{3} \mathrm{H}_{2}(\operatorname{see} \S \mathrm{C} 21)$ were subtracted from the spectra.

Rotation diagrams are plotted for each velocity component (Fig. 8) where rotation temperatures of 27 and $34 \mathrm{~K}$ are derived for the 180 and $285 \mathrm{~km} \mathrm{~s}^{-1}$ components, respectively. The $285 \mathrm{~km} \mathrm{~s}^{-1}$ component of the $2_{1,2}-1_{1,1}$ transition appears to have a significantly higher intensity than expected from the rotation diagram. The large intensity of the $2_{1,2}-1_{1,1}$ line of $\mathrm{H}_{2} \mathrm{CO}$ could be due to the contamination by an unidentified line. If this measurement is not taken into account, the resulting temperature derived for the $285 \mathrm{~km} \mathrm{~s}^{-1}$ component would be $28 \pm 2 \mathrm{~K}$, while the estimated column density would be lower by $\sim 30 \%$, closer to the parameters derived from the $180 \mathrm{~km} \mathrm{~s}^{-1}$ component.

\section{C15. THIOFORMALDEHYDE: $\mathrm{H}_{2} \mathrm{CS}$}

Three transitions, all belonging to ortho-thioformaldehyde $(135.3,139.5$, and $169.1 \mathrm{GHz})$, are tentatively detected. The $4_{1,4}-3_{1,3}$ transition is blended with the $\mathrm{H} 36 \alpha$ recombination line. A Gaussian profile similar to that of the observed $\mathrm{H} 34 \alpha$ line at $160 \mathrm{GHz}$ (see $\S \mathrm{C} 26)$ has been subtracted. We assume that the residual emission is due to $\mathrm{H}_{2} \mathrm{CS}$. We also find the $4_{1,3}-3_{1,2}$ line blended with the CS $J=3-2$ emission from the image band. With the known image sideband rejection at this frequency (Fig. 3), we can estimate the contribution of the CS line measured at $146.9 \mathrm{GHz}(\S \mathrm{C} 1)$. The resulting feature after the subtraction of the CS line is fitted by a Gaussian profile. There is a part of this feature that is not properly fitted by emission from CS and $\mathrm{H}_{2} \mathrm{CS}$. This may be caused by a slight difference in the observing position between this spectrum and that containing CS in the signal band, which would cause an 
appreciable change in the shape of the subtracted line. Contamination by a third species is unlikely. The $5_{1,5}-4_{1,4}$ line at $169.1 \mathrm{GHz}$ is not blended, but the relatively high noise level at this frequency and the presence of nearby lines makes the fit uncertain.

A rotation temperature of $11 \mathrm{~K}$ is determined from the rotation diagram (Fig. 8) and is consistent with those derived from other molecules. With this rotation temperature, the $4_{0,4}-3_{0,3}$ transition of para- $\mathrm{H}_{2} \mathrm{CS}$ at $137.3 \mathrm{GHz}$ is expected to have an intensity of $\sim 6 \mathrm{mK}$. The nondetection of this line could be explained by an excitation temperature of $>20 \mathrm{~K}$.

\section{C16. ISOCYANIC ACID: HNCO}

The two brightest lines of isocyanic acid in the covered frequency band are clearly detected (131.9 and $153.9 \mathrm{GHz})$. The $280 \mathrm{~km} \mathrm{~s}^{-1}$ component of the $7_{0,7}-6_{0,6}$ transitions at $153.9 \mathrm{GHz}$ is blended with a group of $\mathrm{CH}_{3} \mathrm{CCH}$ lines. Thus, the contribution of this component has been calculated by assuming the 180 and $280 \mathrm{~km} \mathrm{~s}^{-1}$ velocity components have the same excitation temperature. This assumption is supported by the good fit of the $\mathrm{CH}_{3} \mathrm{CCH}$ line to the residual feature (see $\S \mathrm{C} 25$ ). The line observed at $154.4 \mathrm{GHz}$ is tentatively identified as the $280 \mathrm{~km} \mathrm{~s}^{-1}$ velocity component of the $\mathrm{HNCO} 7_{1.6}-6_{1,5}$ transition. The $7_{1,7}-6_{1,6}$ transition at $153.3 \mathrm{GHz}$, which should have a similar intensity, is not detected. Due to the uncertainty of this identification, this line has not been used in the analysis of the HNCO excitation conditions. Including this transition in the rotation diagram (Fig. 8) would result in a derived $T_{\text {rot }} \sim 29 \mathrm{~K}$, slightly higher than that derived for the $180 \mathrm{~km} \mathrm{~s}^{-1}$ velocity component, and a column density $\sim 16 \%$ lower than that in Table 2 .

\section{C17. PORTMANTEAU CARBON DIOXIDE: $\mathrm{HOCO}^{+}$}

We tentatively identified two transition $(149.6$ and $171.0 \mathrm{GHz}$ ) of portmanteau carbon dioxide, a quasilinear molecular ion and nearly symmetric top. These are the brightest observable transitions in the $2 \mathrm{~mm}$ range. Only the high-velocity component of both lines are detected. The large intensity observed in the $8_{1,8^{-}} 7_{1,7}$ transitions, expected to be similar or lower than the $7_{0,7}-6_{0,6}$, is mainly due to the uncertainties of the baseline substraction. A rotational temperature of $12 \mathrm{~K}$ have been assumed to estimate an average column density with both detected transitions. Further observations of other $\mathrm{HOCO}^{+}$transitions are needed to confirm the detection and constrain the excitation temperature of this molecule.

\section{C18. CYCLOPROPENYLIDYNE: $c-\mathrm{C}_{3} \mathrm{H}$}

The $3_{1,2}-2_{1,1}$ group of transitions at $174 \mathrm{GHz}$ is the only feature of $c-\mathrm{C}_{3} \mathrm{H}$ identified in the survey. Contamination by the feature by the $J=2-1$ transition of $\mathrm{HN}^{13} \mathrm{C}$ makes the fitting uncertain. Thus, the velocity component were fixed to $V_{\mathrm{LSR}}=180$ and $280 \mathrm{~km} \mathrm{~s}^{-1}$ as derived for most species and the width of the lower velocity component to $\Delta v_{1 / 2}=41 \mathrm{~km} \mathrm{~s}^{-1}$ similar to that from the fitting to the high-velocity one. The $4_{1,4}-3_{1,3}$ transitions at $172.6 \mathrm{GHz}$ appear to be blended with a $\mathrm{H}^{13} \mathrm{CN}$ line. At $132.9 \mathrm{GHz}$, the $3_{1,3}-2_{1,2}$ group of transitions, which is expected to be similar in intensity to those at $174 \mathrm{GHz}$, is likely present blended to a transition of $\mathrm{CH}_{2} \mathrm{NH}^{2}$ and an also likely transition of $\mathrm{CH}_{3} \mathrm{OH}$, but the noise level at these frequencies do not allow us to separate these lines. Given the considered source size of $20^{\prime \prime}$ the $133 \mathrm{GHz}$ line would be $20 \%$ fainter than that at $174 \mathrm{GHz}$, so beam dilution cannot account for the nondetection of these lines. Therefore, its detection, similar to the $\mathrm{HN}^{13} \mathrm{C}$ line to which it is blended (§ $\left.\mathrm{C} 9\right)$ is highly tentative.

\section{C19. CYANOACETYLENE: $\mathrm{HC}_{3} \mathrm{~N}$}

Four out of the five transitions of cyanoacetylene detected in the observed frequency range (136.5, 145.6, 154.7, 163.8, and 172.8 GHz) appear not to be blended with any other molecular line. Two velocity components have been fitted to each line. The $J=16-15$ transition at $145.6 \mathrm{GHz}$ is blended with an $\mathrm{H}_{2} \mathrm{CO}$ line. Since we have observed more $\mathrm{HC}_{3} \mathrm{~N}$ than $\mathrm{H}_{2} \mathrm{CO}$ lines to accurately determine the physical parameters, we have estimated the relative contribution of each of the velocity components of $\mathrm{HC}_{3} \mathrm{~N}$. For the $\mathrm{HC}_{3} \mathrm{~N} J=18-17$ line, where the velocity components are not clearly differentiated, the velocity of each component has been fixed. The $J=19-18$ transition seems to lie above the $T_{\text {rot }} \sim 20 \mathrm{~K}$ derived from the other observed transitions. This may indicate that the lower energy transitions would not be optically thin as blending with any other molecular line seems unlikely. Multiline observations by Mauersberger et al. (1990; toward a position $9^{\prime \prime}$ north of our position) also noticed an excess of the higher transition lines of $\mathrm{HC}_{3} \mathrm{~N}$. Their comparison with model computations show that two molecular components are required to explain the observed line intensities, one with $n\left(\mathrm{H}_{2}\right) \sim 10^{4} \mathrm{~cm}^{-3}$ and $T_{\text {kin }} \sim 60 \mathrm{~K}$ and a second with $n\left(\mathrm{H}_{2}\right) \sim 5 \times 10^{5} \mathrm{~cm}^{-3}$ and $T_{\text {kin }} \sim 150 \mathrm{~K}$. The line intensity of the $J=17-16$ transition measured in this work is about half that measured by Mauersberger et al. (1990), which indicates the strong effect of a pointing offset in the total integrated intensity. However, as far as the physical parameters are concerned, the use of the $J=19-18$ line, although causing a change in the determined rotation temperature of $\sim 50 \%$, affects the total column density by less than a factor of 2 .

\section{C20. METHANIMINE: $\mathrm{CH}_{2} \mathrm{NH}$}

One transition of methanimine is clearly identified in the line scan. The observed profile of the $2_{1,1}-1_{1,0}$ transition at $133 \mathrm{GHz}$ is heavily affected by nonlinear baselines, and the fit shown in Table 1 is uncertain. The $1_{1,0}-1_{0,1}$ transition at $167 \mathrm{GHz}$, expected to have an intensity twice as large as that at $133 \mathrm{GHz}$, is not reliably detected due to the lack of sensitivity at this frequency. Although the detection seems to be clear, further observations of transitions in other frequency bands are needed to confirm this detection.

\section{C21. CYCLOPROPENYLIDENE: $c-\mathrm{C}_{3} \mathrm{H}_{2}$}

Three transitions of cyclopropenylidene $\left(155.5\right.$ and $150.8 \mathrm{GHz}$ ) are clearly detected. However, only one transition, the $3_{2,2}-2_{1,1}$ line at $155.5 \mathrm{GHz}$, appears not to be blended with any other line. 


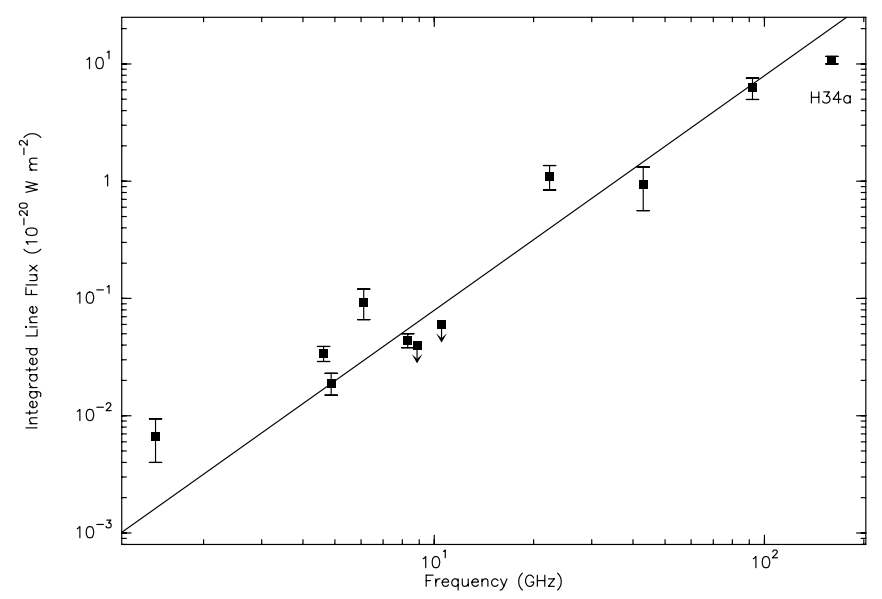

FIg. 9.- Recombination line fluxes of measured $\mathrm{H} \alpha$ lines in the range from 1 to $160 \mathrm{GHz}$ as a function of frequency. A best-fit to the line fluxes in the form $S_{L} \propto \nu^{2}$ is shown as fitted by Puxley et al. (1997).

The $4_{0,4}-3_{1,3}$ and $4_{1,4}-3_{0,3}$ lines, both at $150.8 \mathrm{GHz}$, appear to be blended. A double Gaussian profile was fitted by assuming equal width and velocity and a line intensity ratio determined by their spectroscopical parameters, assuming optically thin emission.

The intensities of the other blended transitions (145.1 and $150.4 \mathrm{GHz}$ ) were estimated for a rotation temperature of $9 \mathrm{~K}$ as derived from the rotation diagram (Fig. 8). Given the small dynamic range in energies of the unblended transitions used in the rotation diagram, the estimated $T_{\text {rot }}$ is affected by a large uncertainty (see Table 2) and, therefore, the estimates for the blended transitions are also quite uncertain. The $5_{1,4}-5_{0,5}$ transition at $151.3 \mathrm{GHz}$ with an estimated intensity of $\sim 1 \mathrm{mK}$, well below the noise level of the spectra, has been calculated with the aim of estimating its contribution to the faint $\mathrm{SO}_{2}$ transition at that frequency $(\S \mathrm{C} 13)$.

\section{C22. CYANAMIDE: $\mathrm{NH}_{2} \mathrm{CN}$}

Only one line of cyanamide is clearly detected in the survey, the $8_{1,7}-7_{1,6}$ transition at $161.0 \mathrm{GHz}$, which appears to be partially blended with image sideband emission from $\mathrm{H}_{2} \mathrm{~S}$. The emission of $\mathrm{H}_{2} \mathrm{~S}$ in the signal band has been corrected by the image sideband rejection and subtracted from the spectrum before fitting the $\mathrm{NH}_{2} \mathrm{CN}$ line. The other observed lines are close to the noise level, and therefore all fitted parameters are strongly affected by the baseline. The $8_{1,8}-7_{1,7}$ transition at $158.8 \mathrm{GHz}$, seems to be blended with a $\mathrm{SO}\left(\S_{\mathrm{C}} 4\right)$ but the noise level does not permit a fit. The high rotation temperature of $63 \mathrm{~K}$ derived from the rotation diagram (Fig. 8) depends mainly on the fainter and therefore less reliably fitted transitions. A much lower rotation temperature would lower the column density by a factor of about 2 .

\section{C23. METHYL CYANIDE: $\mathrm{CH}_{3} \mathrm{CN}$}

Two spectral features are identified as methyl cyanide transitions (147.2 and $165.6 \mathrm{GHz})$. Each of the $J-J^{\prime}$ transitions of this symmetric top consist of a number of overlapped $K$ components $(K=0 \ldots J-1)$. Single Gaussian profiles were fitted to the observed lines. The contribution of the $K=0$ and 1 ladders, for the low rotation temperatures derived from other high dipole moment molecules, account for $\sim 98 \%$ of the line profile. Therefore, the $K>1$ contribution to the total line intensity has not been taken into account. We derive a $T_{\text {rot }}=10 \mathrm{~K}$ from the rotation diagram in Fig. 8, which has been obtained as explained in $\S$ B2.

\section{C24. METHANOL: $\mathrm{CH}_{3} \mathrm{OH}$}

A total of nine transitions or groups of transitions of methanol is detected in the line survey at 143.9, 145.1, 146.4, 157, 165, and $170 \mathrm{GHz}$. The transitions at $145.1 \mathrm{GHz}$ are blended with the $\mathrm{C}_{3} \mathrm{H}_{2} 3_{1,2}-2_{2,1}$ line, whose contribution has been estimated (see $\S \mathrm{C} 21$ ) and subtracted from the observed feature. In the same way, the lines at $165.0 \mathrm{GHz}$ are slightly blended with $\mathrm{SO}_{2}$ transitions. The emission of these $\mathrm{SO}_{2}$ lines, subtracted from the spectra (see $\S \mathrm{C} 13$ ), mainly affects the fit of the $4_{1,3}-4_{0,4}$ transition. The $6_{-1}-5_{0}$ transition of $\mathrm{CH}_{3} \mathrm{OH}$ expected to be detected at $132.8 \mathrm{GHz}$ is heavily affected by baseline instabilities. The rotation diagram derived from the observed transitions has been plotted using both the single- and multitransition lines as described in $\S \mathrm{B} 2$. The derived rotation temperature is $12 \mathrm{~K}$.

\section{C25. METHYL ACETYLENE: $\mathrm{CH}_{3} \mathrm{CCH}$}

Three methyl acetylene features are observed $(136.7,153.8$, and $170.9 \mathrm{GHz})$. As in the case of $\mathrm{CH}_{3} \mathrm{CN}$, each of these corresponds to a $\Delta J=1$ transition consisting of a number of unresolved $K$ components (with $K=0 \ldots J-1$ ). Single Gaussian profiles have been fitted to the observed profiles.

The best fit to the rotation diagram as described in $\S \mathrm{B} 2$ results in a rotation temperature of $\sim 62 \mathrm{~K}$. Given the high rotation temperature derived, only contributions of transitions with $K=0 \ldots 4$, which have upper level energies $<160 \mathrm{~K}$, have been taken into account in the fit. For a temperature of $60 \mathrm{~K}$, transitions with $K=4$ contribute $\sim 5 \%$ to the observed profiles, while higher $K$ lines represent less than $1 \%$ of the integrated intensities. Neglecting the contribution of these higher energy transitions (i.e., assuming the 
whole component is dominated by the $K=0$ transitions) would result in an overestimation of the column densities by up to a factor of 4 .

\section{C26. HYDROGEN RECOMBINATION LINES}

Four $\mathrm{H} \alpha$ recombination lines are present within the frequency range covered by our survey. Only one of them, $\mathrm{H} 34 \alpha$, is not blended and can be fitted. The $\mathrm{H} 33 \alpha$ line lies close to the $2-1$ line of $\mathrm{C}_{2} \mathrm{H}$ at the upper end of the frequency range where the rms is similar to the expected intensity of the line. The H35 $\alpha$ and $\mathrm{H} 36 \alpha$ lines are blended with CS $3-2$ and $\mathrm{H}_{2} \mathrm{CS} 4_{1,4}-3_{1,3}$ lines, respectively. If the relation of the line flux $S_{\mathrm{L}} \propto \nu_{\mathrm{L}}^{2}$ applies at these frequencies (Puxley et al. 1997), the lines should have flux densities $20 \%$ and $30 \%$ below that measured for the $\mathrm{H} 34 \alpha$ line. We have considered them to have a similar line profile to that of the measured $\mathrm{H} 34 \alpha$ line given the uncertainty in the fitted intensity.

To convert the measured $\mathrm{H} 34 \alpha$ line temperature into flux densities, we use the conversion factor for the $30 \mathrm{~m}$ telescope $S / T_{\mathrm{MB}}=$ $4.95 \mathrm{Jy} \mathrm{K}{ }^{-1}$. Following Puxley et al. (1997), a source to beam coupling factor $\left(\theta_{b}^{2}+\theta_{s}^{2}\right) / \theta_{b}^{2}$ has been applied for a source size $\theta_{s}=7^{\prime \prime}$. We calculate an integrated line flux of $(1.08 \pm 0.08) \times 10^{-19} \mathrm{~W} \mathrm{~m}^{-2}$. As seen in Figure 9 , this value closely follows the best fit to the relation between the integrated line flux versus frequency derived by Puxley et al. (1997). In this figure the flux of the H34 $\alpha$ line is plotted together with the recombination lines observed in millimeter and centimeter wavelengths (Puxley et al. 1997 and references therein).

\section{REFERENCES}

Blake, G. A., Sutton, E. C., Masson, C. R., \& Phillips, T. G. 1987, ApJ, 315, 621

Bradford, C. M., et al. 1999, in The Universe as Seen by ISO, ed. P. Cox \& M. F. Kessler (ESA-SP 427; Noordwijk: ESA), 861

Burles, S. 2002, Planet. Space Sci., 50, 1245

Canzian, B., Mundy, L. G., \& Scoville, N. Z. 1988, ApJ, 333, 157

Cernicharo, J., Guélin, M., \& Kahane, C. 2000, A\&AS, 142, 181

Charnley, S. B., Kress, M. E., Tielens, A. G. G. M., \& Millar, T. J. 1995, ApJ, 448, 232

Chin, Y.-N., Henkel, C., Langer, N., \& Mauersberger, R. 1999, ApJ, 512, L143

Chin, Y.-N., Henkel, C., Millar, T. J., Whiteoak, J. B., \& Mauersberger, R. 1996, A\&A, 312, L33

Churchwell, E., Witzel, A., Huchtmeier, W., Pauliny-Toth, I. I. K., Roland, J., \& Sieber, W. 1977, A\&A, 54, 969

Cummins, S. E., Linke, R. A., \& Thaddeus, P. 1986, ApJS, 60, 819

Das, M., Anantharamaiah, K. R., \& Yun, M. S. 2001, ApJ, 549, 896

de Vaucouleurs, G., et al. 1991, Third Reference Catalogue of Bright Galaxies (Berlin: Springer)

de Vicente, P., Martín-Pintado, J., \& Wilson, T. L. 1997, A\&A, 320, 957

Downes, D. Radford, S. J. E., Guilloteau, S., Guelin, M., Greve, A., \& Morris, D. 1992, A\&A, 262, 424

Engelbracht, C. W., Rieke, M. J., \& Rieke, G. H. 1998, ApJ, 505, 639

Flower, D. R., Pineau des Forêts, G., \& Walmsley, C. M. 1995, A\&A, 294, 815

Forbes, D. A., \& Depoy, D. L. 1992, A\&A, 259, 97

Fossé, D., Cesarsky, D., Gerin, M., Lequeux, J., \& Tiné, S. 2000, in ISO beyond the Peaks: The 2nd ISO Workshop on Analytical Spectroscopy, ed. A. Salama, M. F. Kessler, K. Leech, \& B. Schulz (ESA-SP 456; Noordwijk: ESA), 91

Frayer, D. T., Seaquist, E. R., \& Frail, D. A. 1998, AJ, 115, 559

Friedel, D. N., Snyder, L. E., Turner, B. E., \& Remijan, A. 2004, ApJ, 600, 234

Fuente, A., Black, J. H., Martín-Pintado, J., Rodríguez-Franco, A., GarcíaBurillo, S., Planesas, P., \& Lindholm, J. 2000, ApJ, 545, L113

Fuente, A., Martín-Pintado, J., Cernicharo, J., \& Bachiller, R. 1993, A\&A, 276, 473

Fuente, A., Rodríguez-Franco, A., García-Burillo, S., Martín-Pintado, J., \& Black, J. H. 2003, A\&A, 406, 899

Fuente, A., Rodríguez-Franco, A., \& Martín-Pintado, J. 1996, A\&A, 312, 599

García-Burillo, S., Martín-Pintado, J., Fuente, A., \& Neri, R. 2000, A\&A, 355, 499

García-Burillo, S., Martín-Pintado, J., Fuente, A., \& Usero, A. 2002, ApJ, 575, L55

Gardner, F. F., \& Whiteoak, J. B. 1974, Nature, 247, 526

Goicoechea, J. R., Martín-Pintado, J, \& Cernicharo, J 2005, ApJ, 619, 291

Goldsmith, P. F., \& Langer, W. D. 1999, ApJ, 517, 209

Harrison, A., Henkel, C., \& Russell, A. 1999, MNRAS, 303, 157

Hasegawa, T., Sato, F., Whiteoak, J. B., \& Miyawaki, R. 1994, ApJ, 429, L77

Heikkilä, A., Johansson, L. E. B., \& Olofsson, H. 1999, A\&A, 344, 817

Henkel, C., \& Bally, J. 1985, A\&A, 150, L25

Henkel, C., Chin, Y.-N., Mauersberger, R., \& Whiteoak, J. B. 1998, A\&A, 329, 443

Henkel, C., Jacq, T., Mauersberger, R., Menten, K. M., \& Steppe, H. 1987, A\&A, 188, L1

Henkel, C., Mauersberger, R., Peck, A. B., Falcke, H., \& Hagiwara, Y. 2000, A\&A, 361, L45
Henkel, C., Mauersberger, R., \& Schilke, P. 1988, A\&A, 201, L23

Henkel, C., Mauersberger, R., Wiklind, T., Hüttemeister, S., Lemme, C., \& Millar, T. J. 1993, A\&A, 268, L17

Henkel, C., Tarchi, A., Menten, K. M., \& Peck, A. B. 2004, A\&A, 414, 117 Henkel, C., Whiteoak, J. B., \& Mauersberger, R. 1994, A\&A, 284, 17

Ho, P. T. P., Martin, R. N., Henkel, C., \& Turner, J. L. 1987, ApJ, 320, 663

Hollenbach, D. J., \& Tielens, A. G. G. M. 1997, ARA\&A, 35, 179

Hüttemeister, S., Henkel, C., Mauersberger, R., Brouillet, N. Wiklind, T., \& Millar, T. J. 1995, A\&A, 295, 571

Hüttemeister, S., Mauersberger, R., \& Henkel, C. 1997, A\&A, 326, 59

Israel, F. P., \& Baas, F, 2001, A\&A, 371, 433

Jacq, T., Baudry, A., Walmsley, C. M., \& Caselli, P. 1999, A\&A, 347, 957

Jansen, D. J., Spaans, M., Hogerheijde, M. R., \& van Dishoeck, E. F. 1995, A\&A, 303, 541

Johansson, L. E. B. 1991, in IAU Symp. 146, Dynamics of Galaxies and Their Molecular Cloud Distributions, ed. F. Combes \& F. Casoli (Dordrecht: Kluwer), 1

Kaifu, N., et al. 2004, PASJ, 56, 69

Kerr, F. J., \& Lynden-Bell, D. 1986, MNRAS, 221, 1023

Lee, C. W., Cho, S-H., \& Lee, S-M. 2001, ApJ, 551, 333

Lovas, F. J. 1992, J. Phys. Chem. Ref. Data, 21, 181 . 2004, J. Phys. Chem. Ref. Data, 33, 177

Magain, P., \& Gillet, D. 1987, A\&A, 184, L5

Martin, R. N., \& Ho, P. T. P. 1979, A\&A, 74, L7

Martín, S., Martín-Pintado, J., Mauersberger, R., Henkel, C., \& García-Burillo, S. 2005, ApJ, 620, 210

Martín, S., Mauersberger, R., Martín-Pintado, J., García-Burillo, S., \& Henkel, C. 2003, A\&A, 411, L465

Martín-Pintado, J., Bachiller, R., \& Fuente, A. 1992, A\&A, 254, 315

Martín-Pintado, J., de Vicente, P., Fuente, A., \& Planesas, P. 1997, ApJ, 482, L45

Martín-Pintado, J., Gaume, R., Rodríguez-Fernández, N. J., de Vicente, P., \& Wilson, T. L. 1999, ApJ, 519, 667

Martín-Pintado, J., Rizzo, J. R., de Vicente, P., Rodríguez-Fernández, N. J., \& Fuente, A. 2001, ApJ, 548, L65

Mattila, S., \& Meikle, W. P. S. 2001, MNRAS, 324, 325

Mauersberger, R., \& Henkel, C. 1989, A\&A, 223, 79

. 1991, A\&A, 245, 457 1993, Rev. Mod. Astron., 6, 69

Mauersberger, R., Henkel, C., \& Chin, Y.-N. 1995, A\&A, 294, 23

Mauersberger, R., Henkel, C., \& Sage, L. J. 1990, A\&A, 236, 63

Mauersberger, R., Henkel, C., Walmsley, C. M., Sage, L. J., \& Wiklind, T. 1991, A\&A, 247, 307

Mauersberger, R., Henkel, C., Weiss, A., Peck, A. B., \& Hagiwara, Y. 2003, A\&A, 403, 561

Mauersberger, R., Henkel, C., Wielebinski, R., Wiklind, T., \& Reuter, H.-P. 1996, A\&A, 305, 421

McGonagle, D., Irvine, W. M., \& Ohishi, M. 1994, ApJ, 422, 621

Meier, D. S., \& Turner, J. L. 2005, ApJ, 618, 259

Millar, T. J. 2002, Planet. Space Sci., 50, 1189

Minh, Y. C., Ziurys, L. M., Irvine, W. M., \& McGonagle, D. 1991, ApJ, 366, 192

Mouhcine, M., Ferguson, H. C., Rich, R. M, Brown, T. M., \& Smith, T. E. 2005, ApJ, 633, 810 
Nguyen-Q-Rieu, Henkel, C., Jackson, J. M., \& Mauersberger, R. 1991, A\&A, 241, L33

Nguyen-Q-Rieu, Jackson, J. M., Henkel, C., Truong-Bach, \& Mauersberger, R. 1992, ApJ, 399, 521

Nguyen-Q-Rieu, Nakai, N., \& Jackson, J. M. 1989, A\&A, 220, 57

Nummelin, A., Bergman, P., Hjalmarson, Å., Friberg, P., Irvine, W. M., Millar, T. J., Ohishi, M., \& Saito, S. 1998, ApJS, 117, 427 2000, ApJS, 128, 213

Ohishi, M., Irvine, W. M., \& Kaifu, N. 1992, in IAU Symp. 150, Astrochemistry of Cosmic Phenomena, ed. P. D. Singh (Dordrecht: Kluwer), 171

Ott, J., Weiss, A., Henkel, C., \& Walter, F. 2005, ApJ, 629, 767

Paglione, T. A. D., Tosaki, T., \& Jackson, J. M. 1995, ApJ, 454, L117

Paglione, T. A. D., Yam, O., Tosaki, T., \& Jackson, J. M. 2004, ApJ, 611, 835

Pence, W. D. 1981, ApJ, 247, 473

Peng, R., Zhou, S., Whiteoak, J. B., Lo, K. Y., \& Sutton, E. C. 1996, ApJ, 470, 821

Petuchowski, S. J., \& Bennett, C. L. 1992, ApJ, 391, 137

Pety, J., Teyssier, D., Fosse, D., Gerin, M., Roueff, E., Abergel, A., Habart, E., \& Cernicharo, J. 2005, A\&A, 435, 885

Pickett, H. M., et al. 1998, J. Quant. Spectrosc. Radiat. Transfer, 60, 883

Pineau des Forêts, G., Roueff, E., \& Flower, D. R. 1989, MNRAS, 240, 167

Puche, D., Carignan, C., \& van Gorkom, J. H. 1991, AJ, 101, 456

Puxley, P. J., Mountain, C. M., Brand, P. W. J. L., Moore, T. J. T., \& Nakai, N. 1997, ApJ, 485, 143

Requena-Torres, M. A., Martín-Pintado, J., Rodríguez-Franco, A., Martín, S., Rodríguez-Fernández, N. J., \& de Vicente, P. 2006, A\&A, in press (astro-ph/ 0605031)

Rickard, L. J., Palmer, P., Morris, M., Zuckerman, B., \& Turner, B. E. 1975, ApJ, 199, L75

Rickard, L. J., Palmer, P., Turner, B. E., Morris, M., \& Zuckerman, B. 1977, ApJ, 214, 390

Rieke, G. H., Lebofsky, M. J., Thompson, R. I., Low, F. J., \& Tokunaga, A. T. 1980, ApJ, 238, 24

Rodríguez-Fernández, N. J., \& Martín-Pintado, J. 2005, A\&A, 429, 923

Rodríguez-Fernández, N. J., Martín-Pintado, J., Fuente, A., \& Wilson, T. L. 2004, A\&A, 427, 217

Sage, L. J., Henkel, C., \& Mauersberger, R. 1991, A\&A, 249, 31
Sage, L. J., \& Ziurys, L. M. 1995, ApJ, 447, 625

Sandage, A. R., \& Tammann, G. A. 1987, A Revised Shapley-Ames Catalog of Bright Galaxies (2nd ed.; Washington: Carnegie Inst.)

Sato, F., Hasegawa, T., Whiteoak, J. B., \& Miyawaki, R. 2000, ApJ, 535, 857

Schilke, P., Pineau des Forêts, G., Walmsley, C. M., \& Martín-Pintado, J. 2001, A\&A, 372, 291

Scoville, N. Z., Soifer, B. T., Neugebauer, G., Matthews, K., Young, J. S., \& Yerka, J. 1985, ApJ, 289, 129

Scoville, N. Z., Solomon, P. M., \& Penzias, A. A. 1975, ApJ, 201, 352

Seaquist, E. R., \& Bell, M. B. 1986, ApJ, 303, L67

Sofue, Y. 1990, PASJ, 42, 827

Soifer, B. T., Boehmer, L., Neugebauer, G., \& Sanders, D. B. 1989, AJ, 98, 766 Solomon, P. M., \& de Zafra, R. 1975, ApJ, 199, L79

Stark, A. A., \& Wolff, R. S. 1979, ApJ, 229, 118

Strickland, D. K., Heckman, T. M., Colbert, E. J. M., Hoopes, C. G., \& Weaver, K. A. 2004, ApJ, 606, 829

Takano, S., Nakai, N., \& Kawaguchi, K. 1995, PASJ, 47, 801 2002, PASJ, 54, 195

Thompson, R. I., Lebovski, M. J., \& Rieke, G. H. 1978, ApJ, 222, L49

Turner, B. E. 1985, ApJ, 299, 312 1989, ApJS, 70, 539 1991, ApJS, 76, 617

Ulvestad, J. S., \& Antonucci, R. R. J. 1997, ApJ, 488, 621

Usero, A., García-Burillo, S., Fuente, A., Martín-Pintado, J., \& RodríguezFernández, N. J. 2004, A\&A, 419, 897

Vidal-Madjar, A. 2002, Planet. Space Sci., 50, 1161

Wang, M., Henkel, C., Chin, Y.-N., Whiteoak, J., Cunningham, M., Mauersberger, R., \& Muders, D. 2004, A\&A, 422, 883

Weiss, A., Neininger, N., Henkel, C., Stutzki, J., \& Klein, U. 2001, ApJ, 554, L143

Weliachew, L. 1971, ApJ, 167, L47

Whiteoak, J. B., Gardner, F. F., \& Höglund, B. 1980, MNRAS, 190, 17

Wild, W. 1990, Ph.D. thesis, Ludwig-Maximilian-Univ., München

Wyrowski, F., Schilke, P., \& Walmsley, C. M. 1999, A\&A, 341, 882

Zinchenko, I., Henkel, C., \& Mao, R. Q. 2000, A\&A, 361, 1079

Ziurys, L. M., Friberg, P., \& Irvine, W. M. 1989, ApJ, 343, 201 